\title{
APPENDIX I. BATHYMETRIC, MAGNETIC AND SEISMIC REFLECTION DATA, DEEP SEA DRILLING PROJECT, LEG 25
}

\author{
R. Schlich, Institut de Physique du Globe de Paris, St. Maur-des-Fossés, France
}

The Glomar Challenger, DSDP Leg 25, started from Port Louis (Mauritius) on 28 June 1972 and ended in Durban (South Africa) on 22 August 1972 after having occupied 11 sites and steamed 5408 nautical miles in the western Indian Ocean. The underway data (bathymetry, magnetics, and seismic reflection profiles) collected during the cruise are presented as two separate sets of profiles. The location of these profiles is given on the track chart of Figure 1, which has been derived from all available navigational data given in Tables 1 to 16.

The first set of profiles is a computer plot of depth and total intensity magnetic anomalies presented as a function of distance, location, and time. The same distance and time scale is used for both the bathymetry and the magnetics in order to allow easy comparisons and possible correlations (Figure 2). The data are presented in several sections, which take into account either major course changes or drill site locations. Each section is identified by a code number: GC25-1 corresponds to the first section, from Mauritius to Site 239, and GC25-2 to the second section, from Site 239 to position $16^{\circ} 55.1^{\prime} \mathrm{S}$ and $54^{\circ} 21.4^{\prime} \mathrm{E}$ where the ship's track is altered from a north northeast course to north, northwest course, etc. These code numbers appear on each section at the bottom left corner of the figure and also along the corresponding track in Figure 1. The data collected in the vicinity of a drill site, which belong more appropriately to the site survey, are not given in this chapter. The bottom profiles indicate the depth in meters, assuming a sound velocity for seawater of $1500 \mathrm{~m} / \mathrm{sec}$; the scale is mentioned at the start of each section. The vertical exaggeration of the depth profile is $37: 1$. The top profiles represent the total intensity magnetic anomaly in gammas with the scale also given at the beginning of each section. The regional magnetic field has been computed and removed using the Cain et al. (1964) POGO 10/68 coefficients. All fixes and "pit log" positions that were used (see Tables 1 to 16) are marked on each section by a vertical line of dots; selected positions, depending on space possibilities as allotted by the computer printout, are given in full on the diagram, and the dots are aligned with the lower part of those numbers which correspond to that position. The vertical spacing of these dots corresponds, for the depth profiles, to a 1000-meter interval and, for the magnetics, to a 200 -gamma anomaly amplitude. The crosses on these vertical lines indicate sea level for the depth profile and the zero level for the magnetic anomaly profile. GMT time in hours is marked and labelled every 6 hours at the top of each figure. The dates (day and month) also are shown at the same level of the diagram directly after 0000 GMT of each day. A distance scale, annotated every 100 nautical miles, is given at the bottom of each figure; all the sections start at zero mile and the last figure along this scale corresponds to the section's total length in nautical miles. The courses (true course) and large course changes, if allowed by space requirements, are given just above the distance scale at the 6000-meter depth line.

The second set of data are photographic reductions of the original 10-sec-sweep seismic reflection profiles and, consequently, are displayed as a function of time only (Figure 3). The section code numbers (GC25-1, GC25-2, etc.), used for the bathymetric and magnetic profiles, also appear on the photographs. GMT time in hours is marked and labeled every 4 hours at the bottom of each photograph. The mean course for each section is given in brackets after each section code number; speed information is shown at the beginning of each photograph just above the hour scale and whenever large changes (about $1 \mathrm{kn}$ or more) occur for at least one hour of time. Significant recording interruptions are indicated by two hash marks. Vertical scales are in seconds of acoustic two-way travel time (DT). The vertical exaggeration varies, according to the speed, and is between 15:1 and 30:1. Site approaches and site surveys usually are done at 5 or 7 knots, while underway data, collected between sites, usually are obtained at 8 to 11 knots. For the convenience of those who will use this information, the seismic reflection profiles have been reversed and can be read from left to right on each sheet. Comparison with the first set of data (bathymetry and magnetics) is thus much easier. The site survey data are not presented in this chapter; they appear in the site reports (Chapters 2-10, this volume).

The navigational data for Glomar Challenger DSDP Leg 25 , are summarized in Tables 1 to 16, where all fixes and all "pit log" positions are listed. Each table corresponds to one section. The first "distance" column (DIST) gives the distance in nautical miles between two successive positions; the corresponding true course and effective speed are given in the two next columns (CSE, SPEED). The last column on the right (DISTT) indicates the total distance from the start of each section to the position which is considered; the last figure at the bottom of that column represents the total length of the corresponding section. The site survey navigation data are not presented in these tables. The different sections which have been distinguished are indicated on the track chart of Figure 1. Along this track, GMT hour marks have been drawn every 3 hours and labeled every 12 hours. The site survey tracks are hardly distinguishable on this chart; they are given with more details in the respective site reports (see Chapters 2-10, this volume).

The bathymetric data were obtained with a Gifft precision depth recorder using a $12-\mathrm{kHz}$ transducer-receiver system. The magnetic data were collected with a Varian proton-precession magnetometer with the sensor towed 300 
meters behind the ship. The readings on the recorder were made every 5 minutes. The seismic reflection profiler consisted of two Bolt PAR 600 A airguns, a towed hydrophone array assembled by DSDP, a Bolt amplifier and bandpass filter, and an EDO Western Model PDR 333 recorder. Navigation was performed using an ITT Model satellite receiver and a PDP 8 computer system. Smooth navigation plots, bathymetric and magnetic plots were made using Institut National d'Astronomie et de Géophysique de Paris, IBM 360-65 computer.

Glomar Challenger left Port Louis (Mauritius) in a westerly direction and reached Site 239 after 1.5 days of steaming across the northern part of the Madagascar Basin and the southern part of the Mascarene Basin. Reunion Island was passed to the north; somewhat later, on 29 June 1972 at about 1700 GMT, an important fracture zone, unofficially named Mahanoro Ridge (Conrad 14 cruise) and running in a north northeast-south southwest $\left(\sim 35^{\circ}\right)$ direction to the southern tip of Madagascar, was crossed. The basement reflector appeared clearly on the airgun record after having crossed this structure, and the selection of Site 239 was thus very easy.

Site 239 was left in a north northeast direction. The purpose of this slight deviation from the direct track to Site 240 was to attempt the recognition of possible sea floor spreading magnetic anomalies in the Mascarene Basin since no account of magnetic identification was available for this area. Some lineations (sections GC25-1 and GC25-2) were indeed observed, but the profiles were too short to be able to propose any identification. On 4 July 1972, at about $17^{\circ} 40^{\prime}$ S (between 1500 and 2000 GMT), the ship's track crossed another important fracture zone which, according to the general bathymetric trend in this area, seems to run parallel to the so-called Mahanoro Ridge. At position $16^{\circ} 55^{\prime} \mathrm{S}$ and $54^{\circ} 21^{\prime} \mathrm{E}$, the course was changed to a north northwest direction. Glomar Challenger passed west of Tromelin Island, then along the Amirante Trench through the Farquhar group. Extremely rough basement topography, associated with large magnetic anomalies ranging between 200 and 800 gammas, was observed all along this track (section GC25-3). Entering in the southwestern Somali Basin, basement reflections could no longer be resolved. However, it was known from previous work (Bunce et al., 1967; Schlich et al., 1972) that the sediment cover was relatively thin, 1.0 seconds to 0.5 seconds DT (double way time) and even less over small topographic highs. Near site 240 , the airgun records showed a thin transparent sediment layer overlying a stratified sequence of poorly defined thickness, which prevented any precise basement depth computation.

From Site 240, Glomar Challenger proceeded west to Site 241, which is located on the lower East African continental rise. At about 1100 GMT on 12 July 1972, the ship's track (section GC25-4) crossed a very distinct topographic high associated with a 300 -gamma magnetic anomaly. This feature may be interpreted as the southern continuation of Chain Ridge. Farther to the west, the sediment thickness increases very rapidly, and it has been shown (Schlich et al., 1972) that basement is at least as deep as 4000 meters below the sea floor at Site 241 .
On leaving Site 241, Glomar Challenger had to change course twice for technical reasons (sections GC25-5 and GC25-6) and then proceeded south towards Site 242 (section GC25-7). The sea floor rises gently from 4000 meters to about 2500 meters and is cut by several submarine canyons, which are clearly shown on the airgun records. The sediments remain very thick and basement reflections cannot be seen. At latitude $15^{\circ} 55.7^{\prime} \mathrm{S}$, about 20 miles due west of the proposed Site 242, Glomar Challenger changed course to about $090^{\circ}$ and crossed the so-called Davie Ridge at a right angle. No significant magnetic anomaly was recorded across the ridge (less than 50 gammas). Acoustic basement appears clearly on the airgun record but does not correspond to real basement (as proven later by Marion Dufresne, Cruise 2, 1973).

Proceeding from this point south towards Sites 243 and 244 , only small magnetic anomalies were recorded; they are associated with topographic highs along the north-south trending Davie Ridge and appear as short wavelength anomalies, probably related to the presence of volcanic material (section GC25-8). Before reaching Sites 243/244, the Zambesi Canyon was crossed several times, but the acoustic basement traced into and across the area with a flexotir device (Gallieni, Cruise 6, 1972) could not be seen on the Glomar Challenger airgun record (Chapter 6, this volume).

The Glomar Challenger left Site 244 on 29 July 1972, passed the Zambesi Canyon again, and steamed across the Madagascar Ridge in a southeast direction towards Site 245. Very large magnetic anomalies associated with rugged topography were recorded along this track (section GC25-9). South of Cap Ste. Marie, Madagascar, these anomalies exceed 1000 gammas in amplitude. In the Madagascar Basin, basement is clearly observed on the airgun record and can be traced into, and across, the area of Site 245. Magnetic anomalies of 400 gammas amplitude were recorded. This is somewhat surprising considering the parallelism of Glomar Challenger track with the anticipated magnetic lineation trends in this region (Schlich, Patriat, and Segoufin, 1972). These anomalies are without doubt related to the successive basement highs which can be seen on the airgun records.

In order to drill an intermediate site, which was located in the extreme southern part of the Madagascar Basin, Site 245 was left in a southwesterly direction (section GC25-10). In fact, north of Site 245, basement topography becomes rapidly very rough; this may be explained by the proximity of the Southwest Indian Ridge. Moreover, although it has been established (Schlich, this volume) that Site 245 is located on anomaly 29 of the Heirtzler et al. (1968) time scale, older anomalies, such as anomaly 30 , could not be identified from the magnetic data recorded along this track. Because of these two facts, plus unfavorable weather conditions, this proposed intermediate site was abandoned and Glomar Challenger steamed west towards Sites 246 and 247, located on the Madagascar Ridge (sections GC25-11 and GC25-12). A series of well-stratified layers, following more or less the gentle upward slope of the east flank of the Madagascar Ridge, can be traced on the Glomar Challenger airgun record; but the 
acoustic basement, which corresponds to a high velocity layer $(5 \mathrm{~km} / \mathrm{sec})$ according to Gallieni Cruise 4 (1971), appears only faintly on these records. Magnetic anomalies of relatively long wavelengths $(\sim 80 \mathrm{~km})$ are observed in the lower part of the eastern flank of the ridge, and short wavelength anomalies of low amplitude on the ridge crest itself may indicate minor volcanic intrusives.

By contrast, the western flank of the Madagascar Ridge is very steep and suggestive of large-scale vertical faulting. A magnetic anomaly of more than 1000 gammas amplitude corresponds to this abrupt boundary (section GC25-13). In the Mozambique Basin, the basement was not recorded continuously, but its presence can be inferred at some locations. Patches of very transparent sediments overlie a sequence of well-stratified layers which thin towards the Mozambique Ridge (section GC25-14).

Before reaching Site 248, at about 0400 GMT on 13 August 1972, a clear intrusive structure that is associated with a large amplitude magnetic anomaly was observed on the records (section GC25-13). At Site 248, the basement reflector is distinct and the selection of the drill site was very easy.

The very steep eastern flank of the Mozambique Ridge was crossed enroute to Site 249. The airgun record (section GC25-15) shows a very rough basement topography overlain by sediment accumulations of variable thickness $(0$ to 1 second DT). The magnetic anomalies over the ridge have relatively long wavelengths $(\sim 50 \mathrm{~km})$ and have an amplitude range of about 400 gammas. Site 249 was left on 20 August 1972, and Glomar Challenger steamed westward over the western flank of the Mozambique Ridge, which gradually descends into the Natal Valley, and finally called at Durban on 22 August 1972 (section GC25-16).

\section{ACKNOWLEDGMENTS}

The data were collected at sea by the shipboard technicians under the direction of T. Gustafson; their important contribution is gratefully acknowledged. The navigational data and the bathymetric and magnetic computer plots were processed by $\mathrm{M}$. Bitterly and $\mathrm{M}$. Scaglia at Institut de Physique du Globe de Paris, Saint-Maur-des-Fossés, France. The seismic reflection profiles were prepared by DSDP technical staff whose help also is greatly appreciated.

I wish also to thank the Global Marine shipboard staff, in particular, Captain J. Clarke and Operations Manager J. Shore.

\section{REFERENCES}

Bunce, E. T., Langseth, M. G., Chase, R. L., and Ewing, M., 1967. Structure of the western Somali Basin: J. Geophys. Res., v. 72, no. 10, p. 2547-2555.

Cain, J. C., Hendricks, S., Daniels, W. E., and Jensen, D. C., 1964. Computation of the main geomagnetic field from spherical expansion: NASA X 611-64-316.

Heirtzler, J. R., Dickson, G. O., Herron, E. M., Pittman, W. C., III, and LePichon, X., 1968. Marine magnetic anomalies, geomagnetic field reversals and motions of the ocean floor and continents: J. Geophys. Res., v. 73, no. 6, p. 2119-2136.

Schlich, R., Aubertin, F., Delteil, J. R., Leclaire, L., Magnier, P., Montadert, L., Patriat, P., and Valery, P., 1972. Donneés nouvelles sur le substratum du Bassin de Somalie à partir d'un profil de sismique reflexion: C. R. Acad. Sci. Paris, v. 275, p. 1331-1334.

Schlich, R., Patriat, P., and Segoufin, J., 1972. Compte Rendu d'activité pour la période du ler Avril 1971 au 29 Fevrier 1972: Note I. P. G. de Paris, no. 46. 


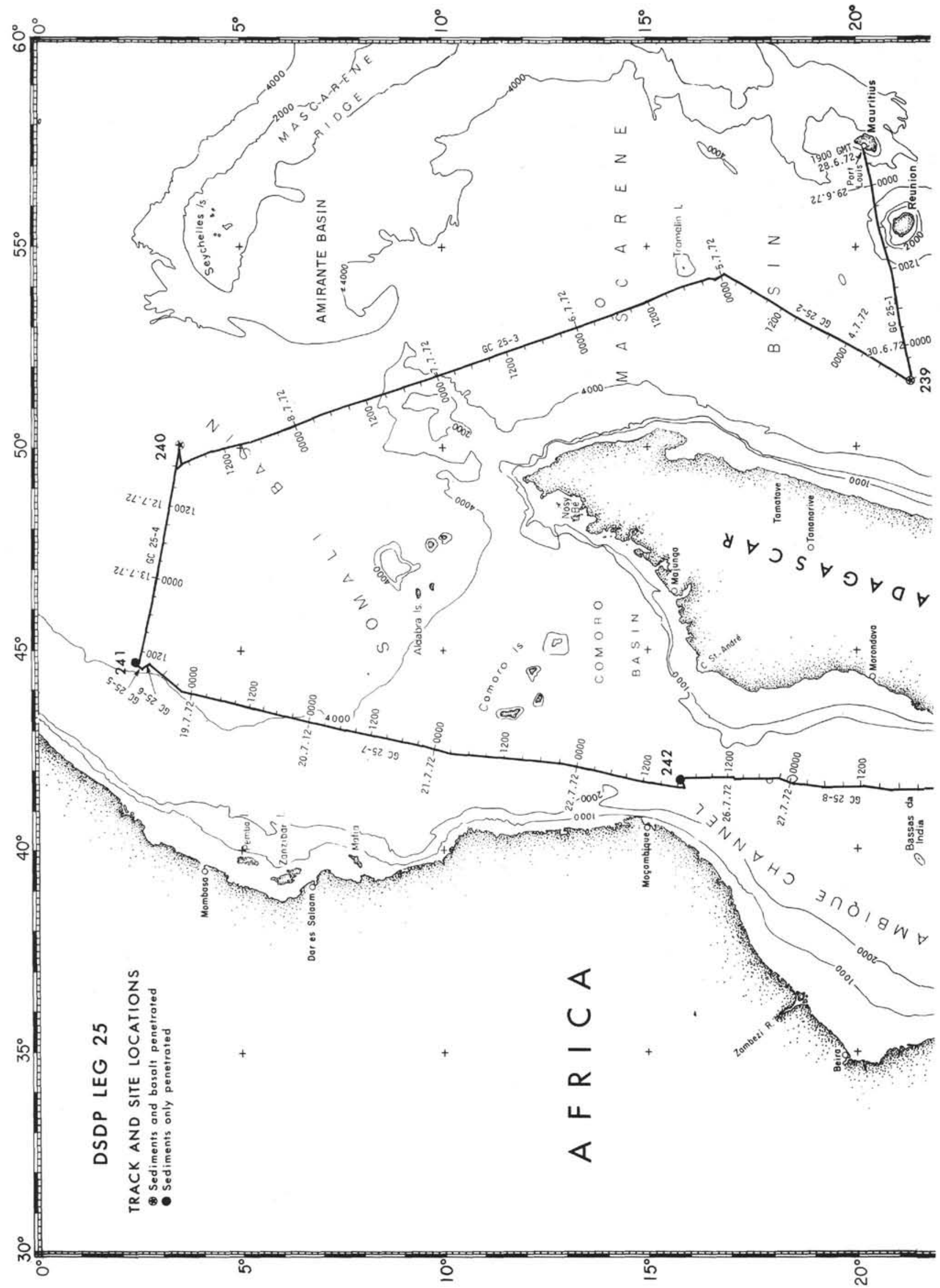

Figure 1. Glomar Challenger, DSDP Leg 25, Track Chart from Mauritius to Durban. 


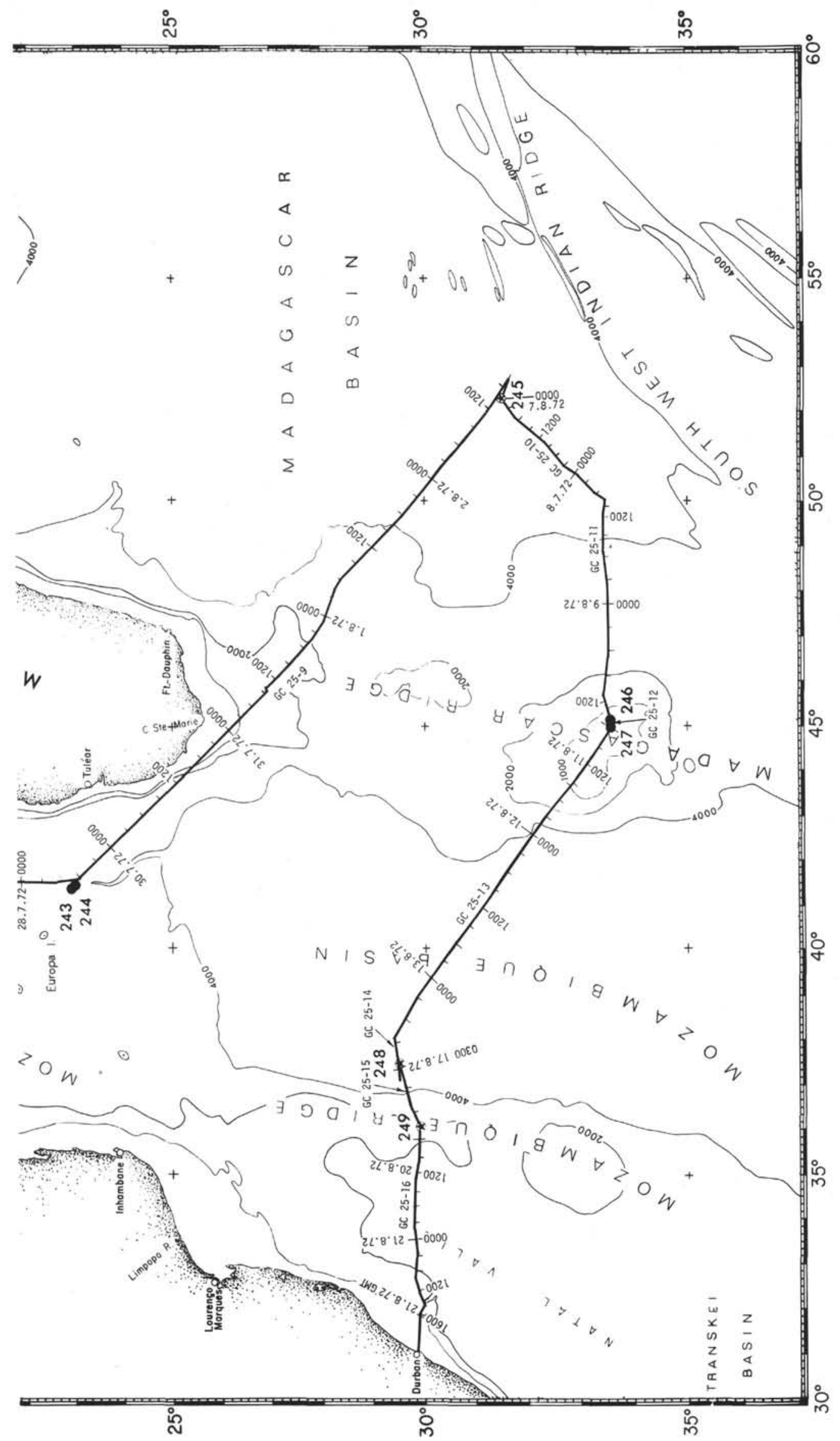




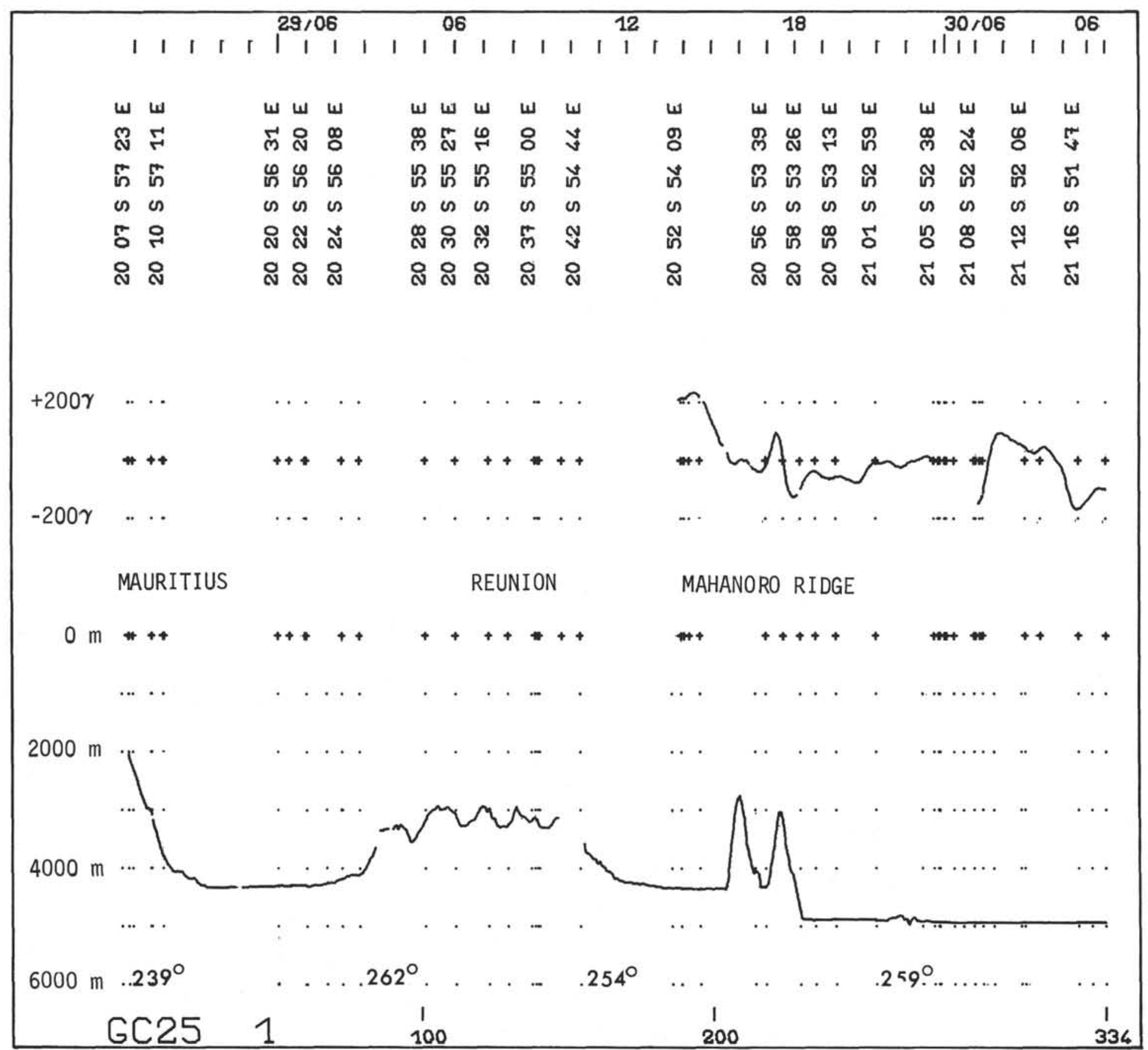

Figure 2. Glomar Challenger, DSDP Leg 25, depth and total intensity magnetic anomaly profiles displayed as a function of time, location, and distance. 


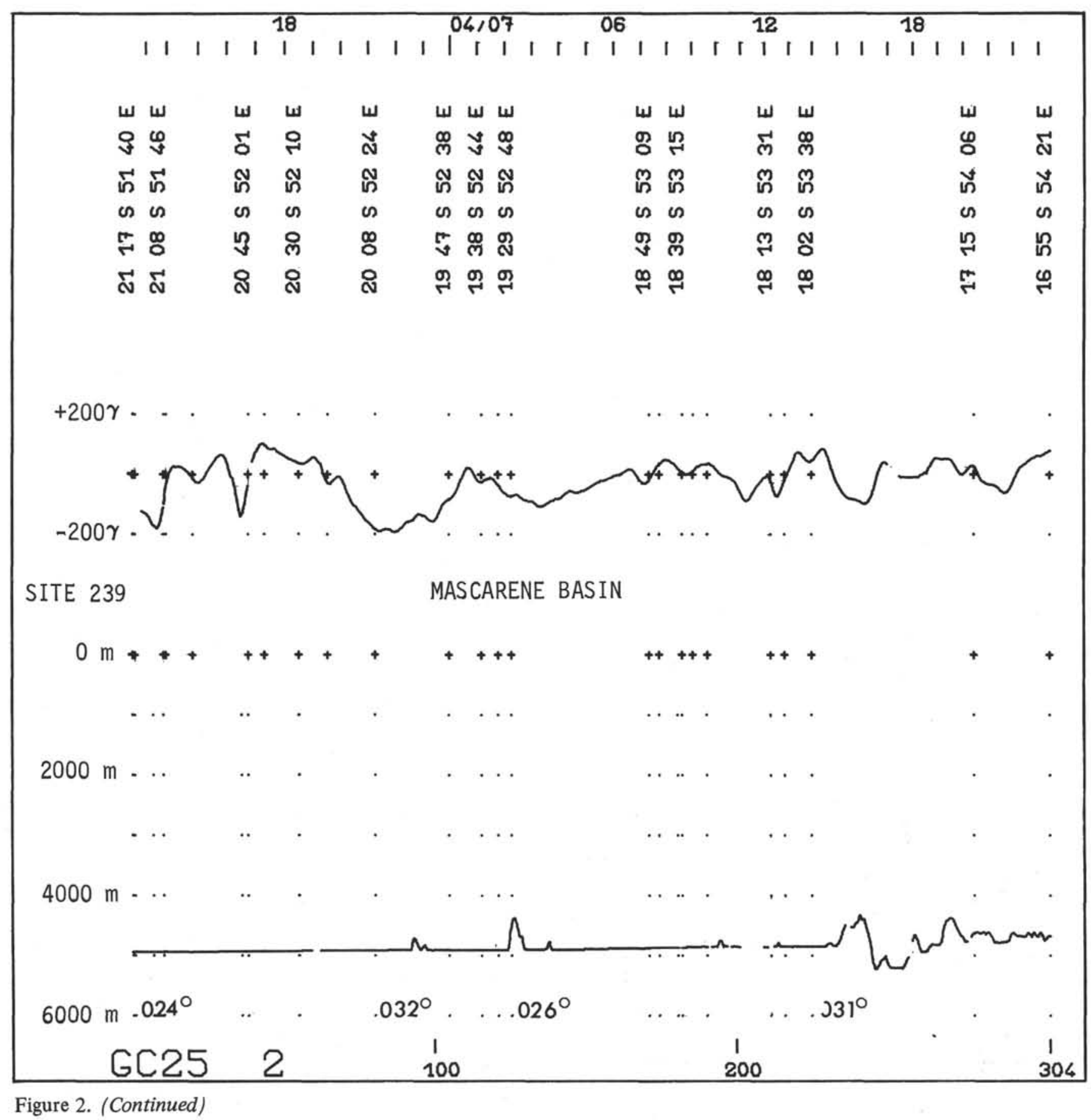




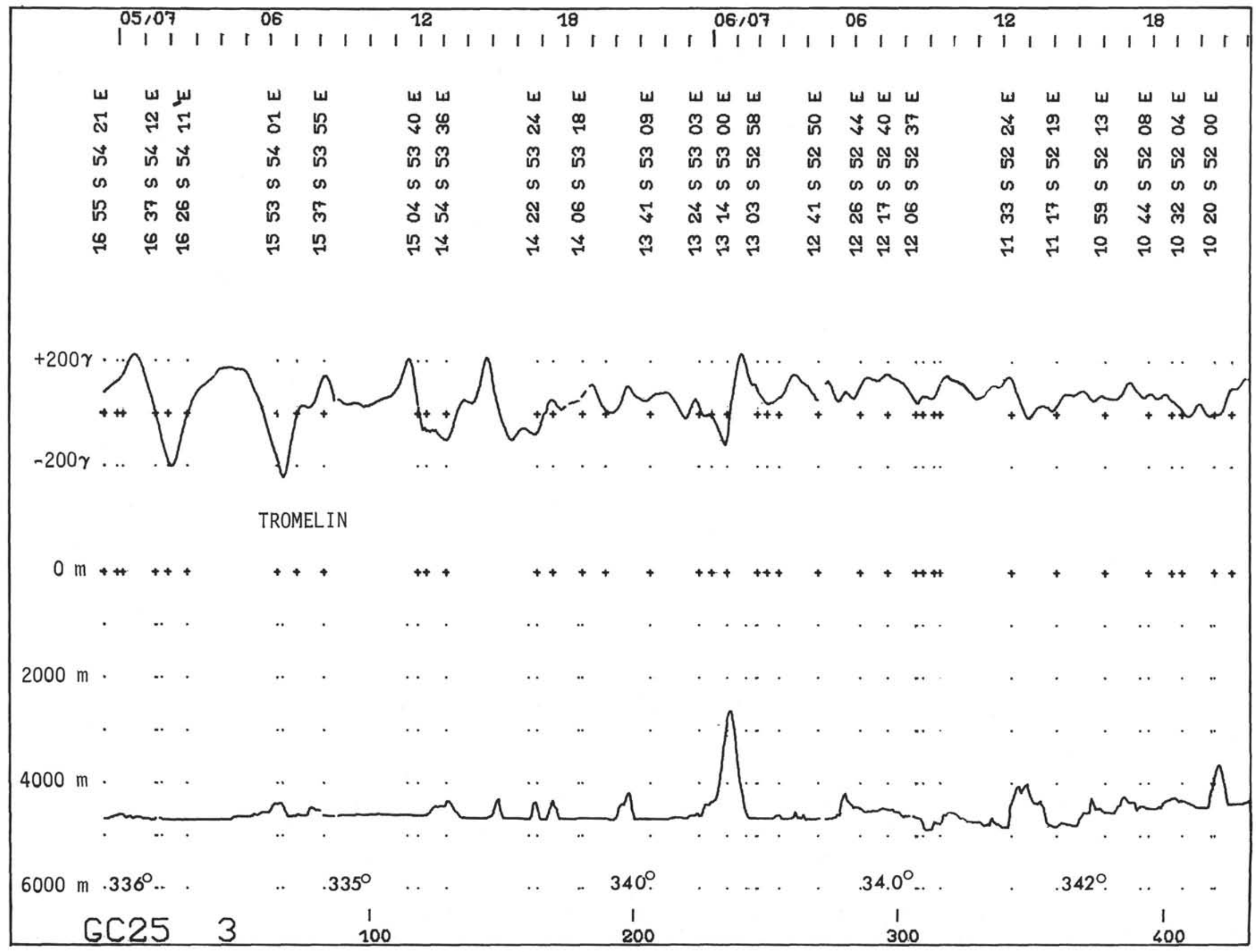




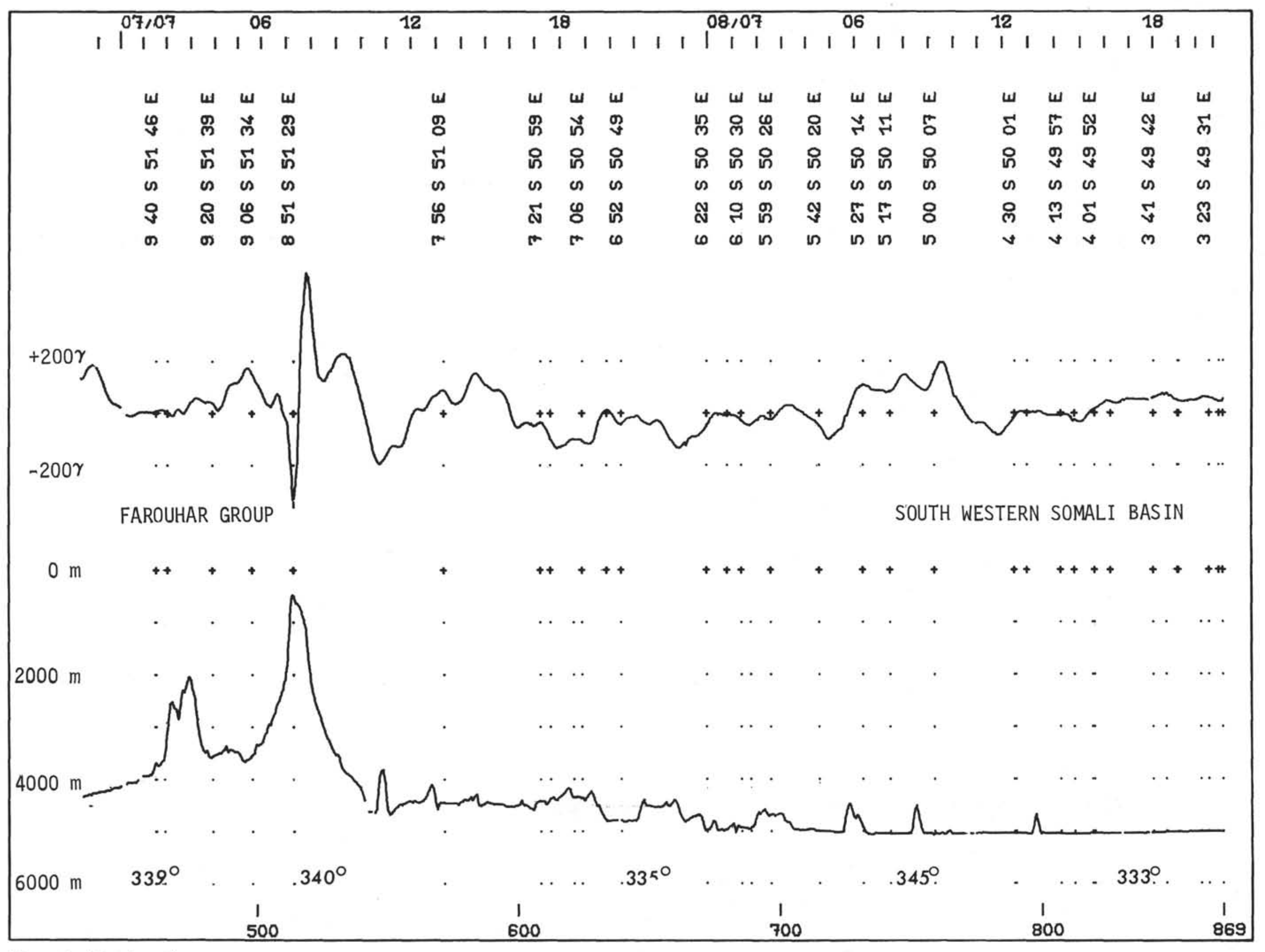

Figure 2. (Continued) 


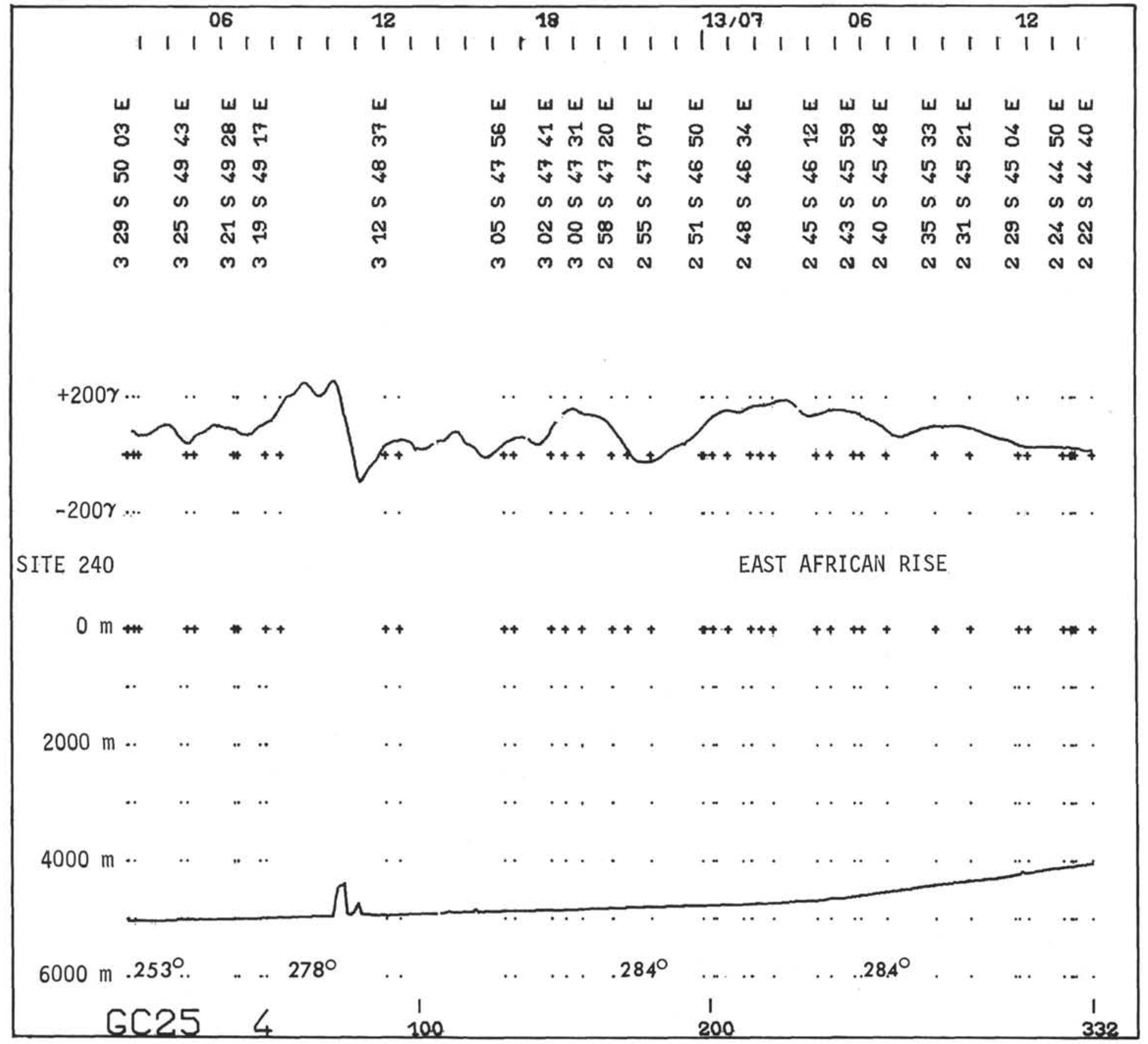

Figure 2. (Continued) 


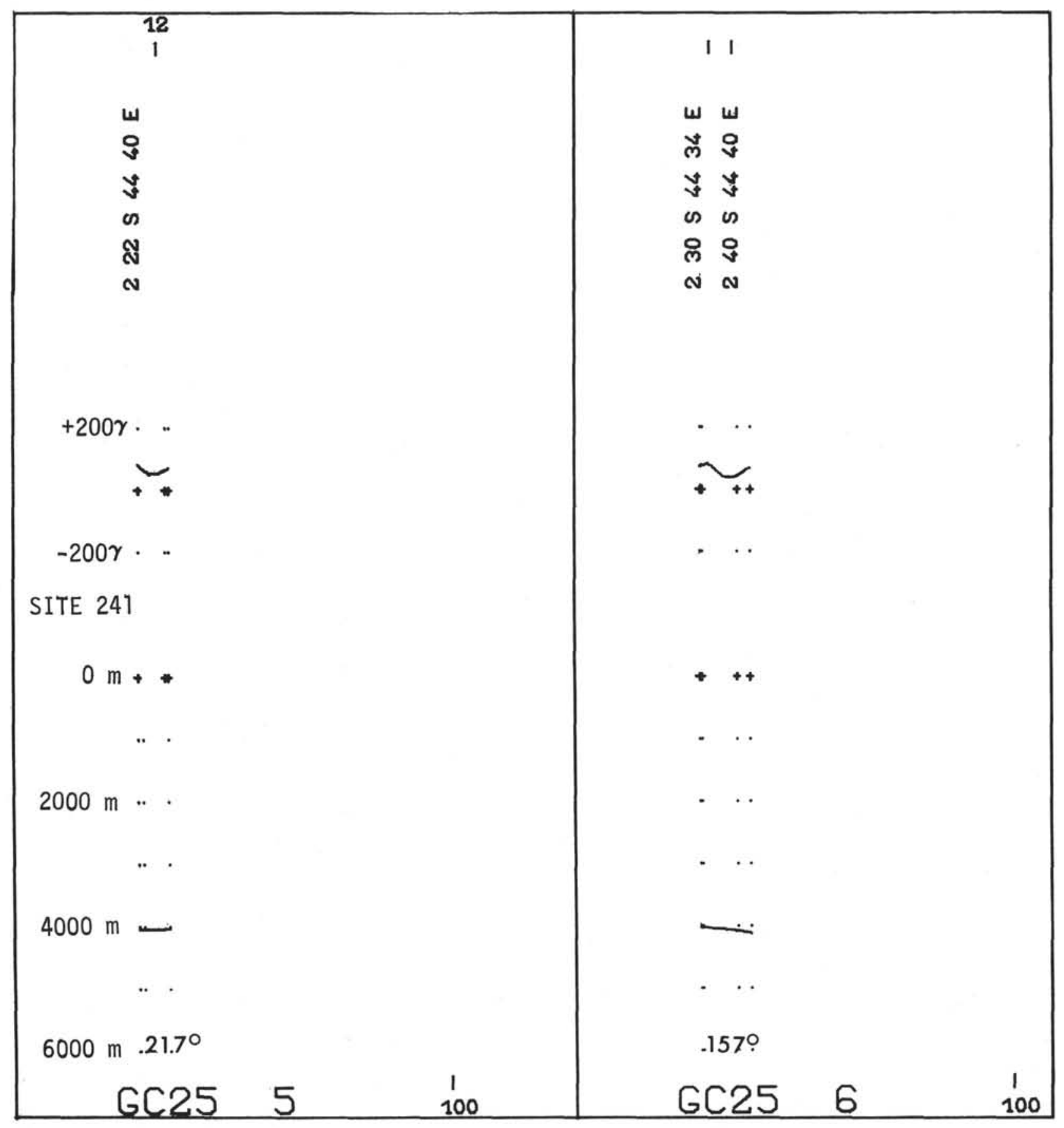




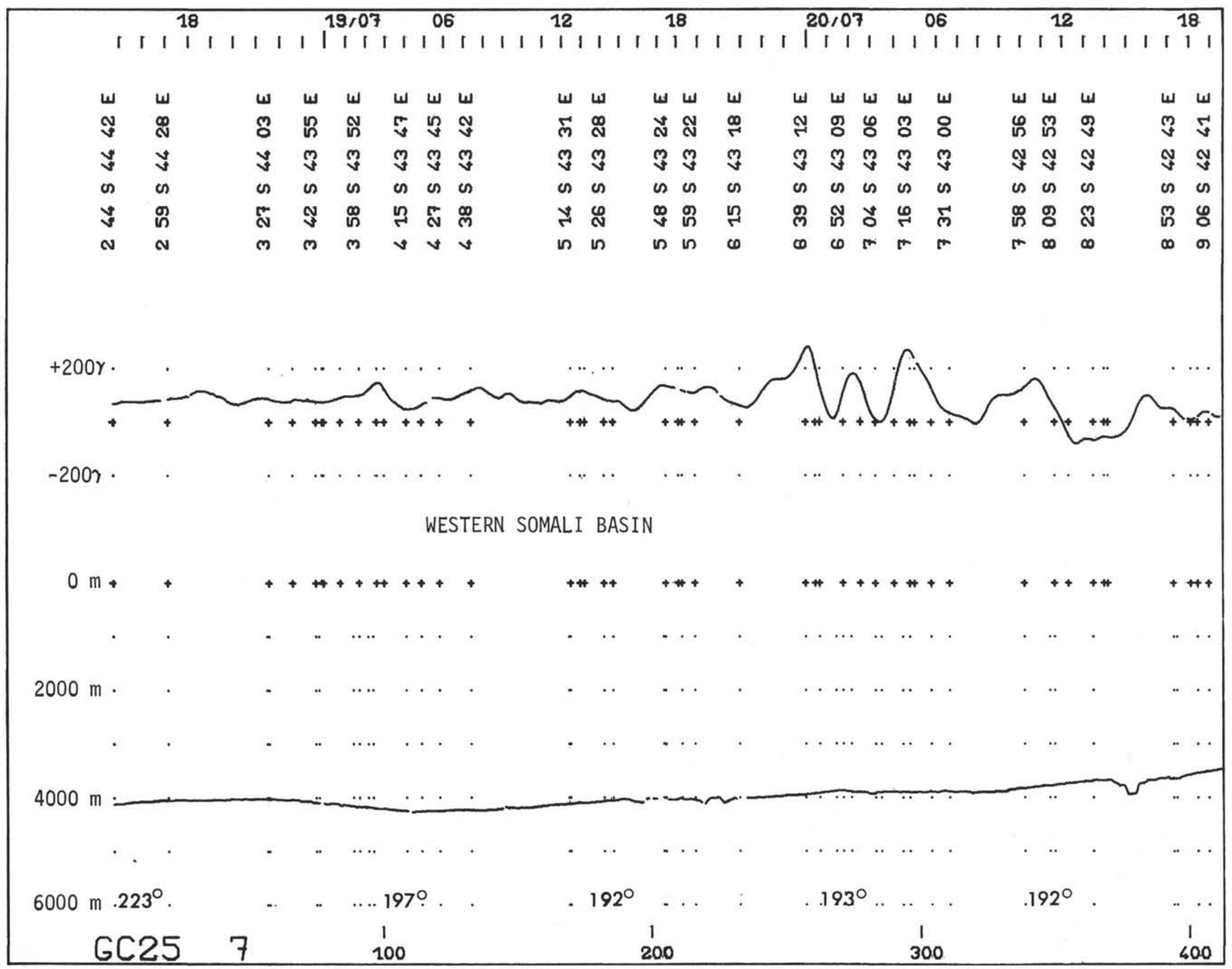




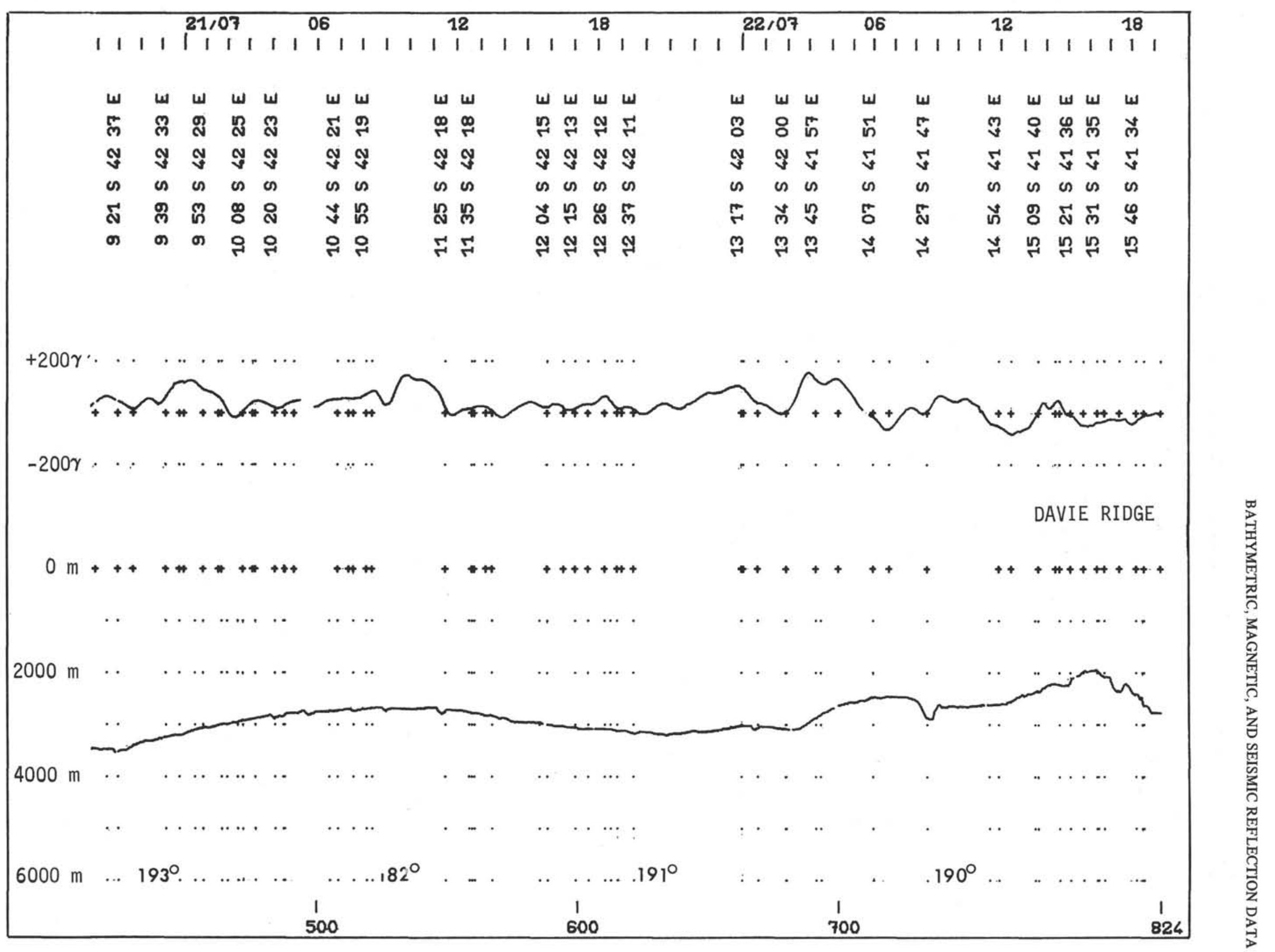

Figure 2. (Continued) 


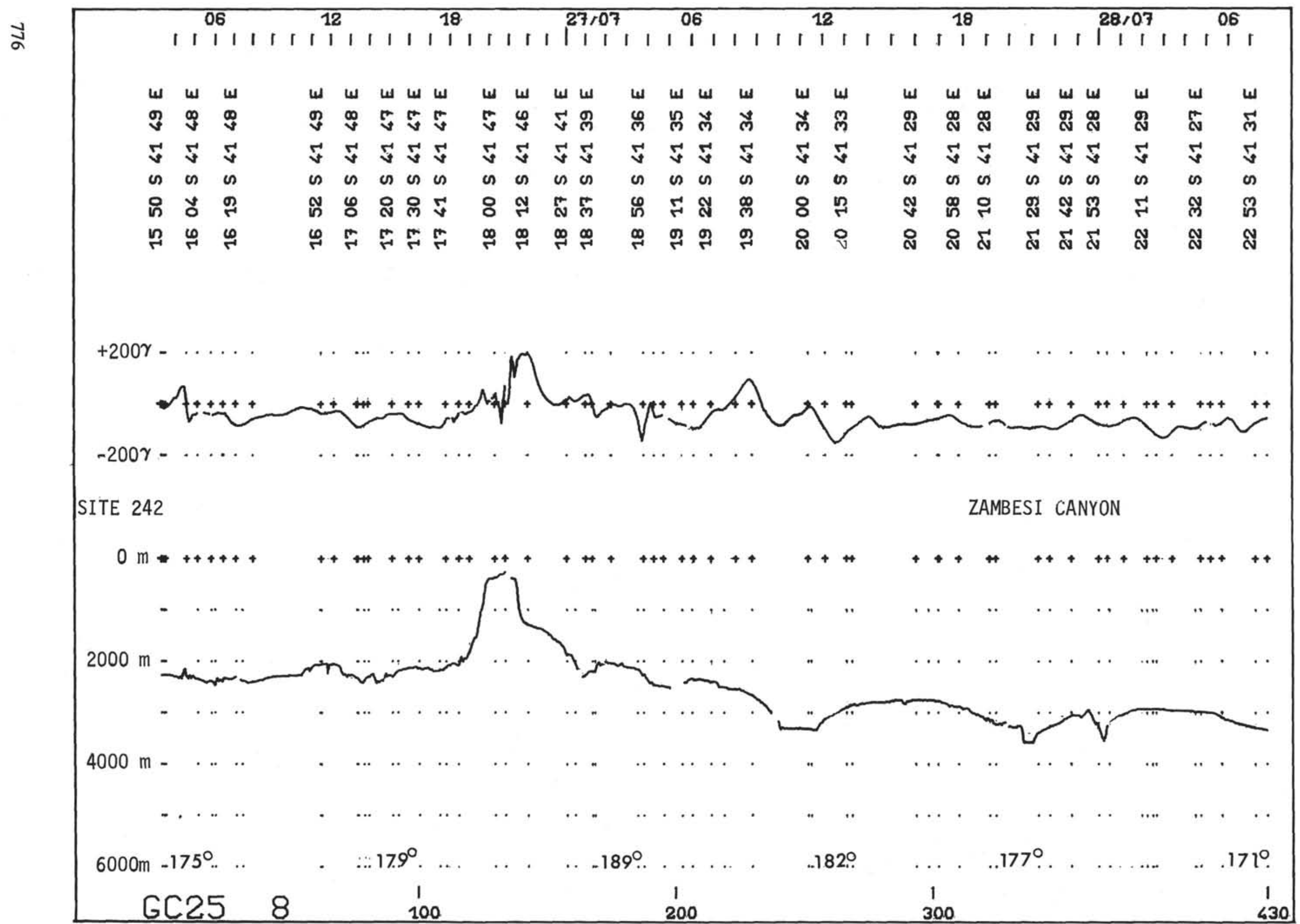

Figure 2. (Continued) 


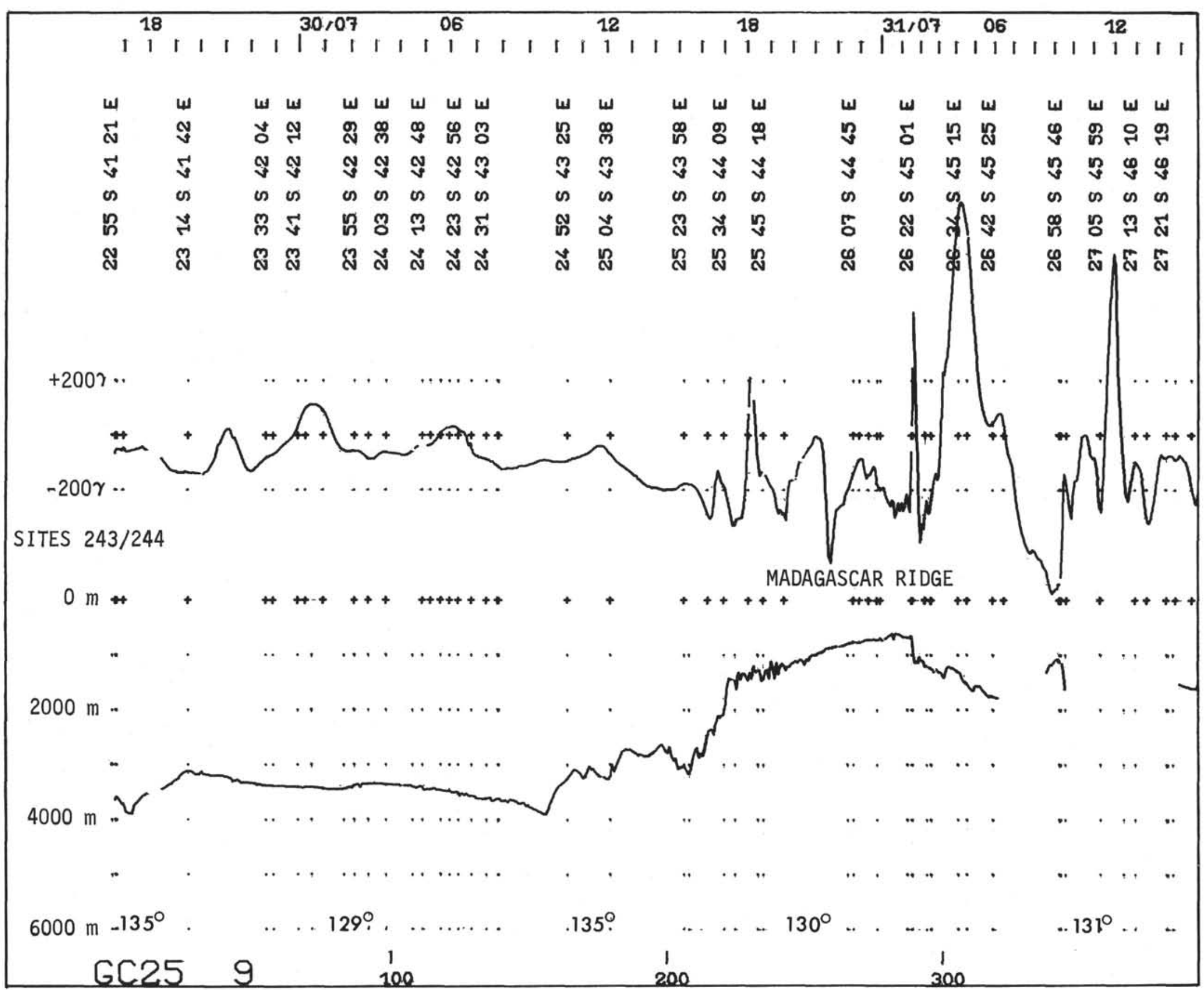




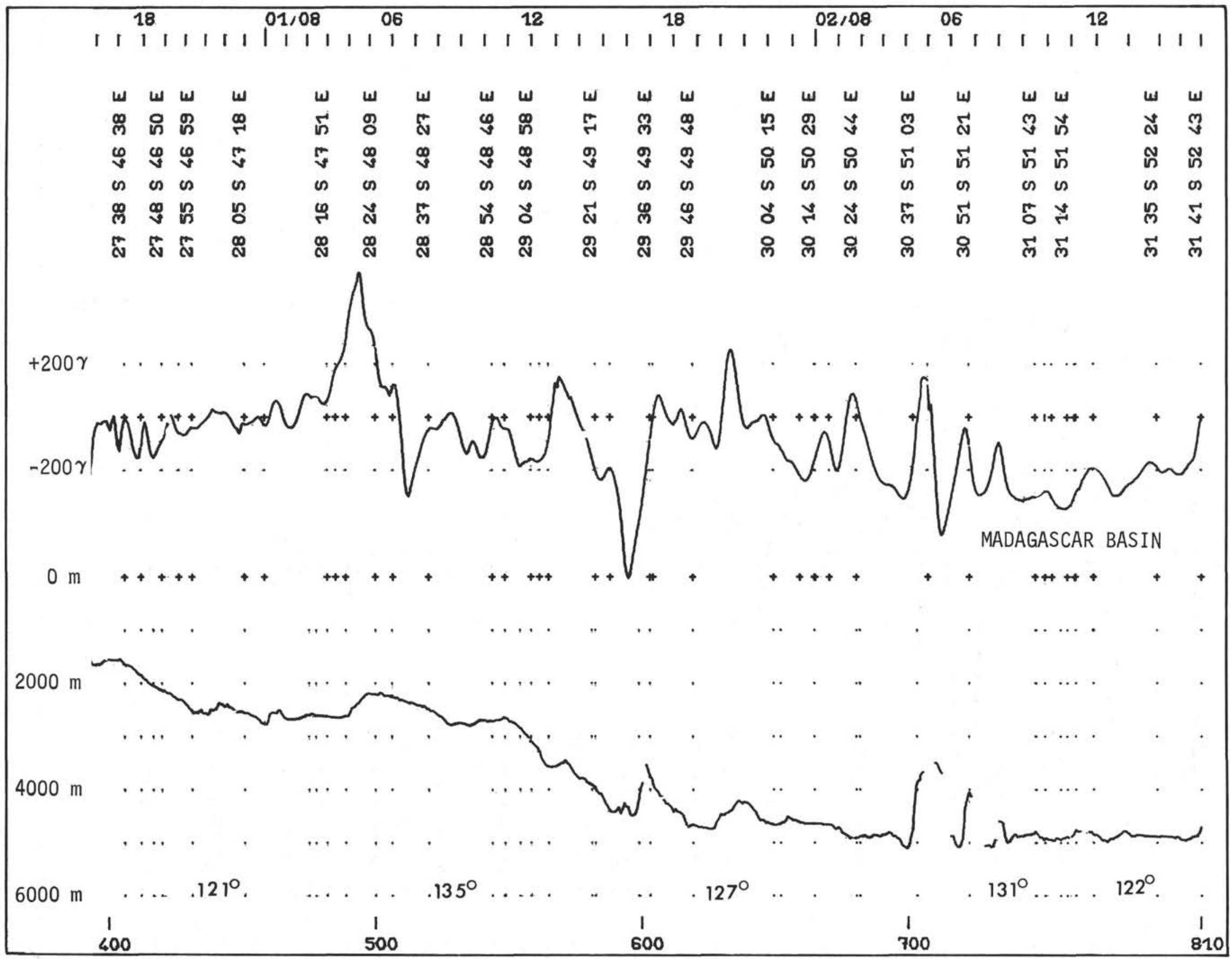




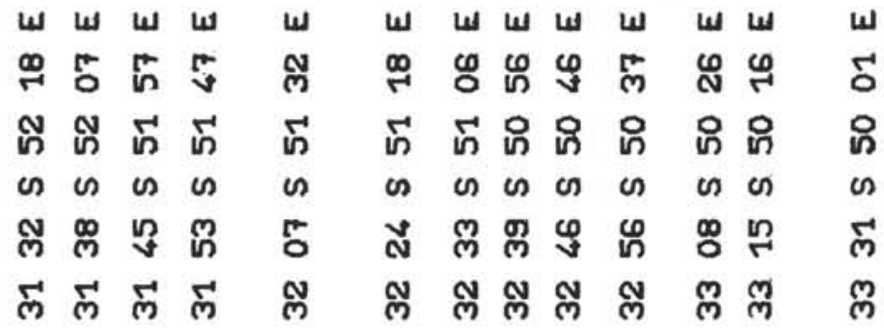

$+200$

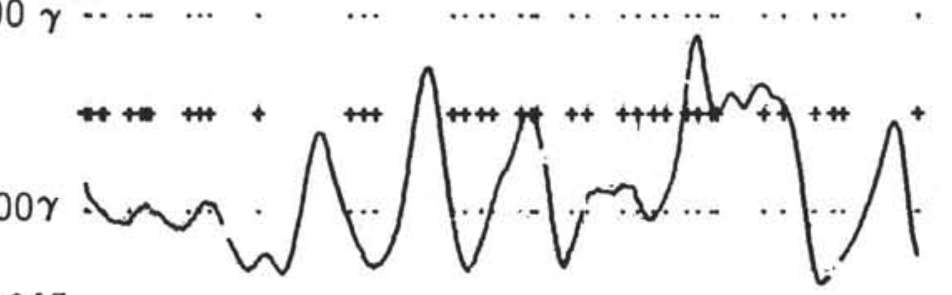

SITE 245

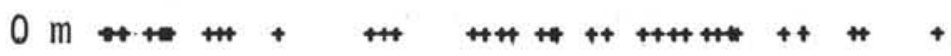

$2000 \mathrm{~m}$

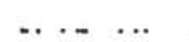

$4000 \mathrm{~m}$

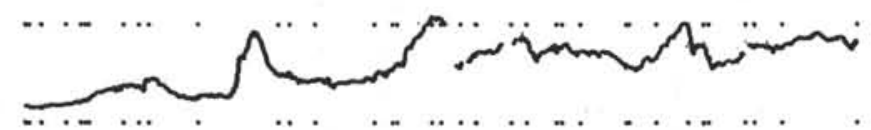

6000 m $25 z^{\circ} \ldots$

. . $237^{\circ}$. $.217^{\circ}$ 


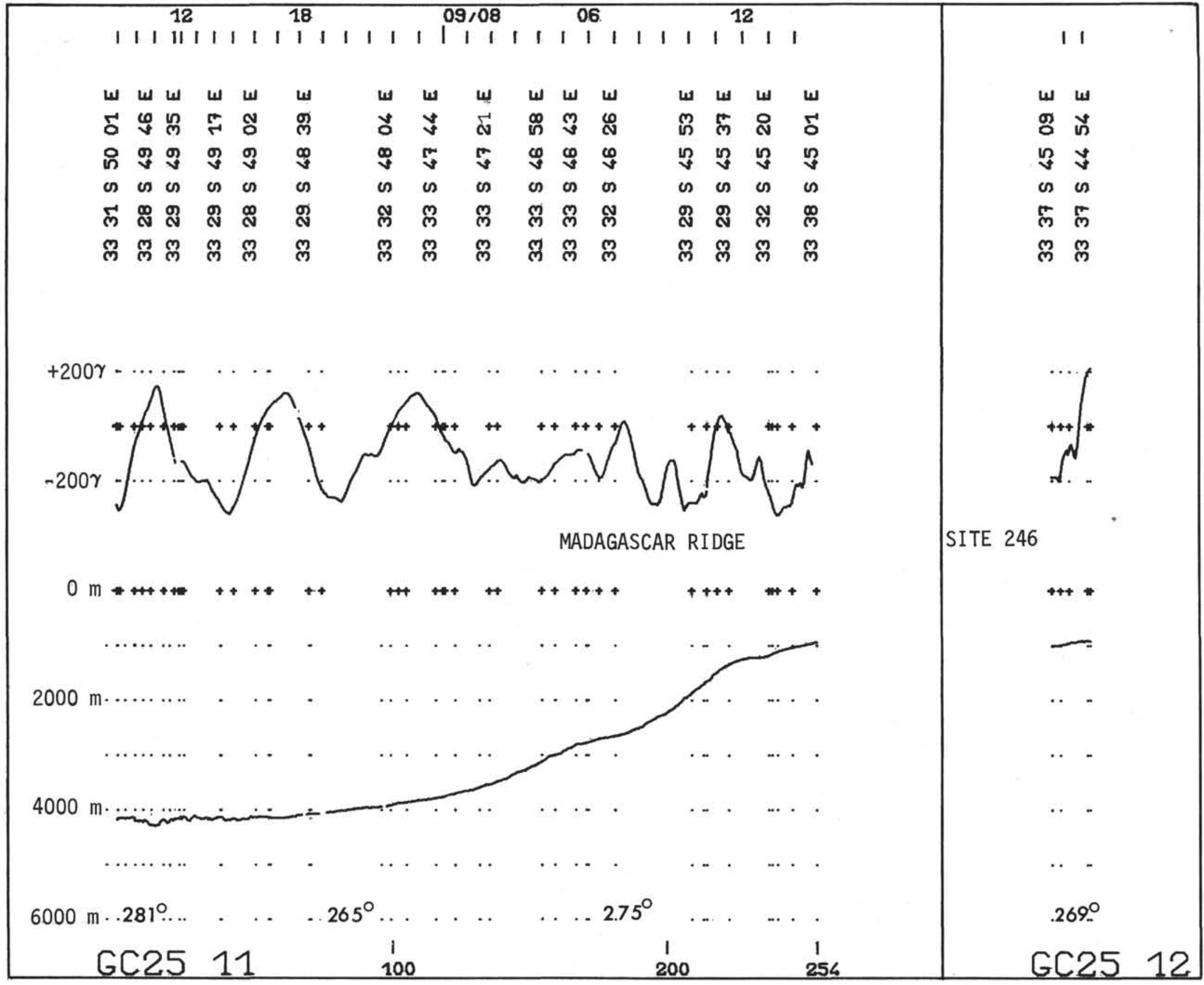

Figure 2. (Continued) 


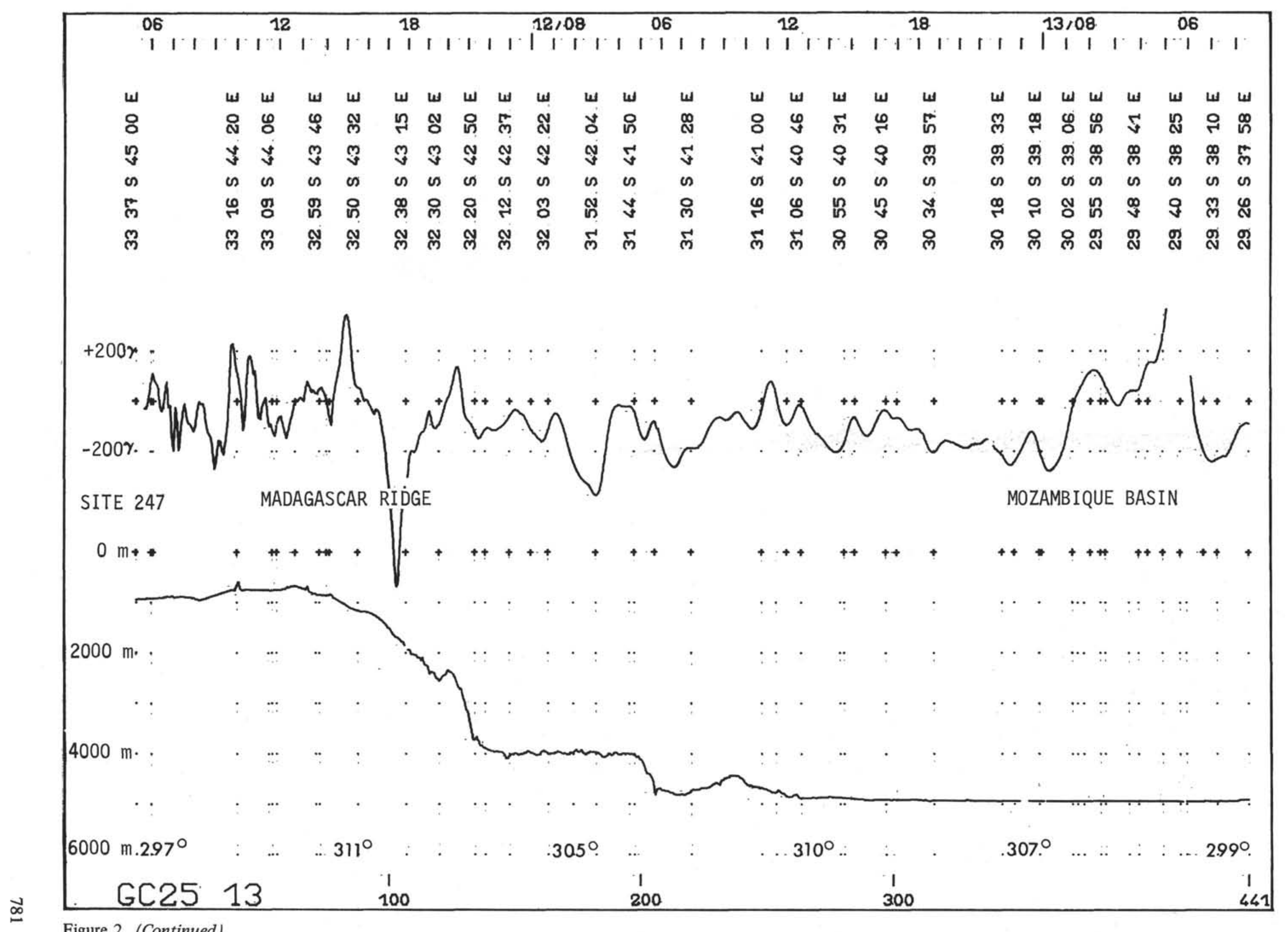




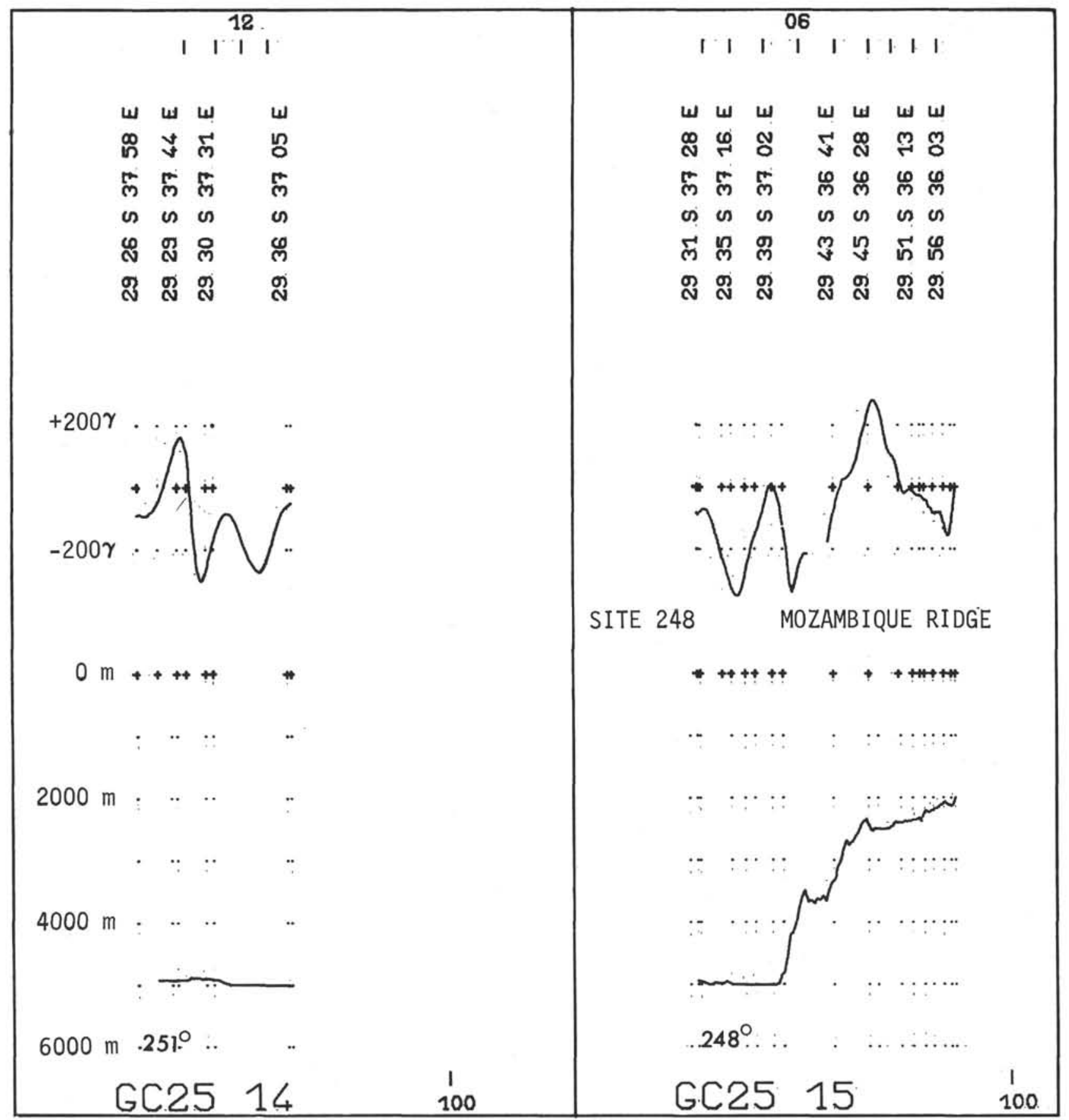




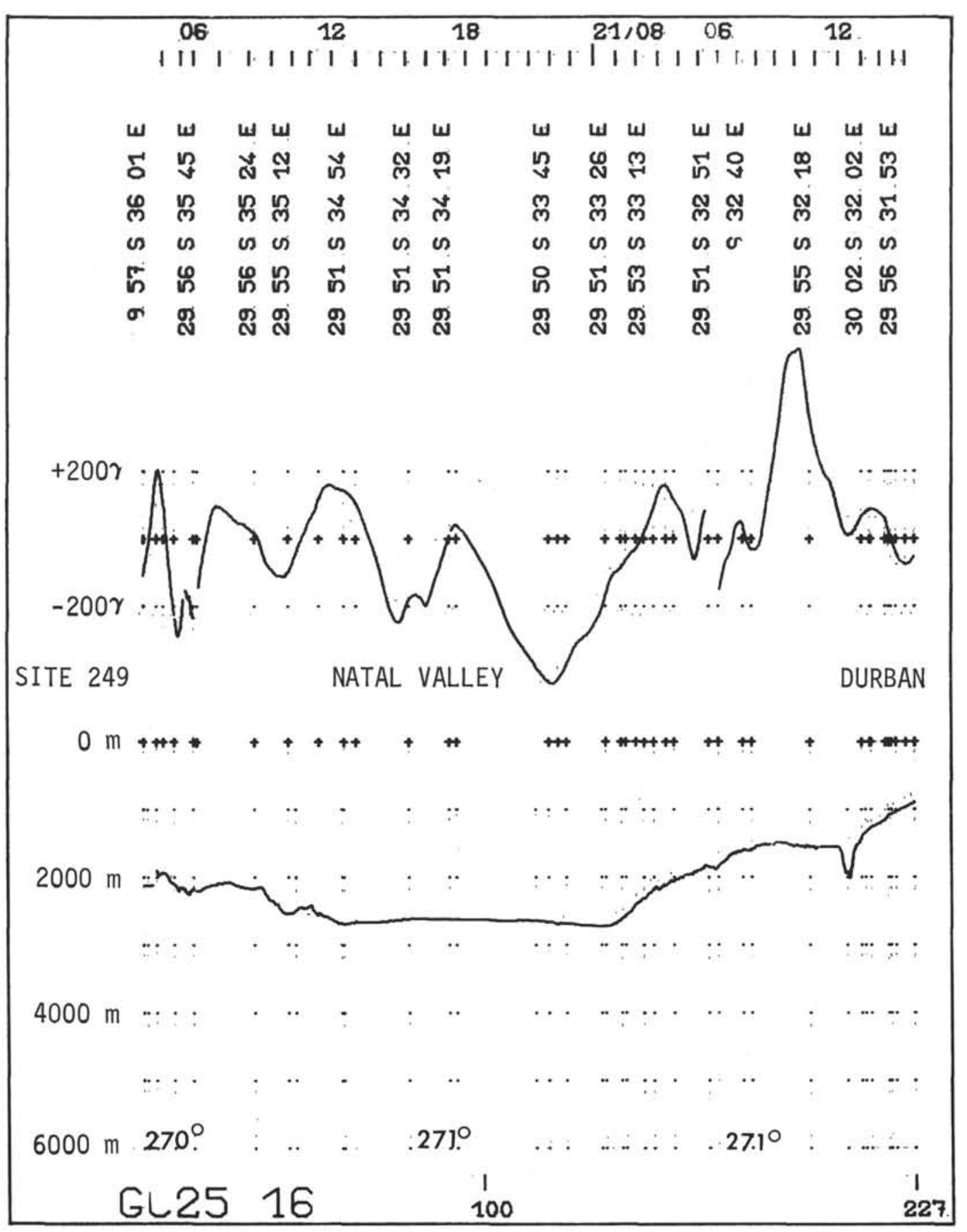

Figure 2. (Continued) 

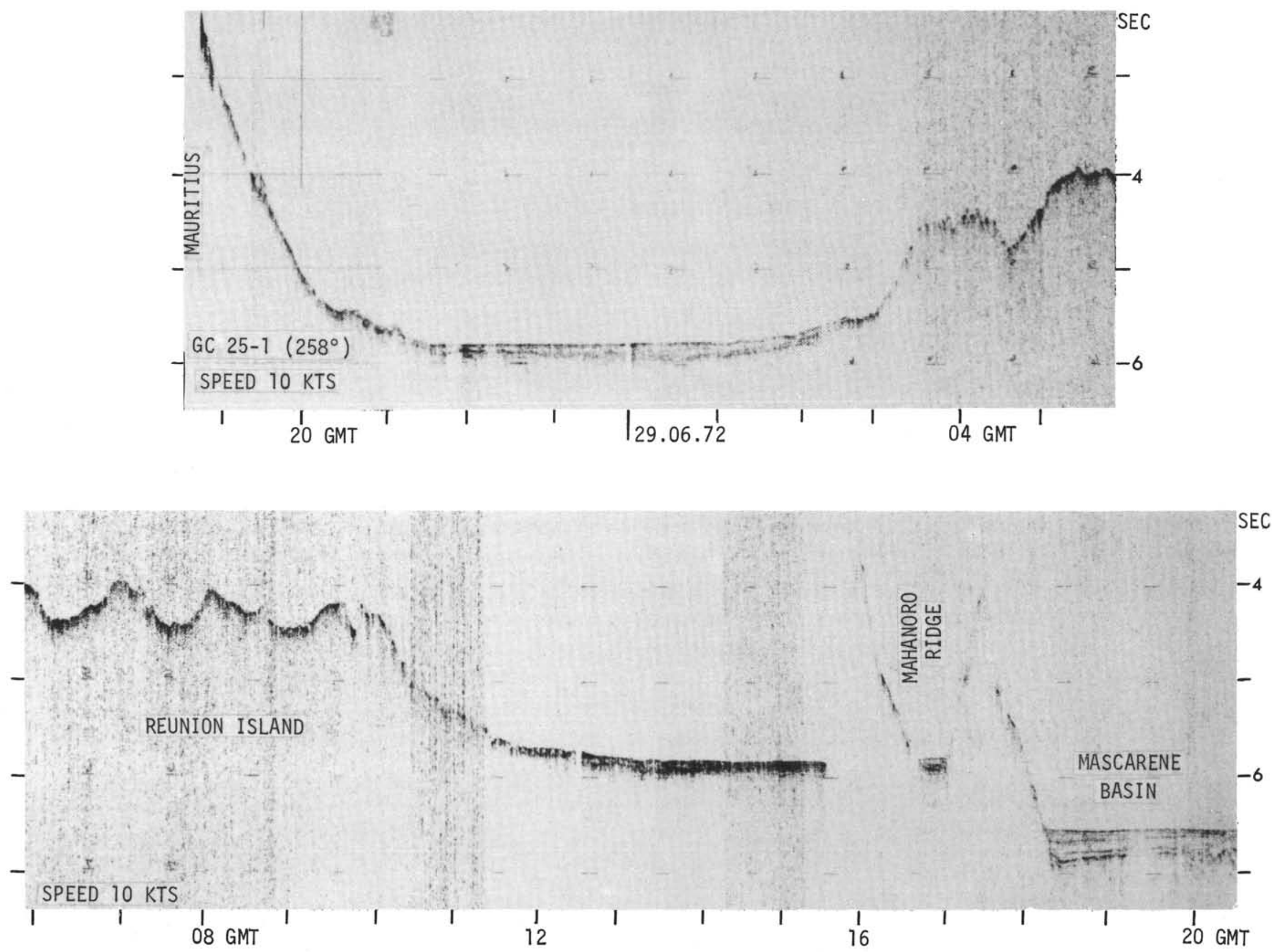

Figure 3. Glomar Challenger, DSDP Leg 25, 10-sec-sweep seismic reflection profiles displayed as a function of time. 

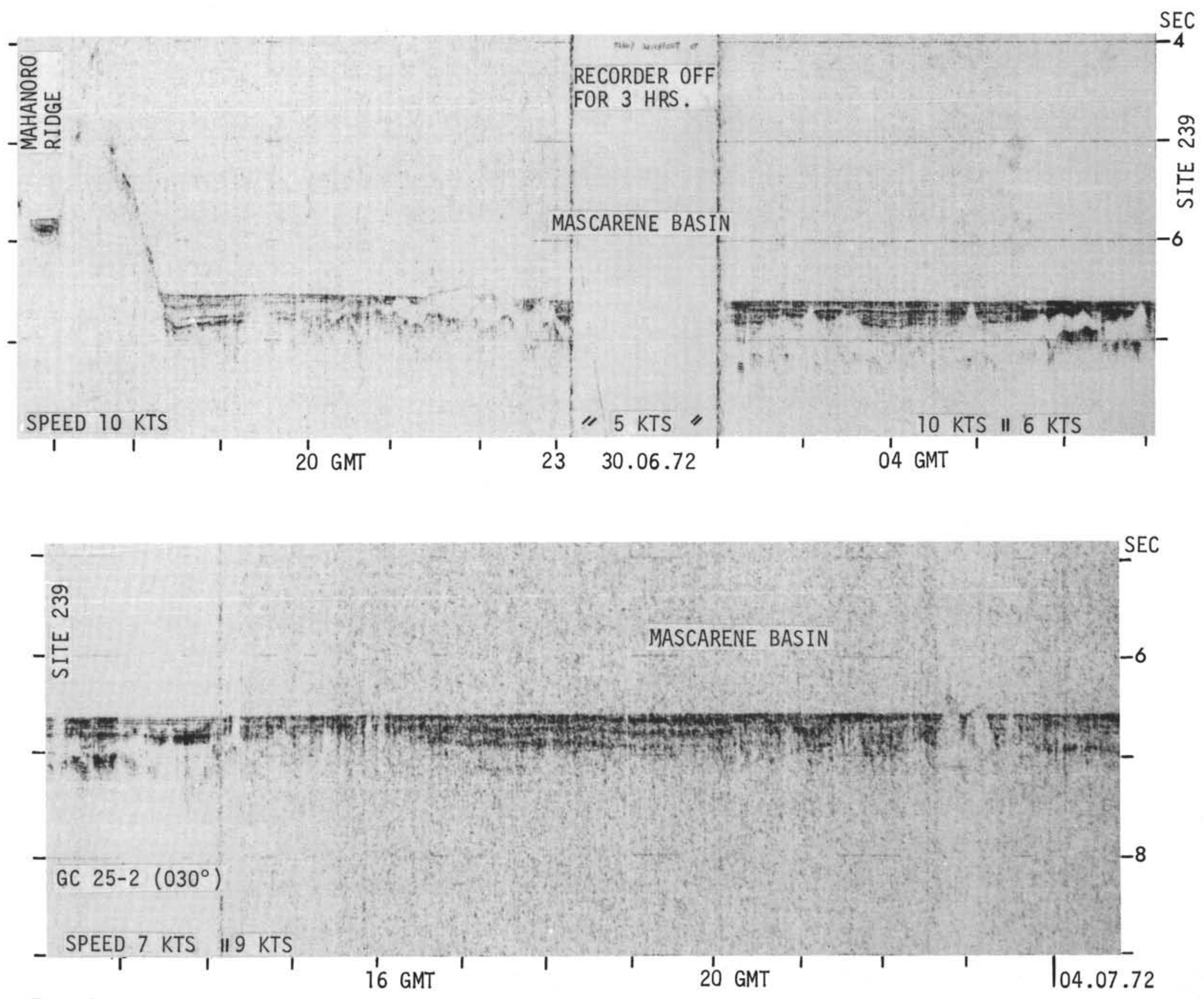

Figure 3. (Continued) 

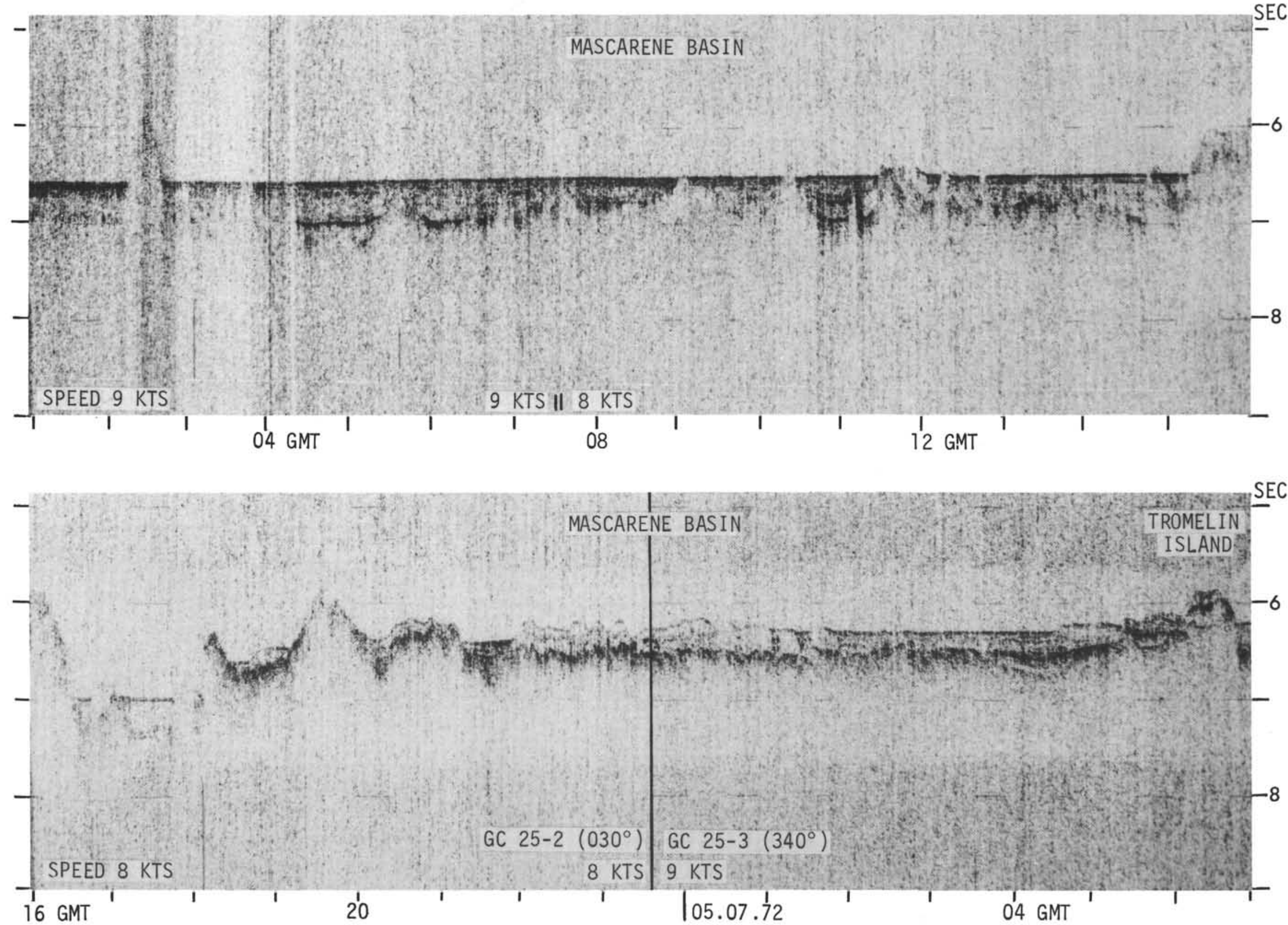

Figure 3. (Continued) 
SEC.

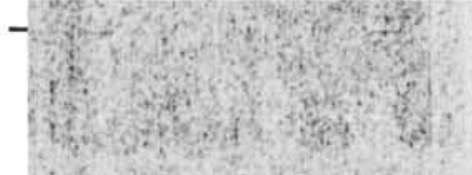

MASCARENE BASIN
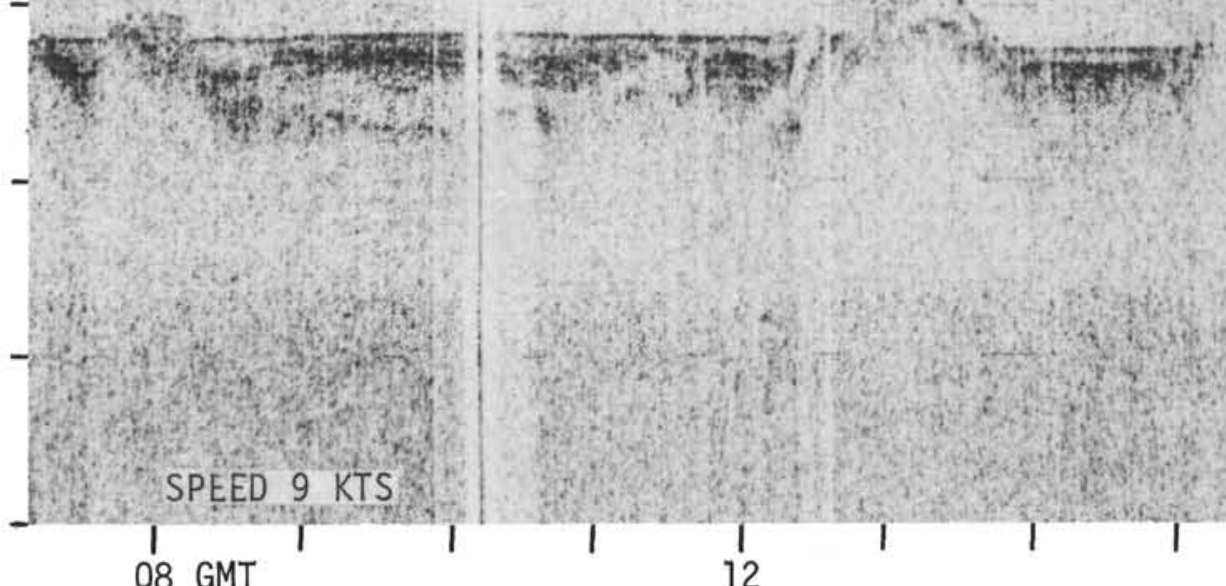

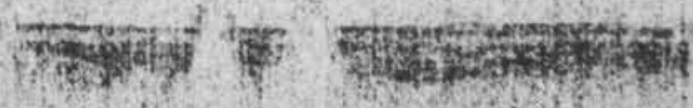

\section{is}
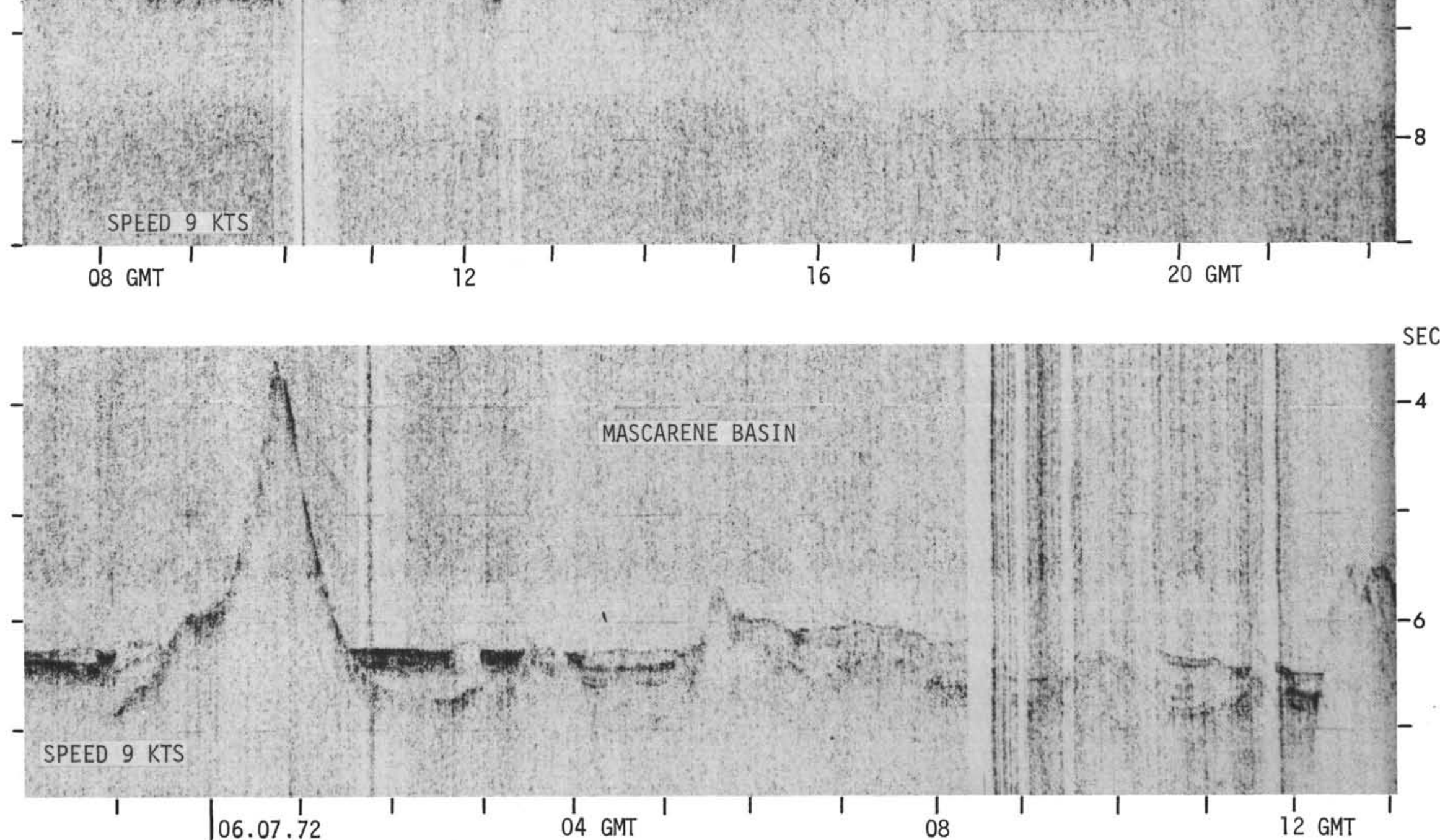

SEC. 


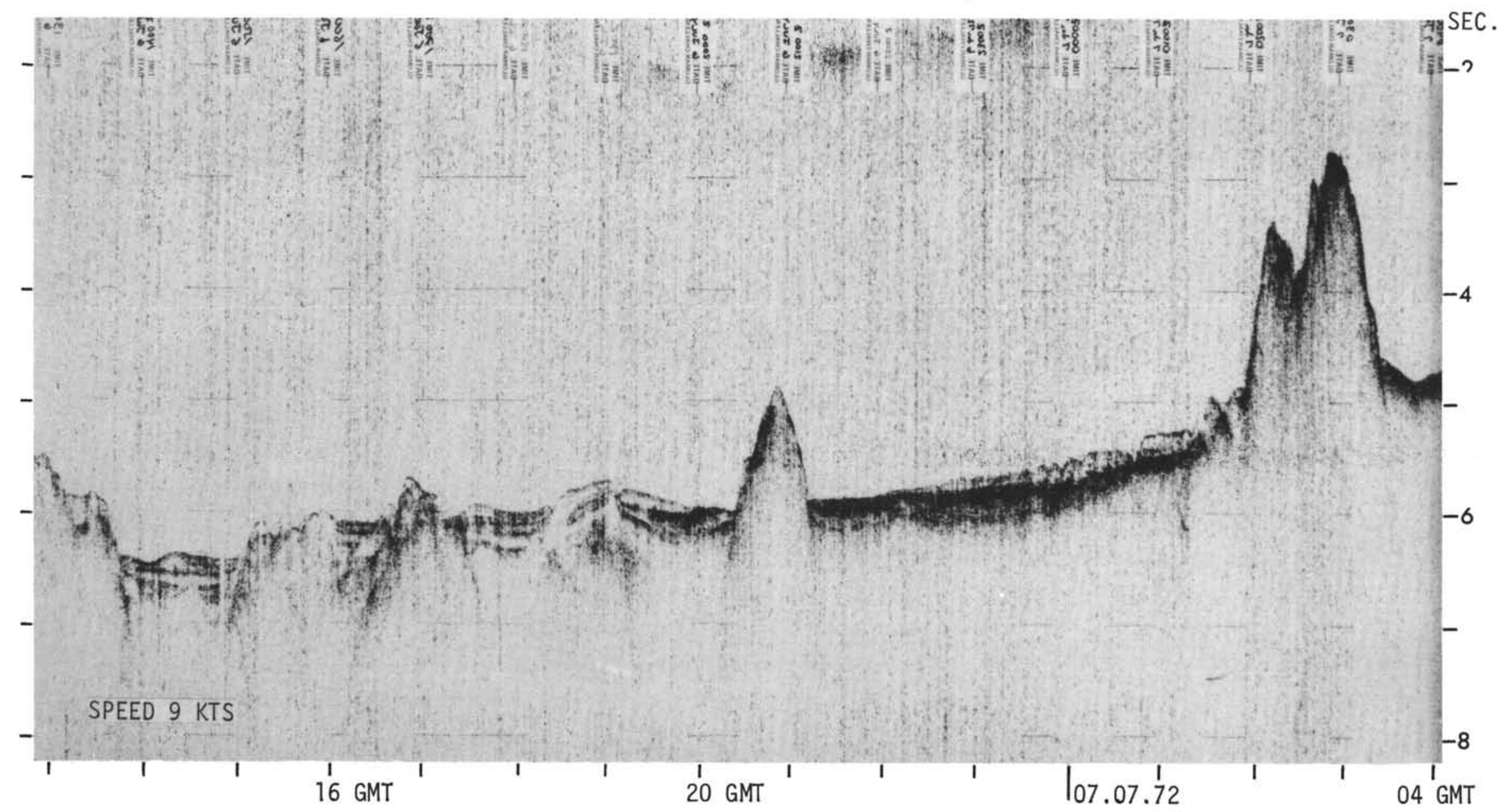

Figure 3. (Continued) 


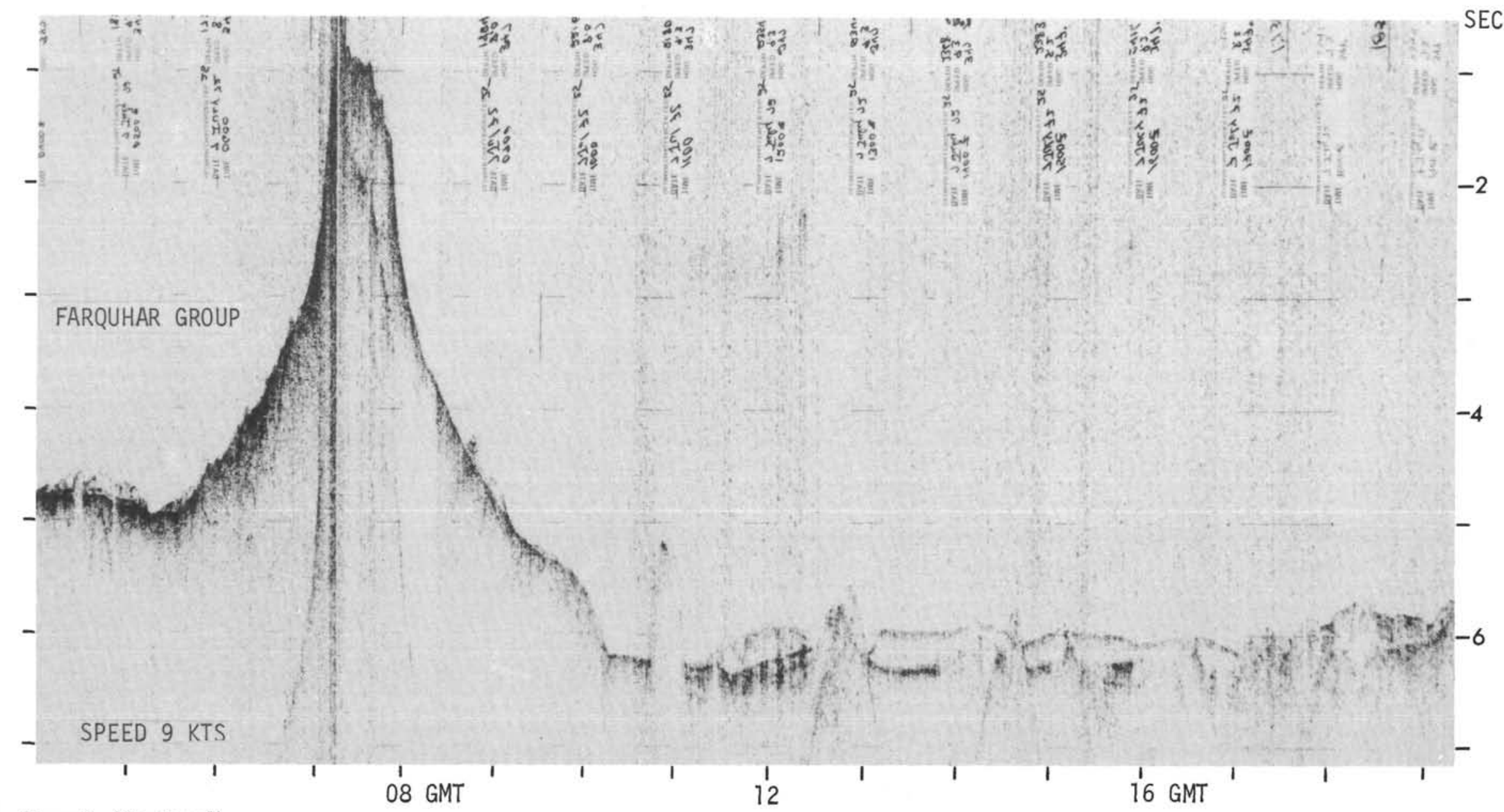

Figure 3. (Continued) 

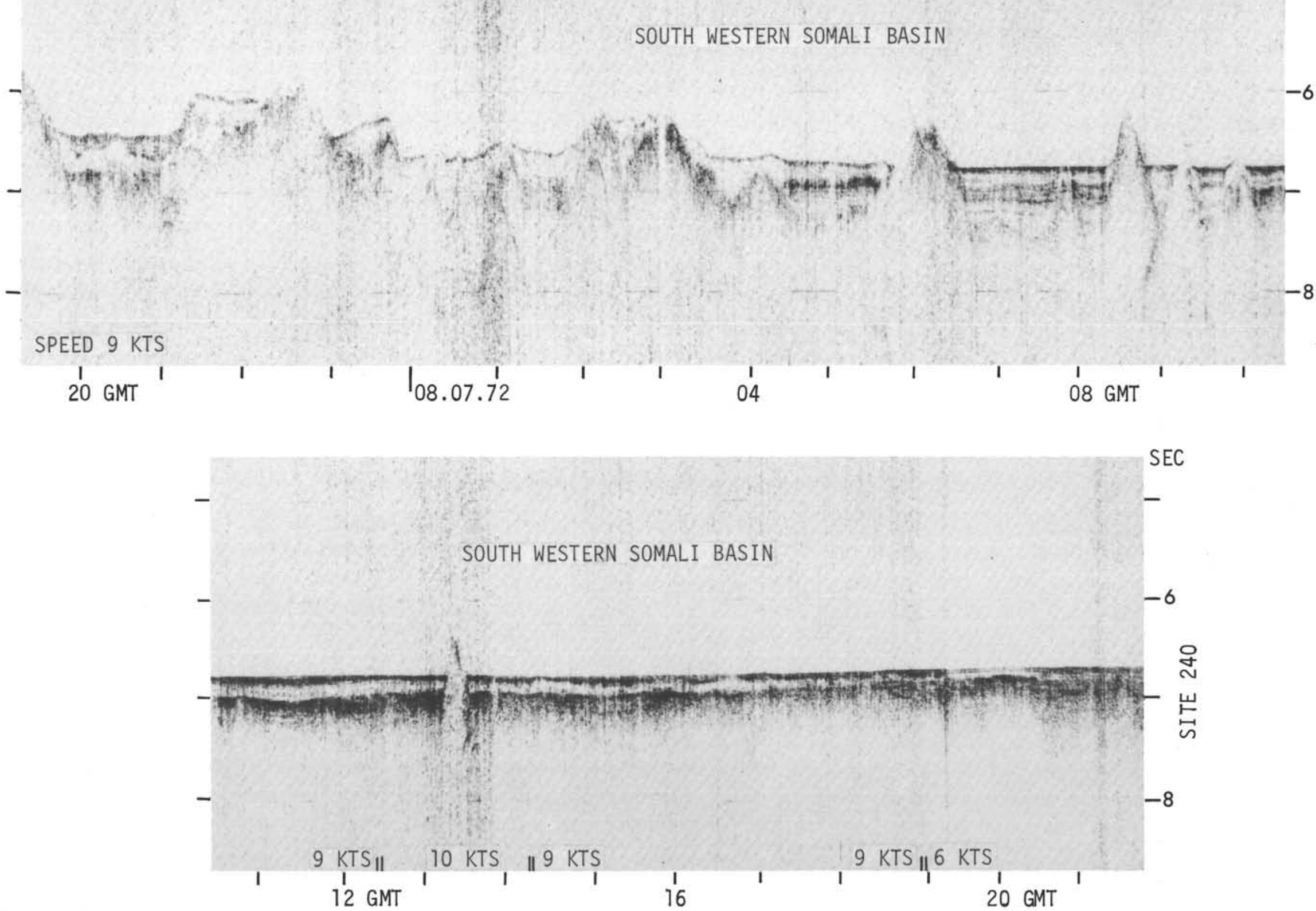

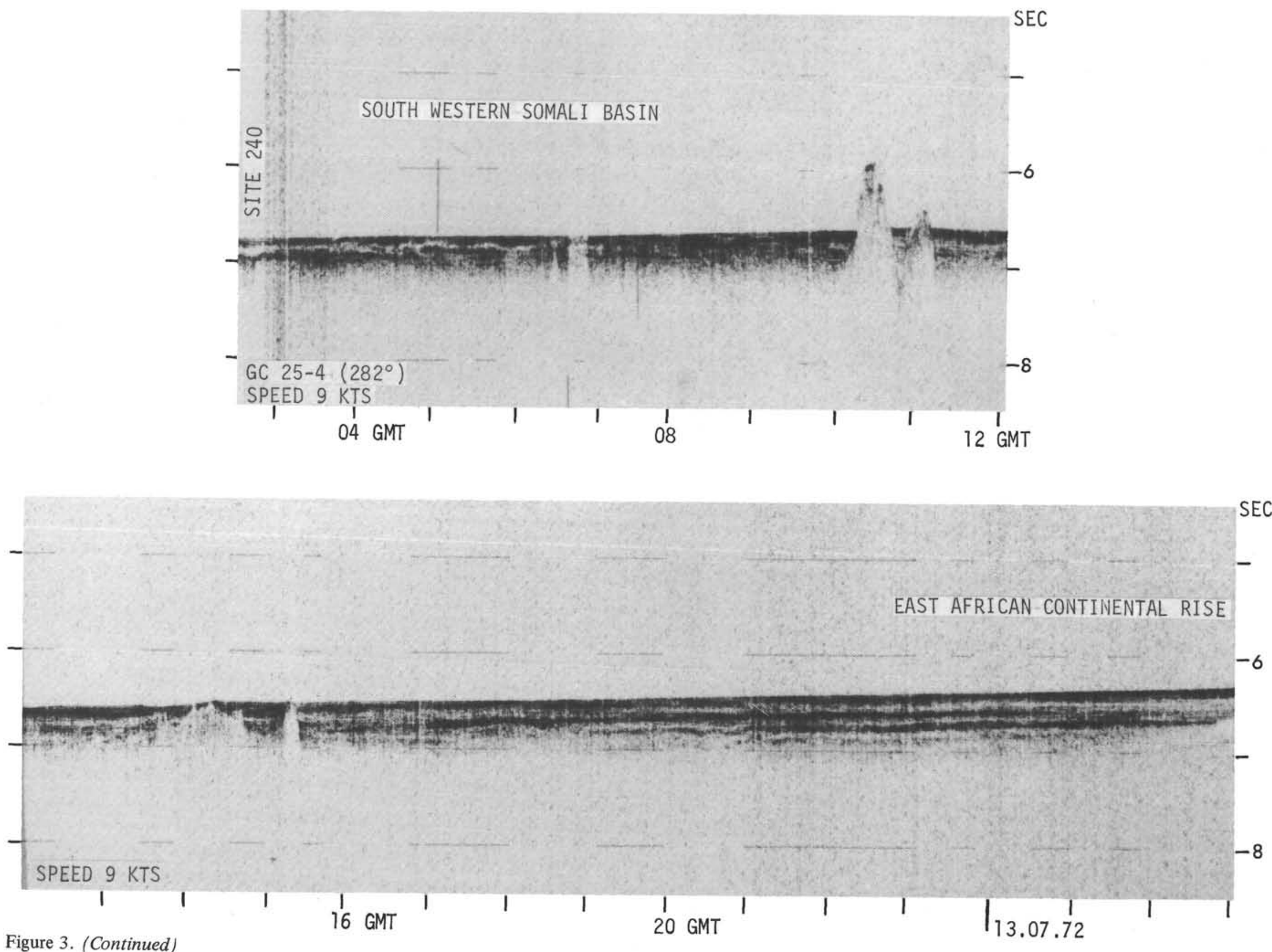

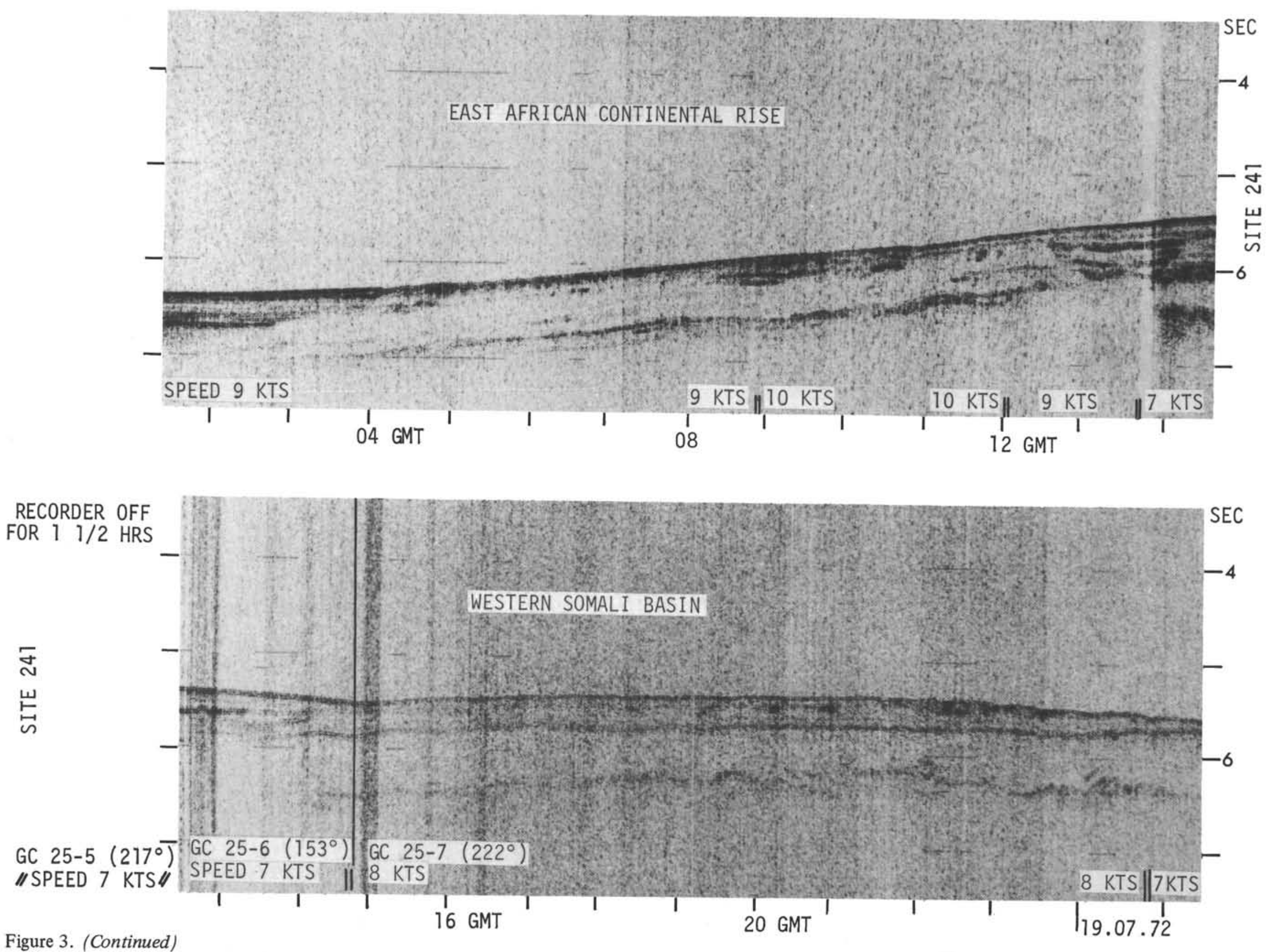

Figure 3. (Continued) 

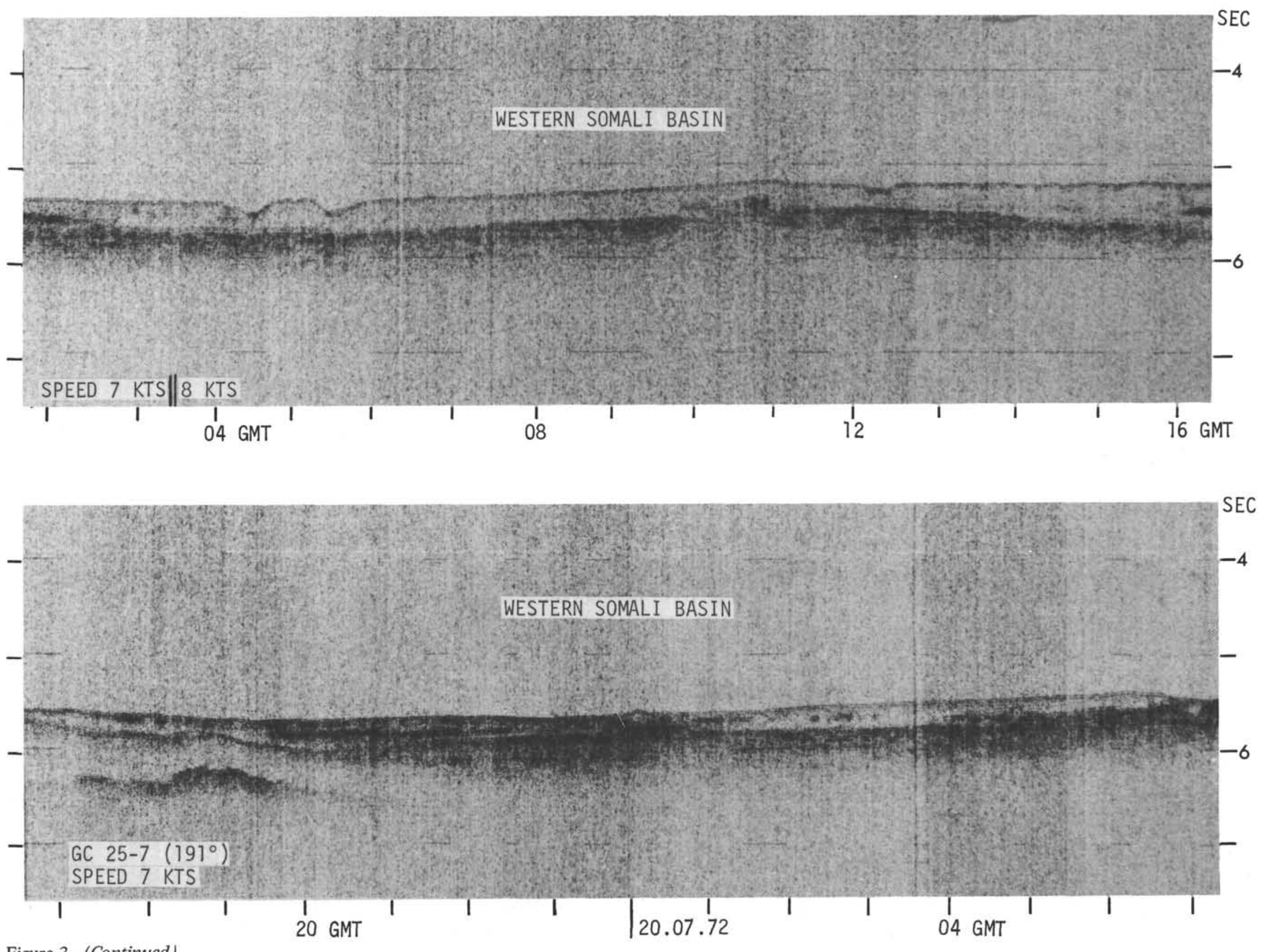

Figure 3. (Continued) 

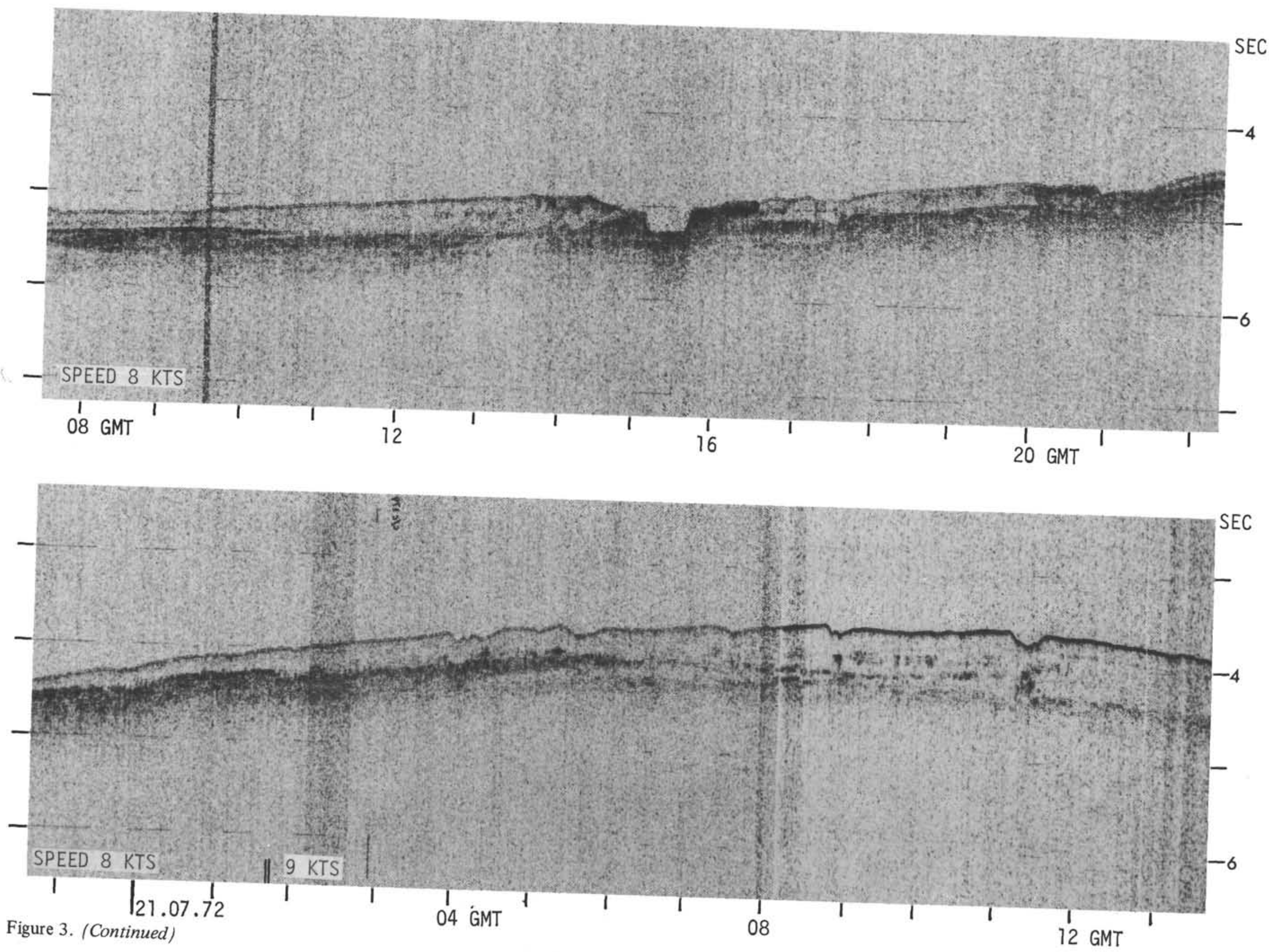

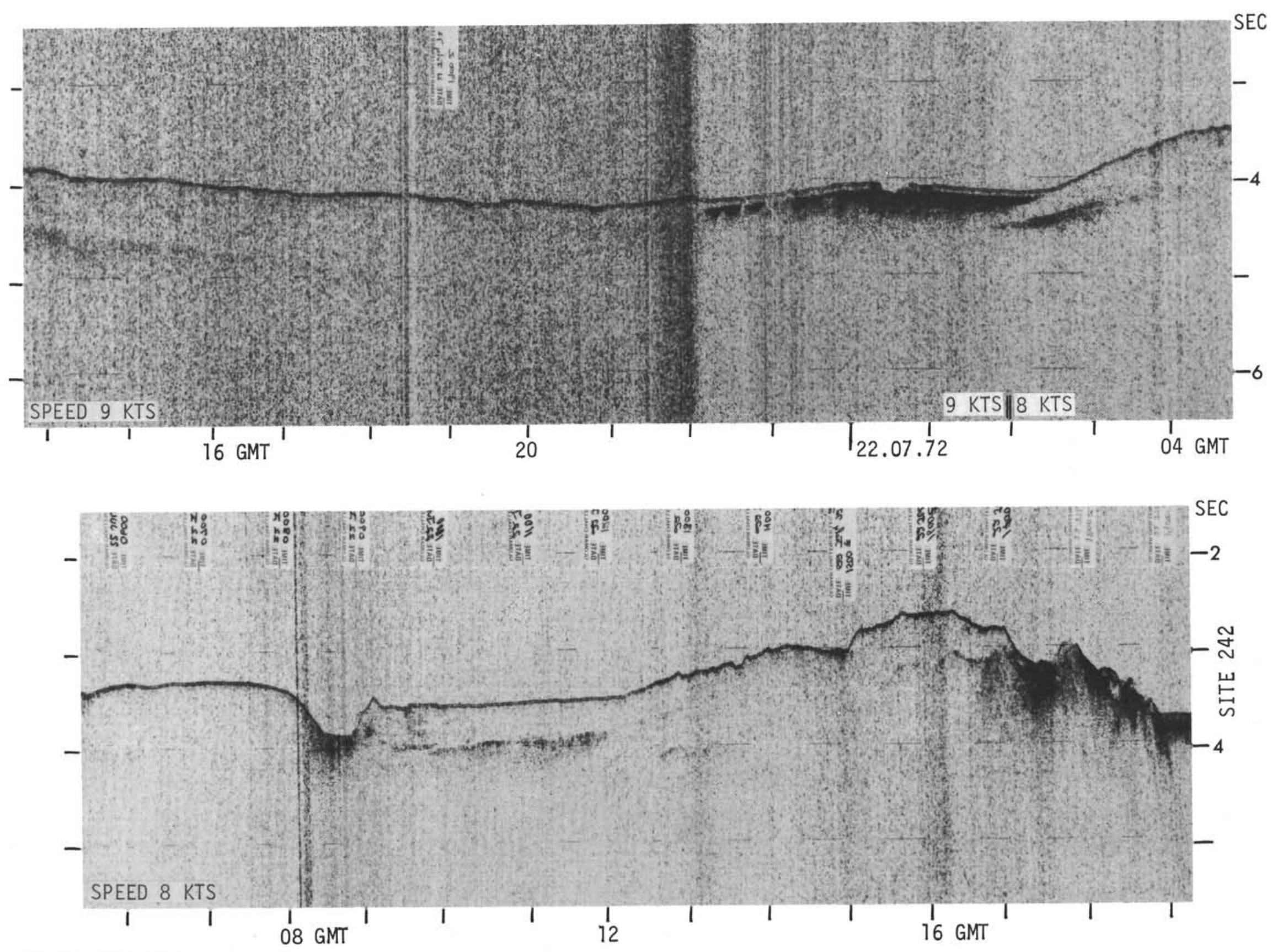

Figure 3. (Continued) 

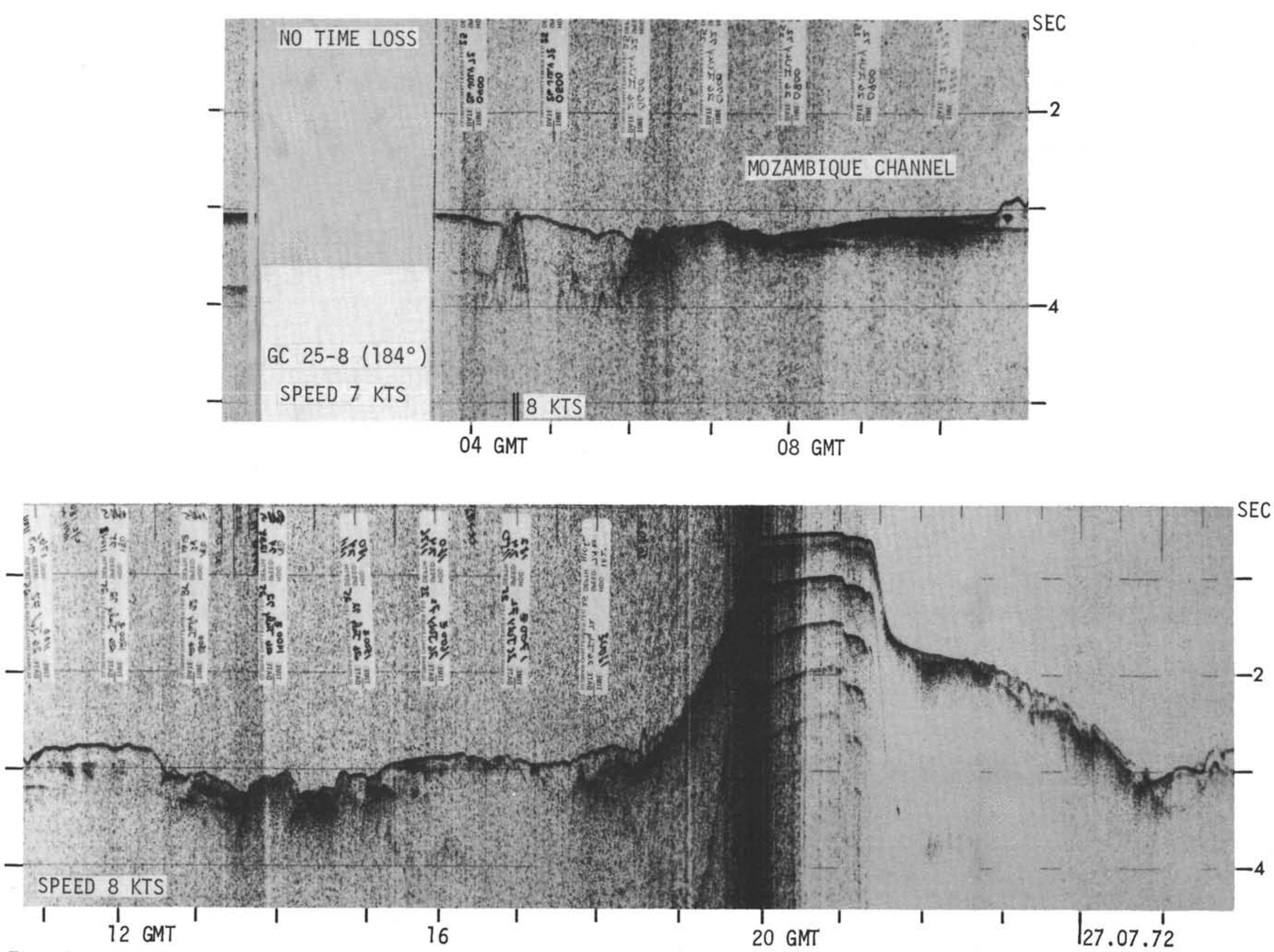

Figure 3. (Continued) 

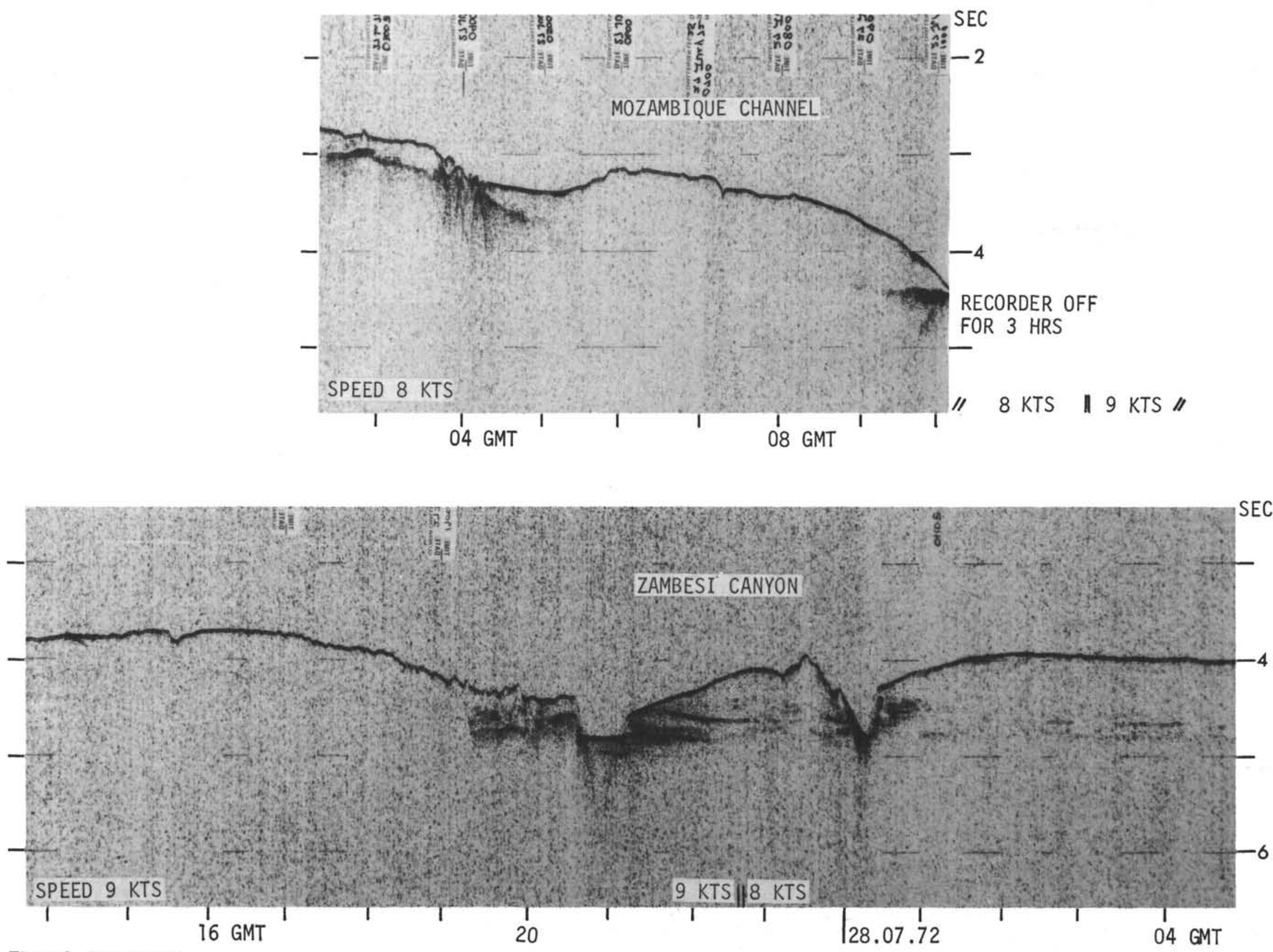

Figure 3. (Continued) 

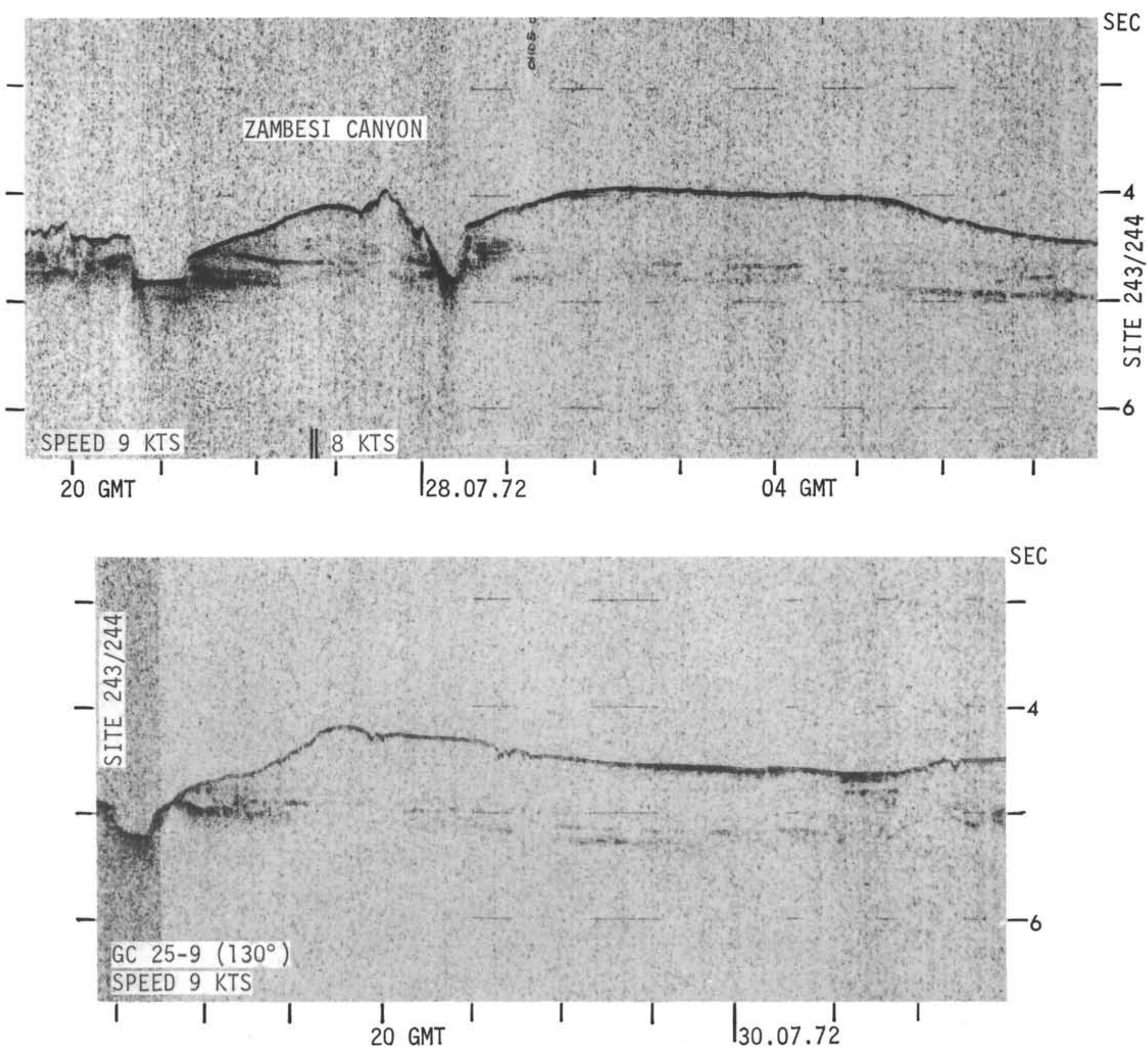

Figure 3. (Continued) 

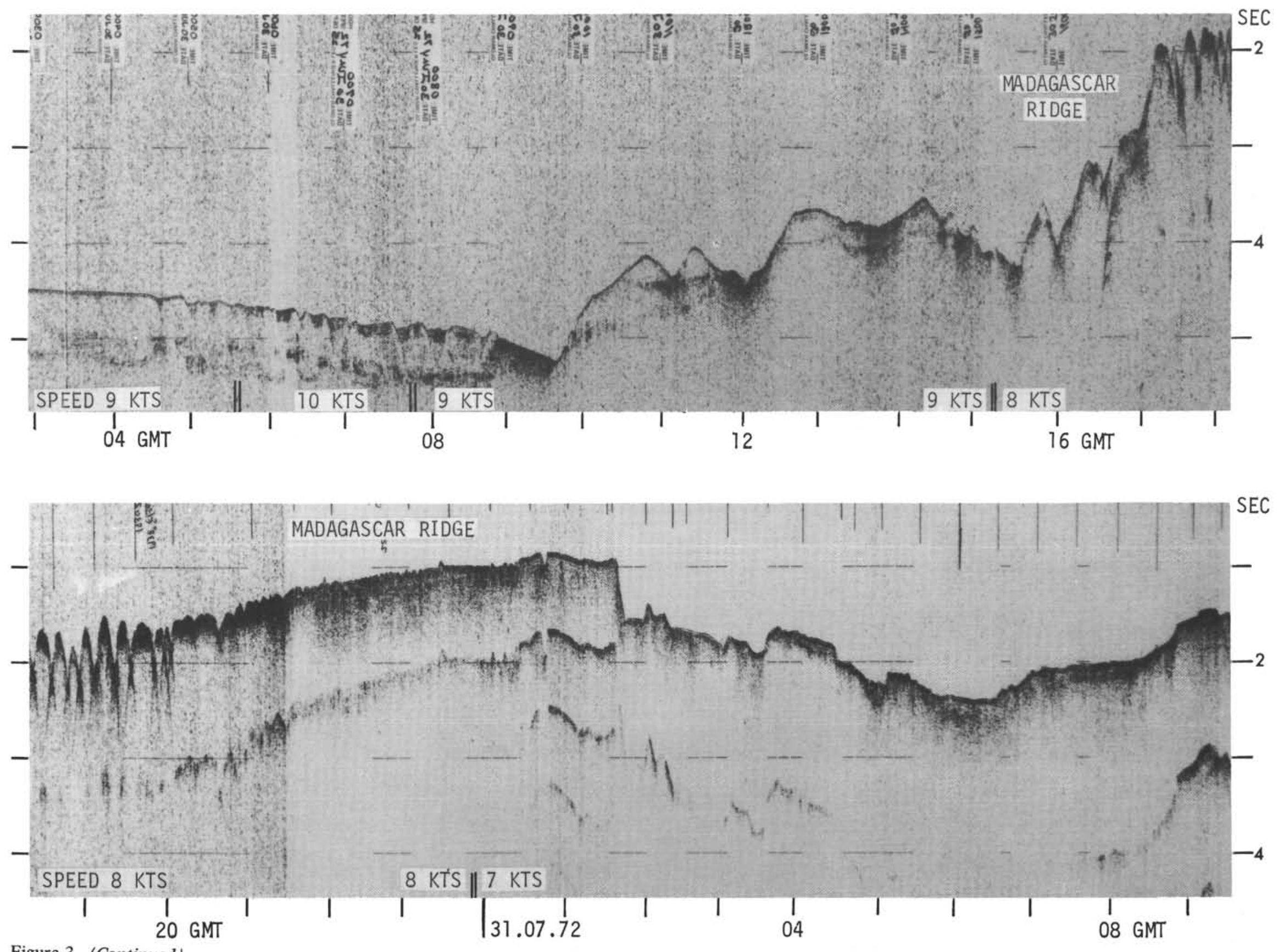

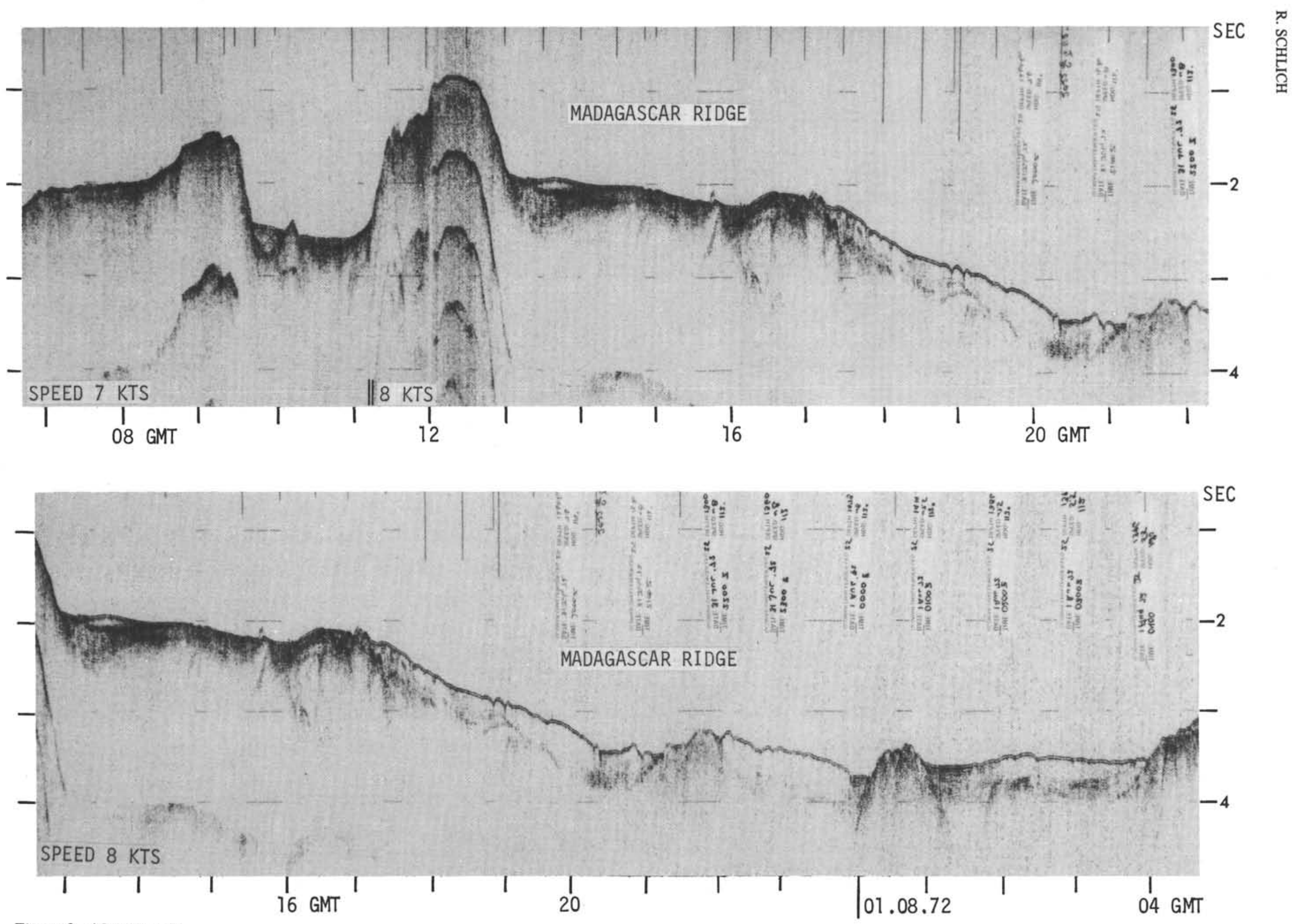

Figure 3. (Continued) 

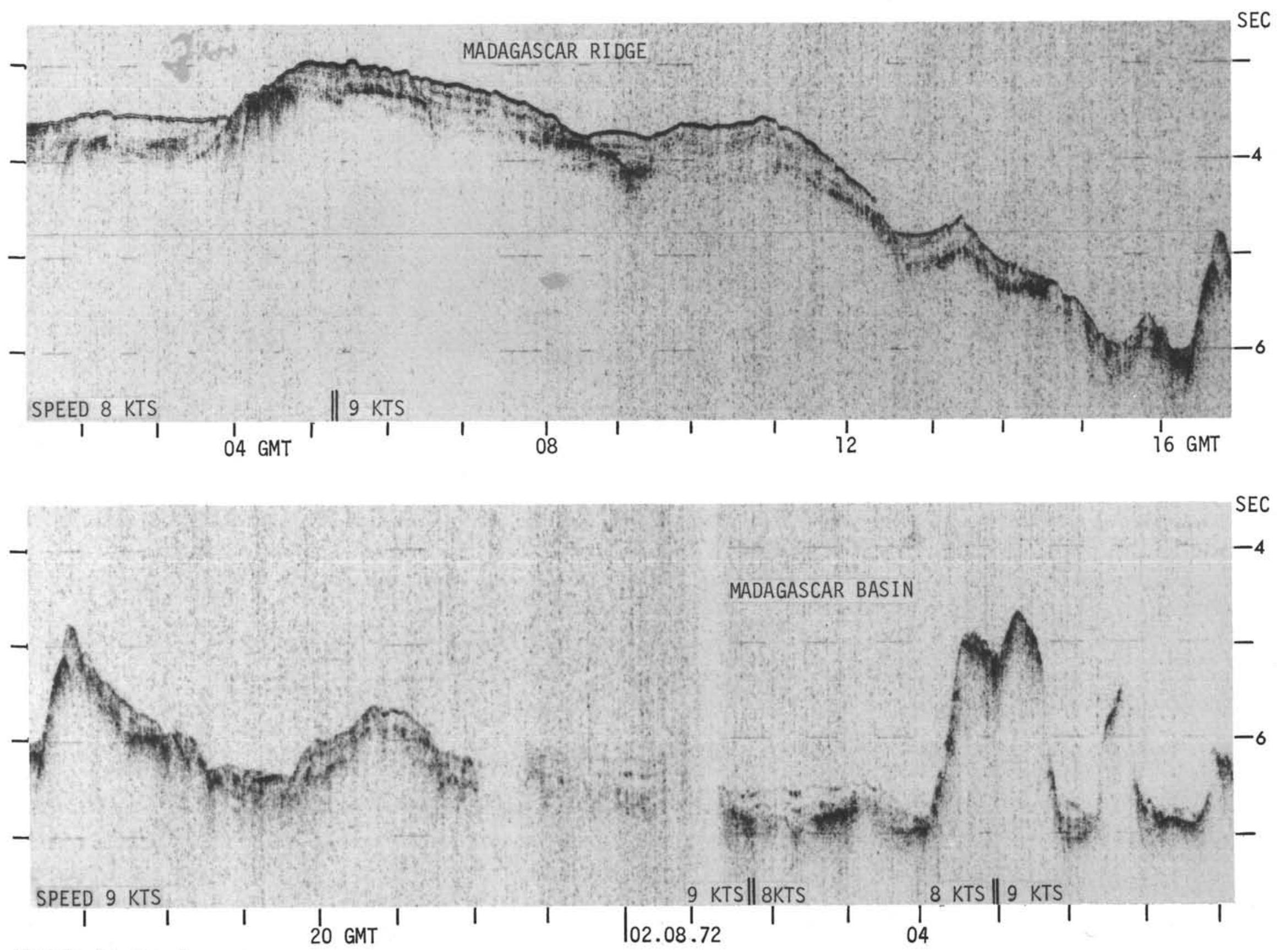

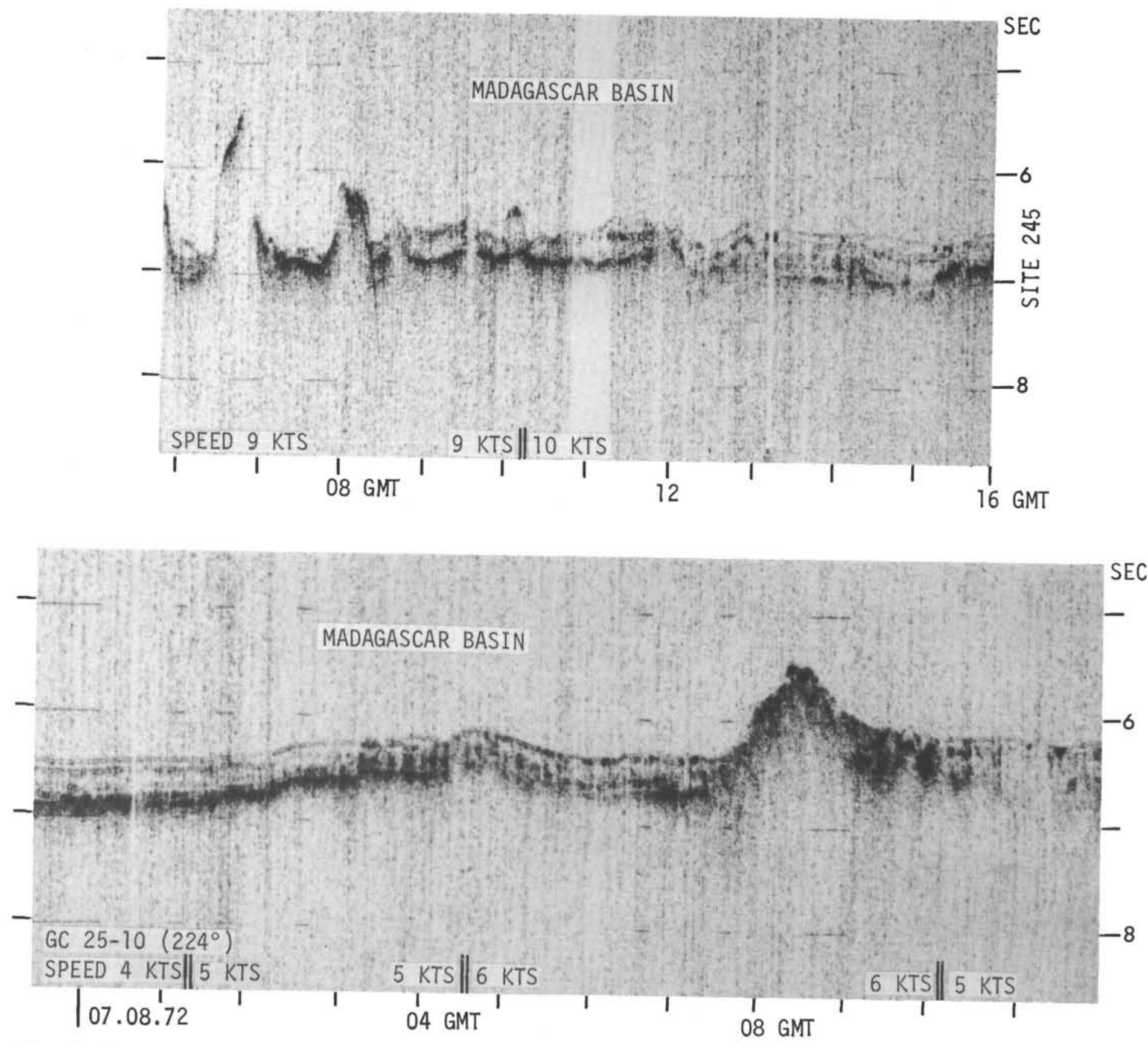

Figure 3. (Continued) 

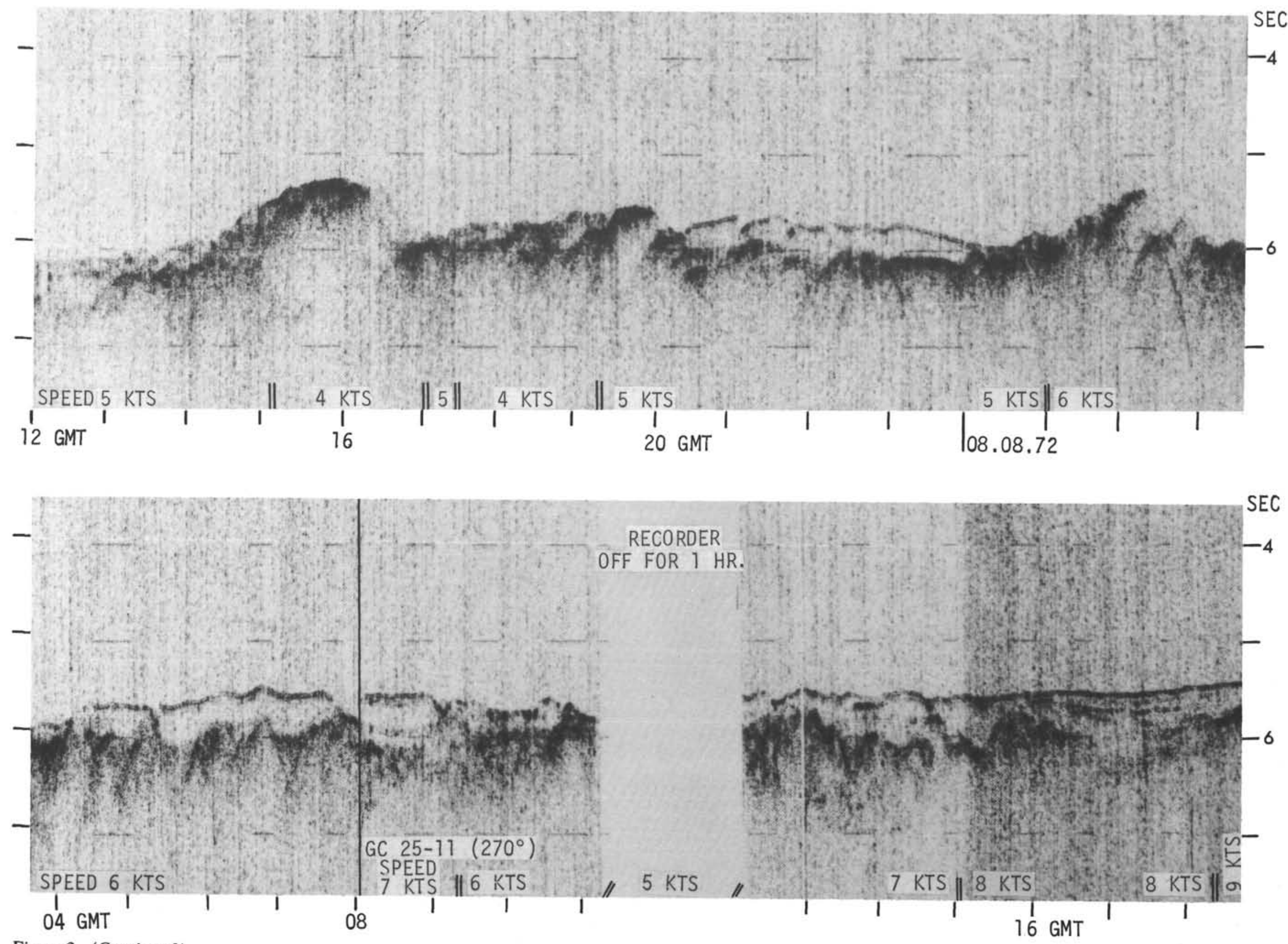

Figure 3. (Continued) 

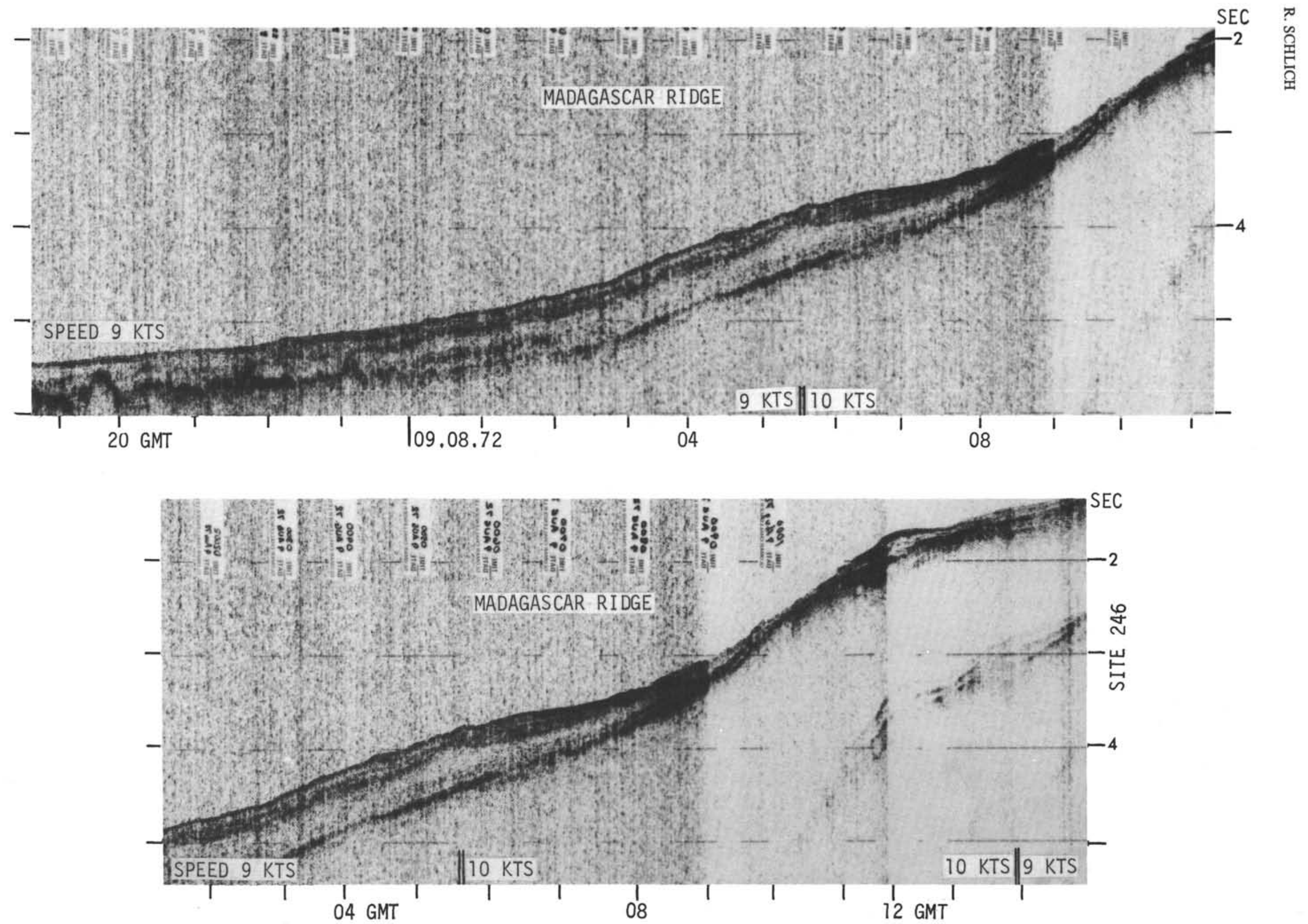

Figure 3. (Continued) 

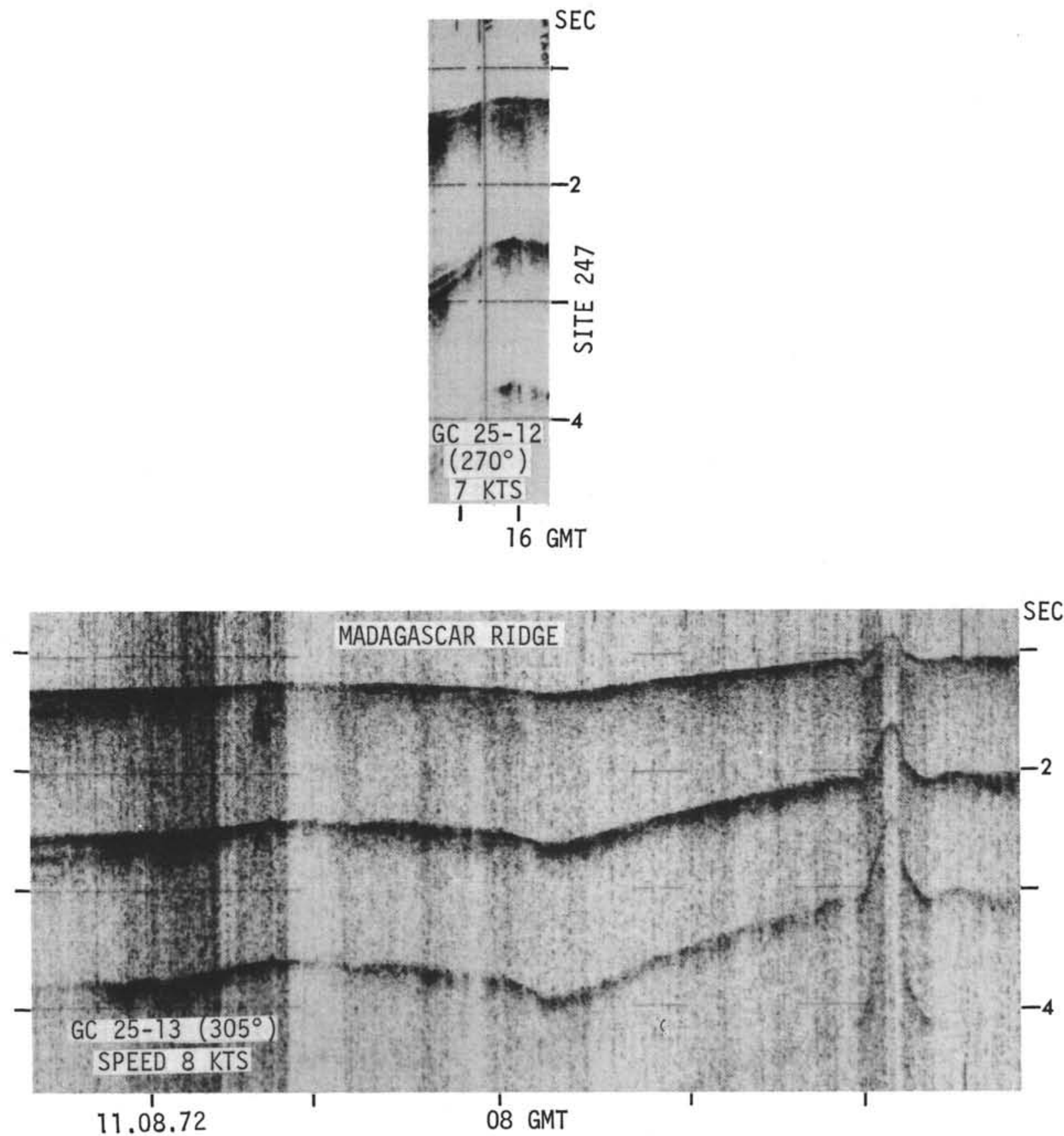


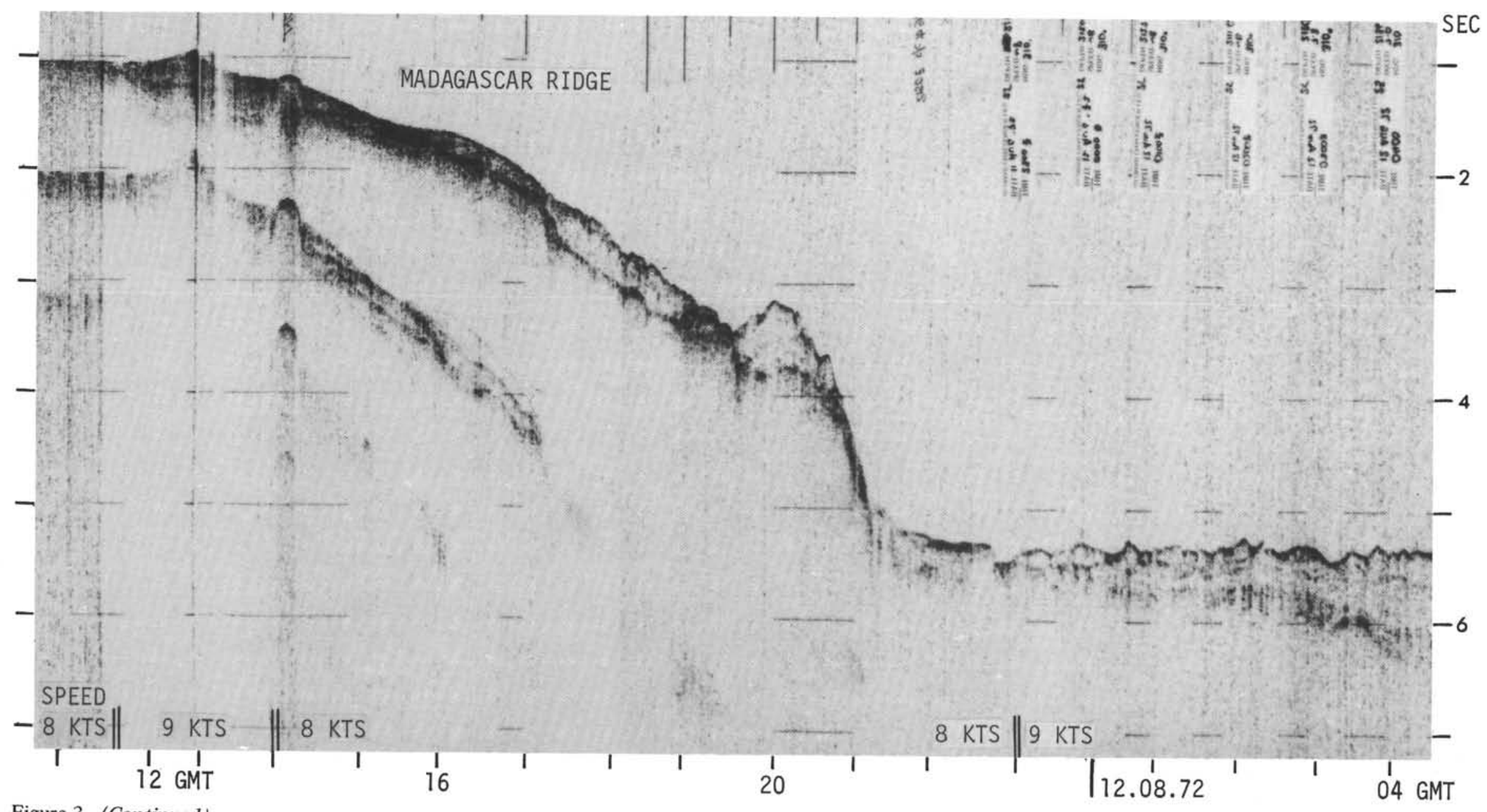

Figure 3. (Continued) 

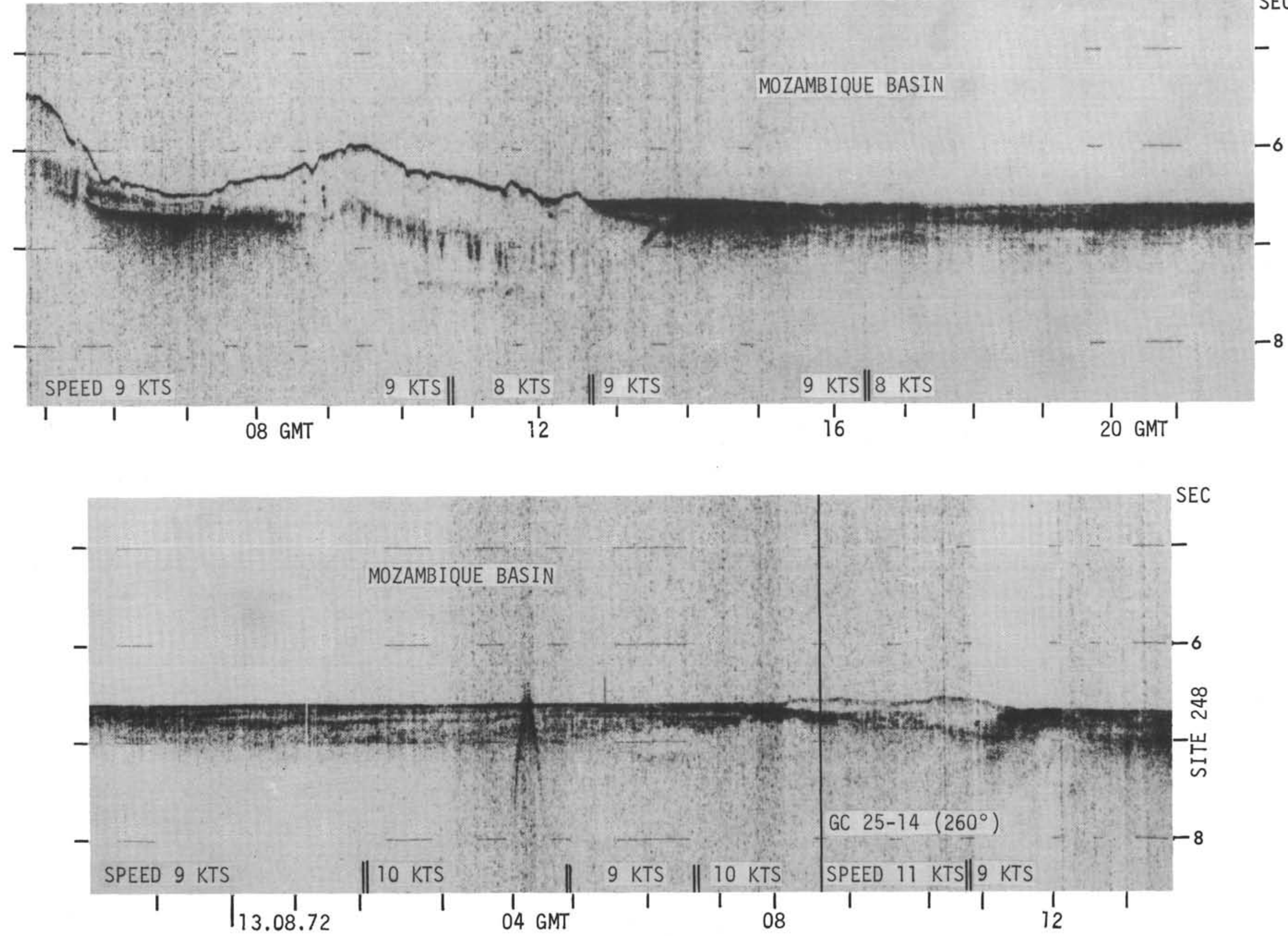


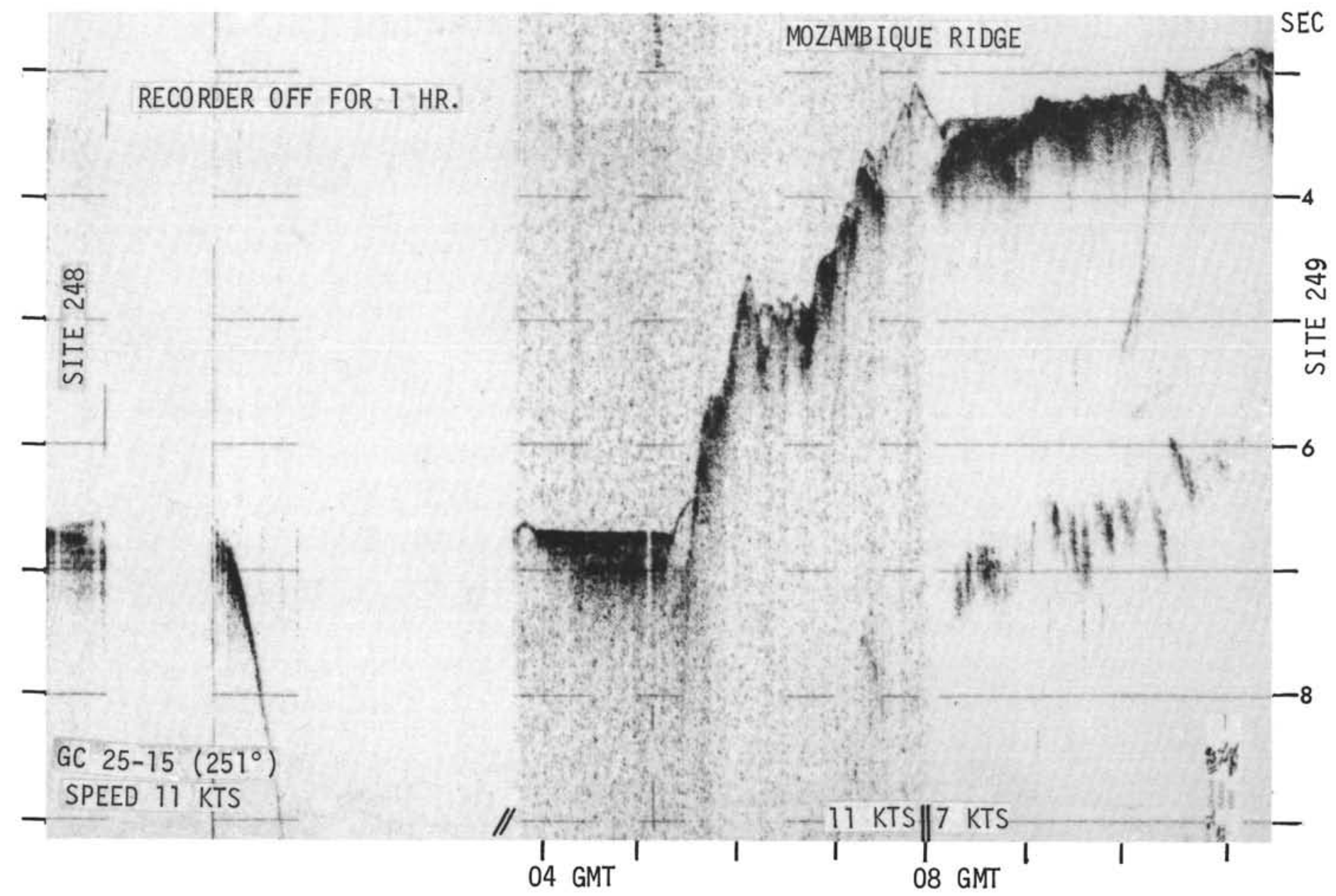

Figure 3. (Continued) 

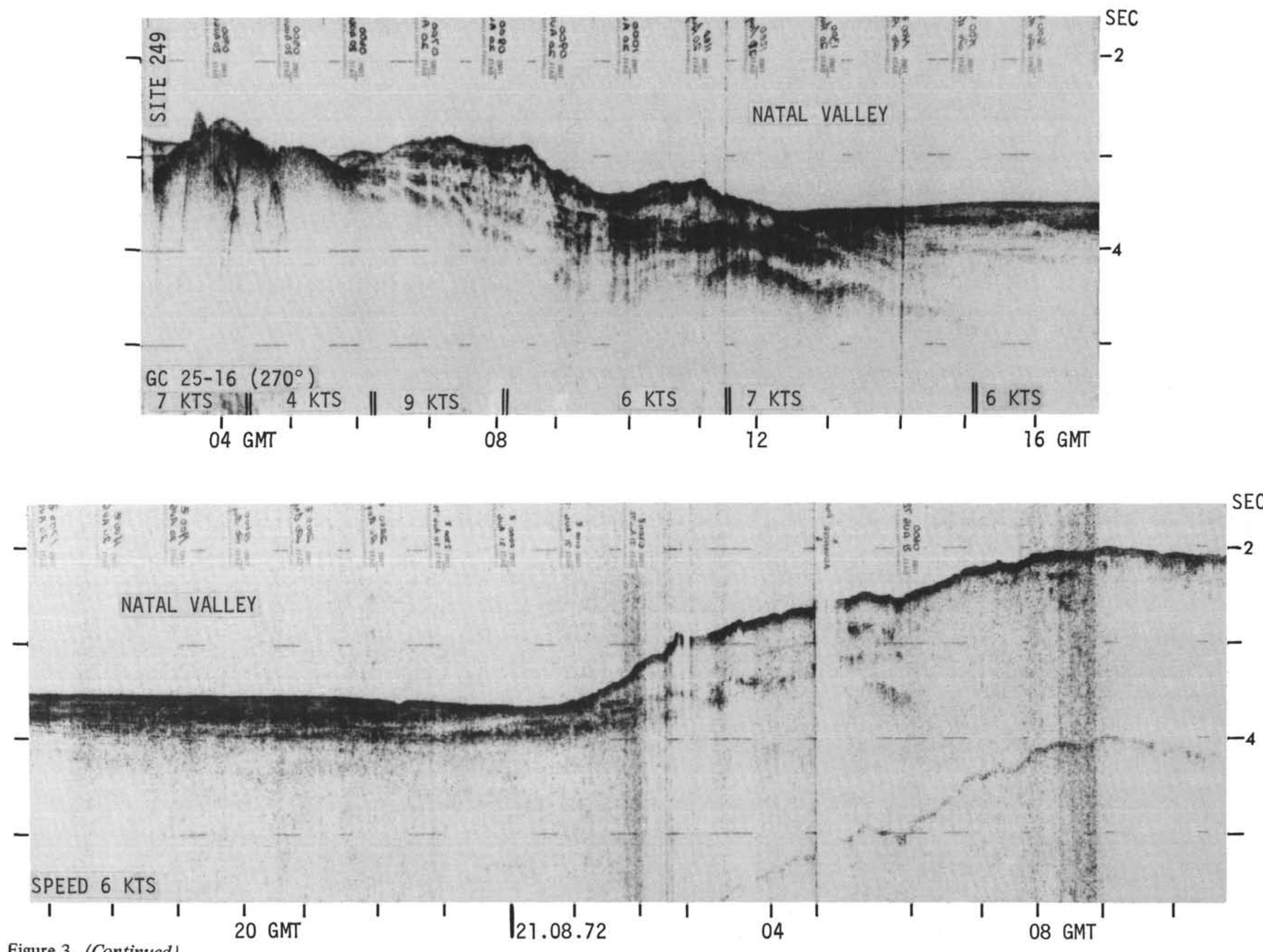


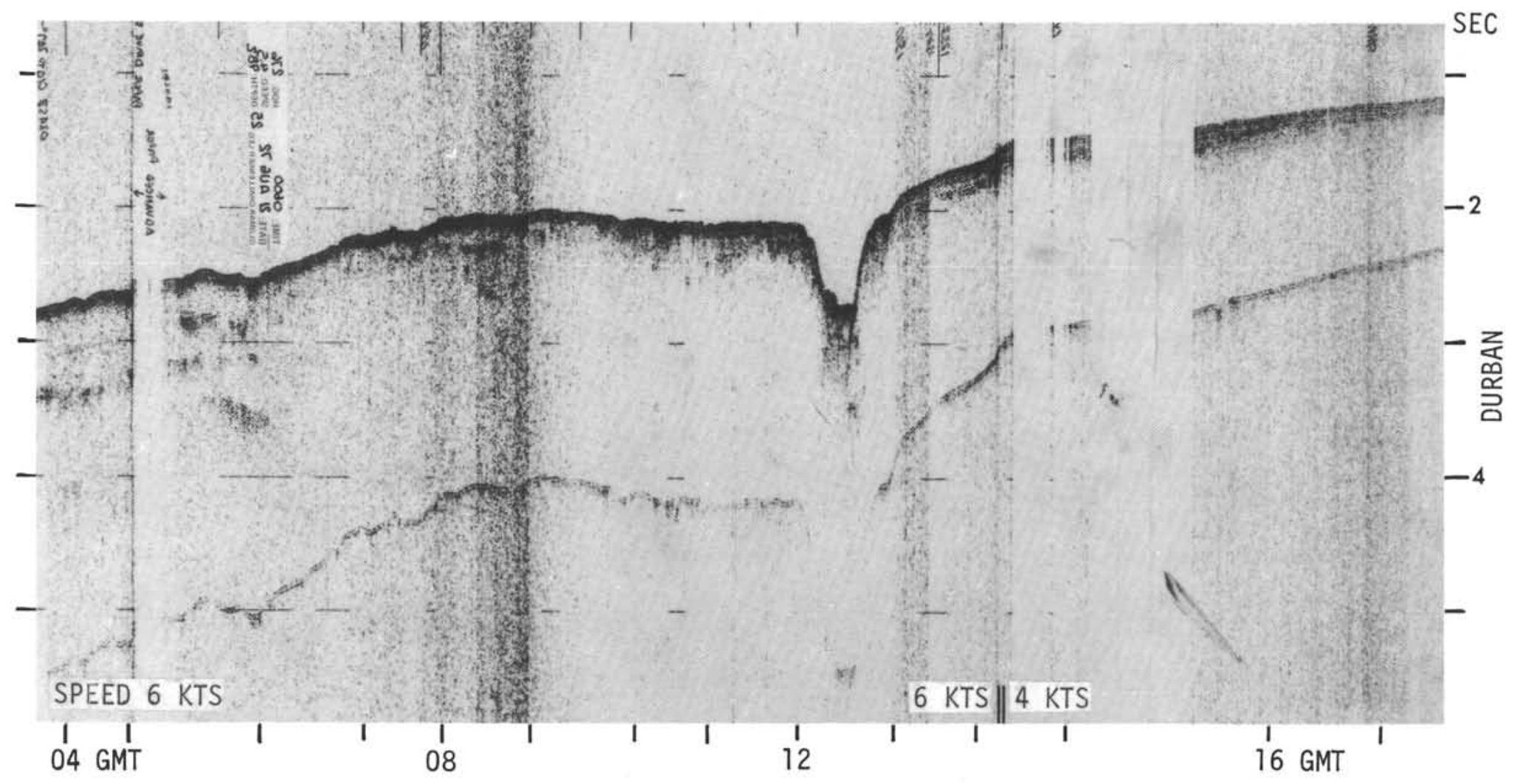

Figure 3. (Continued) 
TABLE

Glomar Challenger, DSDP Leg 25, Navigation Data

\begin{tabular}{|c|c|c|c|c|c|c|c|c|c|c|c|}
\hline $\begin{array}{c}\text { DATE } \\
\text { (day/mo) }\end{array}$ & $\begin{array}{c}\text { GMT } \\
(\mathrm{hr} / \mathrm{min})\end{array}$ & $\begin{array}{l}\text { LONGITUDE } \\
\text { (deg/min) }\end{array}$ & $\begin{array}{l}\text { LATITUDE } \\
\text { (deg/min) }\end{array}$ & $\begin{array}{c}\text { DATE } \\
\text { (day/mo) }\end{array}$ & $\begin{array}{c}\text { GMT } \\
(\mathrm{hr} / \mathrm{min})\end{array}$ & $\begin{array}{l}\text { LONGITUDE } \\
\text { (deg/min) }\end{array}$ & $\begin{array}{l}\text { LATITUDE } \\
(\mathrm{deg} / \mathrm{min})\end{array}$ & $\begin{array}{r}\text { DIST } \\
(\mathrm{nmi})\end{array}$ & $\begin{array}{l}\text { CSE } \\
\left({ }^{\circ}\right)\end{array}$ & $\begin{array}{l}\text { SPEED } \\
(k t)\end{array}$ & $\begin{array}{l}\text { DISTT } \\
(n m i)\end{array}$ \\
\hline 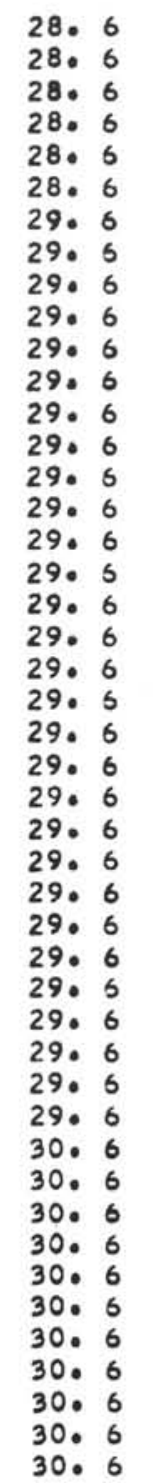 & 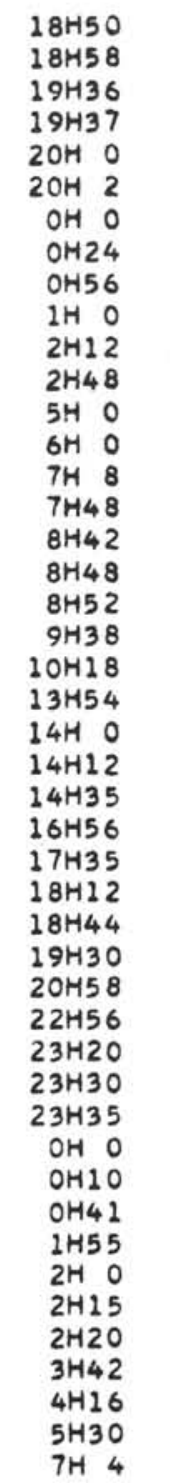 & $\begin{array}{l}57023 \cdot 4 \\
57022 \cdot 0 \\
57015 \cdot 7 \\
57015 \cdot 5 \\
57011 \cdot 6 \\
57011 \cdot 3 \\
56031 \cdot 2 \\
56027 \cdot 2 \\
56021 \cdot 5 \\
56020 \cdot 8 \\
5608 \cdot 3 \\
5602 \cdot 1 \\
55038 \cdot 5 \\
55027 \cdot 8 \\
55016 \cdot 0 \\
5509 \cdot 3 \\
5500 \cdot 2 \\
54059 \cdot 1 \\
54058 \cdot 5 \\
54050 \cdot 8 \\
54044 \cdot 4 \\
5409 \cdot 2 \\
5408 \cdot 2 \\
5406 \cdot 2 \\
5402 \cdot 5 \\
53039 \cdot 1 \\
53032 \cdot 7 \\
53026 \cdot 6 \\
53021 \cdot 1 \\
53013 \cdot 5 \\
52059 \cdot 3 \\
52038 \cdot 4 \\
52036 \cdot 6 \\
52036 \cdot 6 \\
52036 \cdot 3 \\
52034 \cdot 5 \\
52033 \cdot 8 \\
52031 \cdot 1 \\
52024 \cdot 1 \\
52023 \cdot 5 \\
52022 \cdot 0 \\
52021 \cdot 1 \\
5200 \cdot 2 \\
5200.6 \\
51047 \cdot 1 \\
51037 \cdot 5\end{array}$ & 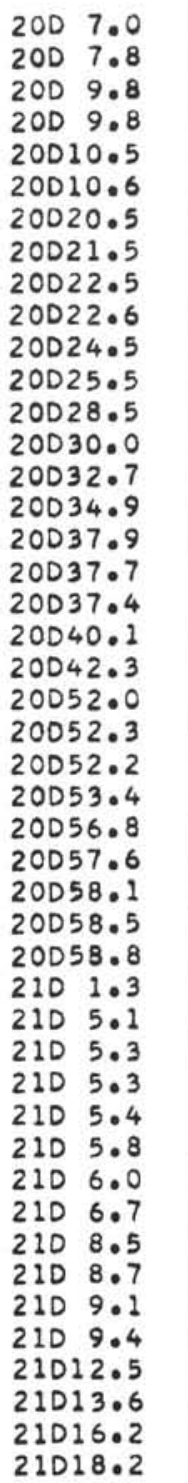 & 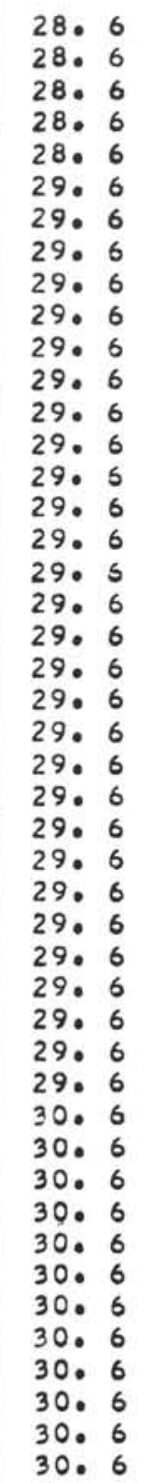 & $\begin{array}{r}18 H 58 \\
19 H 36 \\
19 H 37 \\
20 H 0 \\
2 O H \quad 2 \\
O H O \\
O H 24 \\
O H 24 \\
O H 56 \\
1 H 0 \\
2 H 12 \\
2 H 48 \\
5 H 0 \\
6 H 0 \\
7 H 0 \\
7 H 48 \\
8 H 42 \\
8 H 48 \\
8 H 52 \\
9 H 38 \\
10 H 18 \\
13 H 54 \\
14 H 0 \\
14 H 12 \\
14 H 35 \\
16 H 56 \\
17 H 35 \\
18 H 12 \\
18 H 44 \\
19 H 30 \\
20 H 58 \\
22 H 56 \\
23 H 20 \\
23 H 30 \\
23 H 35 \\
0 H 0 \\
0 H 10 \\
0 H 41 \\
1 H 55 \\
2 H 00 \\
2 H 15 \\
2 H 20 \\
3 H 42 \\
4 H 16 \\
5 H 30 \\
7 H \quad 4 \\
7 H 55\end{array}$ & $\begin{array}{l}57022 \cdot 0 \\
57015 \cdot 7 \\
57015 \cdot 5 \\
57011 \cdot 6 \\
57011 \cdot 3 \\
56031 \cdot 2 \\
56027 \cdot 2 \\
56021 \cdot 5 \\
56020 \cdot 8 \\
5608 \cdot 3 \\
5602 \cdot 1 \\
55038 \cdot 5 \\
55027 \cdot 8 \\
55016 \cdot 0 \\
5509 \cdot 3 \\
5500 \cdot 2 \\
54059 \cdot 1 \\
54058 \cdot 5 \\
54050 \cdot 8 \\
54044 \cdot 4 \\
5409 \cdot 2 \\
5408 \cdot 2 \\
5.406 \cdot 2 \\
5402 \cdot 5 \\
53039 \cdot 1 \\
53032 \cdot 7 \\
53026 \cdot 6 \\
53021 \cdot 1 \\
53013 \cdot 5 \\
52059 \cdot 3 \\
52038 \cdot 4 \\
52036 \cdot 6 \\
52036 \cdot 6 \\
52036 \cdot 3 \\
52034 \cdot 5 \\
52033 \cdot 8 \\
52031 \cdot 1 \\
52024 \cdot 1 \\
52023 \cdot 5 \\
52022 \cdot 0 \\
52021 \cdot 1 \\
5206 \cdot 2 \\
5200 \cdot 6 \\
51047 \cdot 1 \\
51037 \cdot 5 \\
51037 \cdot 4 \\
5503 \\
550\end{array}$ & 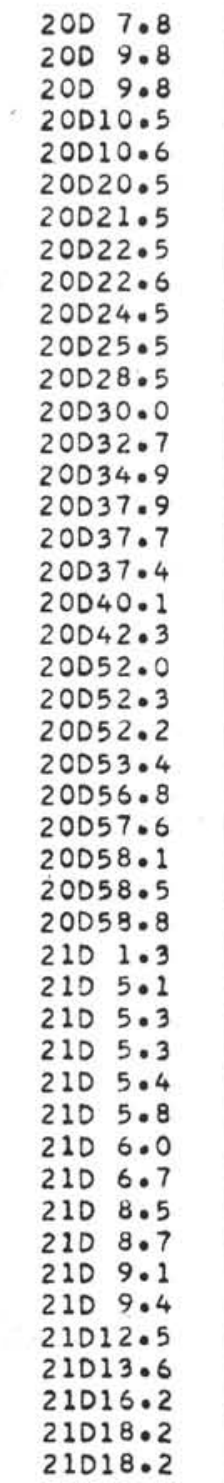 & $\begin{array}{r}1.54 \\
6.27 \\
0.16 \\
3.74 \\
0.32 \\
39.07 \\
3.94 \\
5.46 \\
0.66 \\
11.94 \\
5.93 \\
22.44 \\
10.14 \\
11.44 \\
6.71 \\
9.05 \\
1.02 \\
0.68 \\
7.70 \\
6.38 \\
34.52 \\
0.95 \\
1.89 \\
3.64 \\
22.29 \\
6.06 \\
5.75 \\
5.18 \\
7.12 \\
13.57 \\
19.95 \\
1.65 \\
0.03 \\
0.34 \\
1.72 \\
0.69 \\
2.58 \\
6.79 \\
0.58 \\
1.50 \\
0.87 \\
14.35 \\
5.36 \\
12.85 \\
9.28 \\
0.08\end{array}$ & 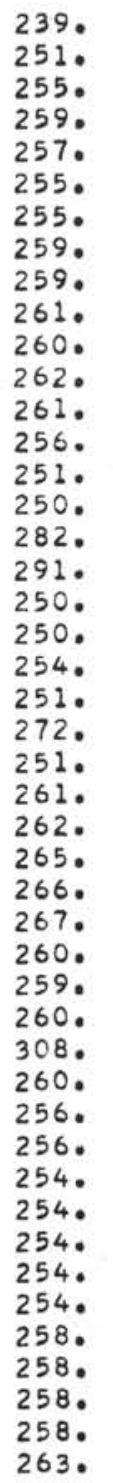 & $\begin{array}{r}11.591 \\
9.910 \\
9.751 \\
9.768 \\
9.738 \\
9.851 \\
9.853 \\
10.251 \\
9.932 \\
9.953 \\
9.883 \\
10.202 \\
10.149 \\
10.099 \\
10.065 \\
10.056 \\
10.256 \\
10.305 \\
10.052 \\
9.570 \\
9.591 \\
9.517 \\
9.477 \\
9.517 \\
9.488 \\
9.329 \\
9.330 \\
9.715 \\
9.295 \\
9.257 \\
10.144 \\
4.146 \\
0.222 \\
4.146 \\
4.135 \\
4.163 \\
5.010 \\
5.510 \\
7.010 \\
6.010 \\
10.510 \\
10.507 \\
9.467 \\
10.423 \\
5.923 \\
4.939\end{array}$ & $\begin{array}{r}1.54 \\
7.82 \\
7.98 \\
11.72 \\
12.05 \\
51.13 \\
55.07 \\
60.54 \\
61.20 \\
73.14 \\
79.07 \\
101.52 \\
111.67 \\
123.11 \\
129.82 \\
138.87 \\
139.90 \\
140.59 \\
148.29 \\
154.67 \\
189.20 \\
190.16 \\
192.05 \\
195.70 \\
218.00 \\
224.06 \\
229.82 \\
235.00 \\
242.12 \\
255.70 \\
275.65 \\
277.31 \\
277.35 \\
277.69 \\
279.42 \\
280.11 \\
282.70 \\
289.50 \\
290.08 \\
291.58 \\
292.46 \\
306.82 \\
312.18 \\
325.04 \\
334.32 \\
334.40\end{array}$ \\
\hline
\end{tabular}


TABLE 2

Glomar Challenger, DSDP Leg 25, Navigation Data

\begin{tabular}{|c|c|c|c|c|c|c|c|c|c|c|c|}
\hline $\begin{array}{c}\text { DATE } \\
\text { (day/mo) }\end{array}$ & $\begin{array}{c}\text { GMT } \\
(\mathrm{hr} / \mathrm{min})\end{array}$ & $\begin{array}{l}\text { LONGITUDE } \\
\text { (deg/min) }\end{array}$ & $\begin{array}{l}\text { LATITUDE } \\
\text { (deg/min) }\end{array}$ & $\begin{array}{c}\text { DATE } \\
\text { (day/mo) }\end{array}$ & $\underset{(\mathrm{hr} / \mathrm{min})}{\text { GMT }}$ & $\begin{array}{l}\text { LONGITUDE } \\
(\mathrm{deg} / \mathrm{min})\end{array}$ & $\begin{array}{l}\text { LATITUDE } \\
\text { (deg/min) }\end{array}$ & $\begin{array}{l}\text { DIST } \\
(\mathrm{nmi})\end{array}$ & $\begin{array}{l}\text { CSE } \\
\left({ }^{\circ}\right)\end{array}$ & $\begin{array}{l}\text { SPEED } \\
(k t)\end{array}$ & $\begin{array}{l}\text { DISTT } \\
\text { (nmi) }\end{array}$ \\
\hline 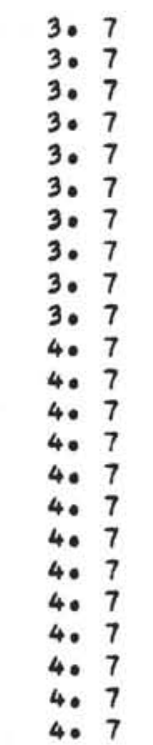 & $\begin{array}{l}12 \mathrm{H} 23 \\
12 \mathrm{H} 3 \mathrm{O} \\
13 \mathrm{H} 50 \\
14 \mathrm{HO} 0 \\
14 \mathrm{H} 52 \\
16 \mathrm{H} 46 \\
17 \mathrm{H} 20 \\
18 \mathrm{H} 32 \\
19 \mathrm{H} 34 \\
21 \mathrm{H} 16 \\
\mathrm{OH} 0 \\
1 \mathrm{H} 12 \\
1 \mathrm{H} 49 \\
2 \mathrm{H} 18 \\
7 \mathrm{H} 2 \mathrm{O} \\
7 \mathrm{H} 45 \\
8 \mathrm{H} 40 \\
9 \mathrm{H} 6 \\
9 \mathrm{H} 42 \\
12 \mathrm{H} 18 \\
12 \mathrm{H} 52 \\
14 \mathrm{H} 2 \\
2 \mathrm{OH} 28\end{array}$ & $\begin{array}{l}51040.7 \\
51041.0 \\
51046.3 \\
51046.8 \\
51052.0 \\
5201.9 \\
5204.7 \\
52010.4 \\
52015.4 \\
52024.2 \\
52038.1 \\
52044.2 \\
52046.8 \\
52048.7 \\
5309.9 \\
53011.4 \\
53015.1 \\
53017.1 \\
53019.5 \\
53031.1 \\
53033.7 \\
53038.1 \\
540 \quad 6.6\end{array}$ & $\begin{array}{l}21017 \cdot 7 \\
21016 \cdot 9 \\
2108 \cdot 9 \\
2108 \cdot 1 \\
2100.8 \\
20045.1 \\
20040.4 \\
20030.4 \\
20022.0 \\
2008.7 \\
19047.7 \\
19038.6 \\
19033.5 \\
19029.5 \\
18049.0 \\
18045.8 \\
18039 \cdot 1 \\
18036.1 \\
18031.7 \\
18013.9 \\
18010.1 \\
1802.0 \\
17015.9\end{array}$ & 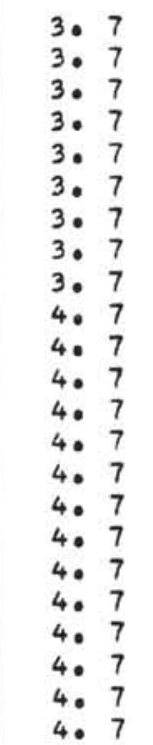 & $\begin{array}{l}12 \mathrm{H} 30 \\
13 \mathrm{H} 50 \\
14 \mathrm{H} 0 \\
14 \mathrm{H} 52 \\
16 \mathrm{H} 46 \\
17 \mathrm{H} 20 \\
18 \mathrm{H} 32 \\
19 \mathrm{H} 34 \\
21 \mathrm{H} 16 \\
0 \mathrm{H} 0 \\
1 \mathrm{H} 12 \\
1 \mathrm{H} 49 \\
2 \mathrm{H} 18 \\
7 \mathrm{H} 20 \\
7 \mathrm{H} 45 \\
8 \mathrm{H} 40 \\
9 \mathrm{H} 6 \\
9 \mathrm{H} 42 \\
12 \mathrm{H} 18 \\
12 \mathrm{H} 52 \\
14 \mathrm{H} 2 \\
20 \mathrm{H} 28 \\
23 \mathrm{H} 30\end{array}$ & $\begin{array}{l}51041 \cdot 0 \\
51046 \cdot 3 \\
51046 \cdot 8 \\
51052 \cdot 0 \\
5201 \cdot 9 \\
5204 \cdot 7 \\
52010 \cdot 4 \\
52015 \cdot 4 \\
52024 \cdot 2 \\
52038 \cdot 1 \\
52044 \cdot 2 \\
52046 \cdot 8 \\
52048 \cdot 7 \\
5309 \cdot 9 \\
53011 \cdot 4 \\
53015 \cdot 1 \\
53017 \cdot 1 \\
53019 \cdot 5 \\
53031 \cdot 1 \\
53033 \cdot 7 \\
53038 \cdot 1 \\
5406 \cdot 6 \\
54021 \cdot 4\end{array}$ & $\begin{array}{l}21016.9 \\
2108.9 \\
2108.1 \\
2100.8 \\
20045.1 \\
20040.4 \\
20030.4 \\
20022.0 \\
2008.7 \\
19047.7 \\
19038.6 \\
19033.5 \\
19029.5 \\
18049.0 \\
18045.8 \\
18039.1 \\
18036.1 \\
18031.7 \\
18013.9 \\
18010.1 \\
1802.0 \\
17015.9 \\
16055.1\end{array}$ & $\begin{array}{r}0.83 \\
9.41 \\
0.93 \\
8.77 \\
18.24 \\
5.35 \\
11.38 \\
9.63 \\
15.67 \\
24.71 \\
10.83 \\
5.61 \\
4.43 \\
45.23 \\
3.47 \\
7.58 \\
3.56 \\
4.90 \\
21.01 \\
4.54 \\
9.12 \\
53.58 \\
25.18\end{array}$ & $\begin{array}{l}24 \circ^{\circ} \\
310^{\circ} \\
330^{\circ} \\
330^{\circ} \\
310^{\circ} \\
300^{\circ} \\
280^{\circ} \\
290^{\circ} \\
320^{\circ} \\
320^{\circ} \\
320^{\circ} \\
260^{\circ} \\
230^{\circ} \\
260^{\circ} \\
250^{\circ} \\
280^{\circ} \\
310^{\circ} \\
280^{\circ} \\
320^{\circ} \\
330^{\circ} \\
270^{\circ} \\
30^{\circ} \\
340^{\circ}\end{array}$ & $\begin{array}{r}7.170 \\
7.062 \\
5.608 \\
10.122 \\
9.605 \\
9.449 \\
9.486 \\
9.321 \\
9.223 \\
9.042 \\
9.030 \\
9.109 \\
9.178 \\
8.986 \\
8.334 \\
8.272 \\
8.231 \\
8.175 \\
8.082 \\
8.012 \\
7.823 \\
8.330 \\
8.302\end{array}$ & $\begin{array}{r}0.83 \\
10.25 \\
11.18 \\
19.96 \\
38.21 \\
43.56 \\
54.94 \\
64.58 \\
80.26 \\
104.97 \\
115.81 \\
121.43 \\
125.86 \\
171.10 \\
174.57 \\
182.15 \\
185.72 \\
190.63 \\
211.64 \\
216.18 \\
225.31 \\
278.90 \\
304.08\end{array}$ \\
\hline
\end{tabular}


TABLE 3

Glomar Challenger, DSDP Leg 25, Navigation Data

\begin{tabular}{|c|c|c|c|c|c|c|c|c|c|c|c|}
\hline $\begin{array}{c}\text { DATE } \\
\text { (day/mo) }\end{array}$ & $\underset{(\mathrm{hr} / \mathrm{min})}{\mathrm{GMT}}$ & $\begin{array}{l}\text { LONGITUDE } \\
(\mathrm{deg} / \mathrm{min})\end{array}$ & $\begin{array}{l}\text { LATITUDE } \\
\text { (deg/min) }\end{array}$ & $\begin{array}{c}\text { DATE } \\
\text { (day/mo) }\end{array}$ & $\begin{array}{c}\text { GMT } \\
(\mathrm{hr} / \mathrm{min})\end{array}$ & $\begin{array}{l}\text { LONGITUDE } \\
\text { (deg/min) }\end{array}$ & $\begin{array}{l}\text { LATITUDE } \\
\text { (deg/min) }\end{array}$ & $\begin{array}{c}\text { DIST } \\
(\mathrm{nmi})\end{array}$ & $\begin{array}{l}\text { CSE } \\
\left({ }^{\circ}\right)\end{array}$ & $\begin{array}{l}\text { SPEED } \\
(k t)\end{array}$ & $\begin{array}{l}\text { DISTT } \\
(\mathrm{nmi})\end{array}$ \\
\hline 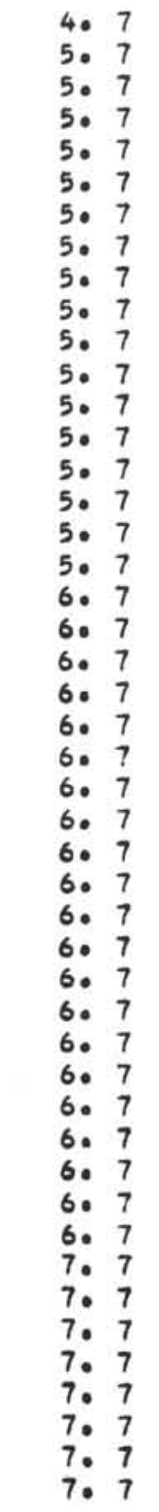 & $\begin{array}{r}23 H 30 \\
O H O \\
O H 14 \\
1 H 30 \\
1 H 30 \\
2 H 0 \\
2 H 44 \\
6 H 21 \\
7 H 38 \\
8 H 16 \\
11 H 58 \\
12 H 22 \\
13 H 14 \\
16 H 54 \\
17 H 31 \\
18 H 42 \\
19 H 40 \\
21 H 30 \\
23 H 30 \\
0 H 0 \\
O H 338 \\
2 H 0 \\
2 H 26 \\
2 H 26 \\
2 H 54 \\
4 H 32 \\
6 H 16 \\
7 H 22 \\
8 H 30 \\
8 H 47 \\
9 H 14 \\
9 H 28 \\
9 H 36 \\
12 H 22 \\
14 H 10 \\
16 H 8 \\
17 H 54 \\
18 H 52 \\
19 H 18 \\
20 H 40 \\
21 H 25 \\
0 H 00 \\
0 H 14 \\
1 H 36 \\
2 H 4 \\
3 H 54 \\
5 H 40 \\
7 H 199 \\
7 H 21\end{array}$ & 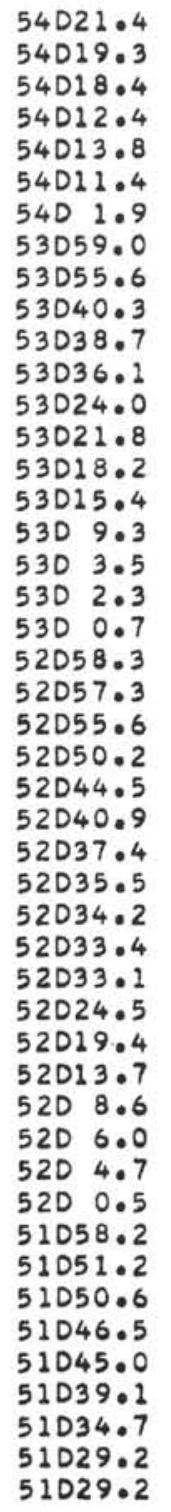 & $\begin{array}{l}16055 \cdot 1 \\
16050.7 \\
16048.7 \\
16037.9 \\
16033.2 \\
16026.5 \\
15053.5 \\
15046.6 \\
15037.0 \\
1504.9 \\
1501.7 \\
14054.8 \\
14022.5 \\
14016.9 \\
1406.3 \\
13058.0 \\
13041.9 \\
13024.4 \\
13020.0 \\
13014.5 \\
1303.2 \\
12059.7 \\
12055.6 \\
12041.6 \\
12026.8 \\
12017.0 \\
1206.8 \\
1204.9 \\
1200.9 \\
11058.8 \\
11058.2 \\
11033.5 \\
11017.1 \\
10059.6 \\
10044.0 \\
10035.7 \\
10032.0 \\
10020.5 \\
10014.4 \\
9053.3 \\
9051.4 \\
9040.6 \\
9036.4 \\
9020.4 \\
906.1 \\
8051.1 \\
8050.8\end{array}$ & 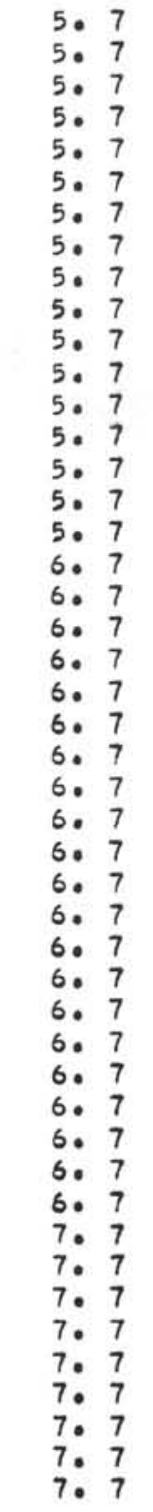 & 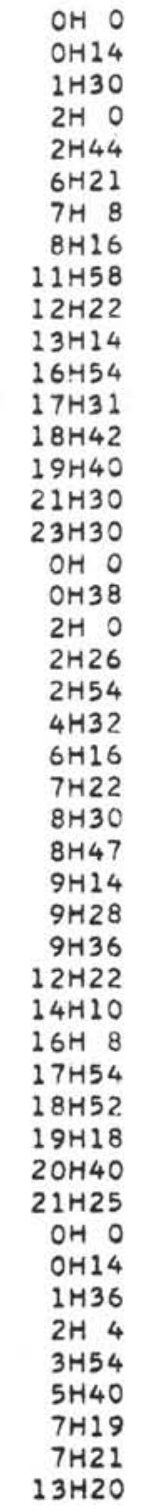 & 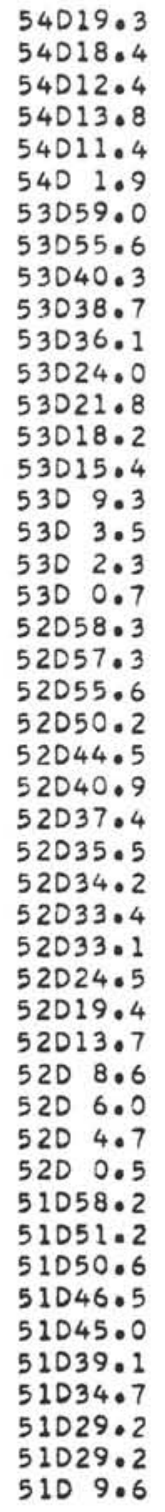 & 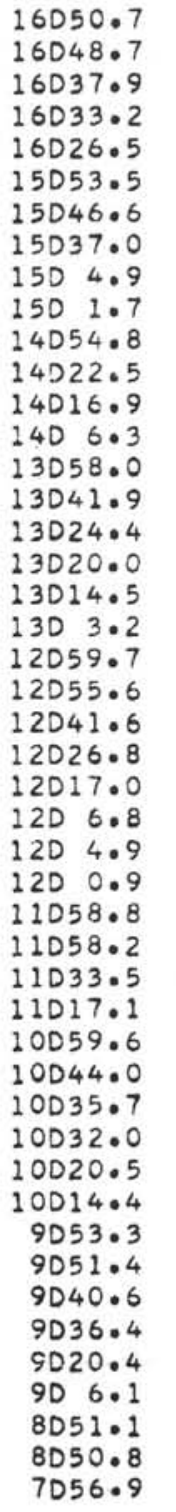 & $\begin{array}{r}4.85 \\
2.21 \\
12.25 \\
4.89 \\
6.98 \\
34.31 \\
7.50 \\
10.14 \\
35.36 \\
3.46 \\
7.43 \\
34.38 \\
5.89 \\
11.26 \\
8.73 \\
17.16 \\
18.30 \\
4.55 \\
5.80 \\
11.51 \\
3.66 \\
4.42 \\
14.96 \\
15.82 \\
10.41 \\
10.65 \\
2.75 \\
4.21 \\
2.20 \\
0.66 \\
26.15 \\
17.15 \\
18.38 \\
16.39 \\
8.68 \\
3.88 \\
12.26 \\
6.47 \\
22.23 \\
1.99 \\
11.54 \\
4.45 \\
17.03 \\
14.95 \\
15.85 \\
0.30 \\
57.43\end{array}$ & 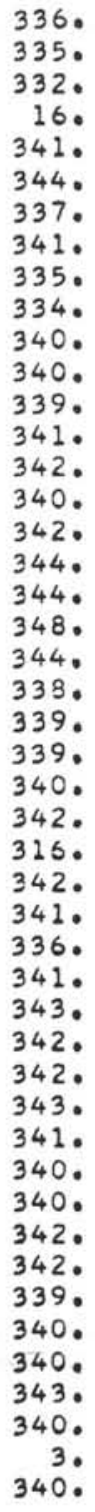 & $\begin{array}{l}9.707 \\
9.492 \\
9.671 \\
9.780 \\
9.518 \\
9.487 \\
9.577 \\
8.955 \\
9.558 \\
8.674 \\
8.578 \\
9.376 \\
9.562 \\
9.519 \\
9.039 \\
9.364 \\
9.153 \\
9.119 \\
9.159 \\
8.425 \\
8.456 \\
9.484 \\
9.164 \\
9.129 \\
9.472 \\
9.401 \\
9.722 \\
9.377 \\
9.449 \\
4.983 \\
9.452 \\
9.530 \\
9.347 \\
9.279 \\
8.988 \\
8.956 \\
8.974 \\
8.638 \\
8.607 \\
8.567 \\
8.444 \\
9.548 \\
9.293 \\
8.464 \\
9.611 \\
9.147 \\
9.599\end{array}$ & $\begin{array}{r}4.85 \\
7.06 \\
19.31 \\
24 \cdot 20 \\
31.18 \\
65.50 \\
73.00 \\
83.15 \\
118.51 \\
121.98 \\
129.42 \\
163.80 \\
169.70 \\
180.96 \\
189.70 \\
206.87 \\
225.18 \\
229.74 \\
235.54 \\
247.05 \\
250.72 \\
255.14 \\
270.11 \\
285.94 \\
296.36 \\
307.01 \\
309.77 \\
313.99 \\
316.19 \\
316.85 \\
343.01 \\
360.16 \\
378.54 \\
394.94 \\
403.63 \\
407.51 \\
419.77 \\
426.25 \\
448.49 \\
450.49 \\
462.03 \\
466.48 \\
483.52 \\
498.48 \\
514 \cdot 33 \\
514.64 \\
572.07\end{array}$ \\
\hline
\end{tabular}


TABLE 3 - Continued

\begin{tabular}{|c|c|c|c|c|c|c|c|c|c|c|c|}
\hline $\begin{array}{c}\text { DATE } \\
\text { (day/mo) }\end{array}$ & $\begin{array}{c}\text { GMT } \\
(\mathrm{hr} / \mathrm{min})\end{array}$ & $\begin{array}{l}\text { LONGITUDE } \\
\text { (deg/min) }\end{array}$ & $\begin{array}{l}\text { LATITUDE } \\
(\mathrm{deg} / \mathrm{min})\end{array}$ & $\begin{array}{c}\text { DATE } \\
\text { (day/mo) }\end{array}$ & $\begin{array}{c}\text { GMT } \\
(\mathrm{hr} / \mathrm{min})\end{array}$ & $\begin{array}{l}\text { LONGITUDE } \\
\text { (deg/min) }\end{array}$ & $\begin{array}{l}\text { LATITUDE } \\
\text { (deg/min) }\end{array}$ & $\begin{array}{c}\text { DIST } \\
(\mathrm{nmi})\end{array}$ & $\begin{array}{l}\text { CSE } \\
\left({ }^{\circ}\right)\end{array}$ & $\begin{array}{l}\text { SPEED } \\
(k t)\end{array}$ & $\begin{array}{l}\text { DISTT } \\
(\mathrm{nmi})\end{array}$ \\
\hline $\begin{array}{l}7: 7 \\
7: 7 \\
707 \\
707 \\
707 \\
707 \\
807 \\
807 \\
807 \\
807 \\
807 \\
807 \\
807 \\
807 \\
807 \\
807 \\
807 \\
807 \\
807 \\
807 \\
807 \\
807 \\
807 \\
807 \\
807\end{array}$ & $\begin{array}{l}13 H 2 O \\
17 H 16 \\
17 H 40 \\
18 H 54 \\
19 H 54 \\
20 H 30 \\
O H \quad 0 \\
O H 50 \\
1 H 25 \\
2 H 36 \\
4 H 36 \\
6 H 24 \\
7 H 32 \\
9 H 18 \\
12 H 32 \\
13 H 0 \\
14 H 16 \\
14 H 49 \\
15 H 37 \\
16 H 16 \\
18 H 4 \\
19 H 0 \\
19 H 4 \\
20 H 48 \\
21 H 27\end{array}$ & $\begin{array}{l}5109.6 \\
50059.1 \\
50058.1 \\
50054.7 \\
50051.8 \\
50049.7 \\
50035.8 \\
50032.5 \\
50030.2 \\
50026.5 \\
50020.3 \\
50014.8 \\
50011.6 \\
5007.1 \\
5001.3 \\
5000.5 \\
49057.1 \\
49055.8 \\
49052.9 \\
49050.4 \\
49042.9 \\
49039.1 \\
49039.0 \\
49031.8 \\
49028.9\end{array}$ & $\begin{array}{l}7056.9 \\
7021.6 \\
7017.8 \\
706.3 \\
6 D 57.4 \\
6052.2 \\
6022.5 \\
6015 \cdot 5 \\
6010.4 \\
5059.9 \\
5042.5 \\
5027.0 \\
5017 \cdot 0 \\
500.7 \\
4030.6 \\
4025.8 \\
4013.6 \\
408.5 \\
401.5 \\
3056.1 \\
3041.4 \\
3033.1 \\
3032.7 \\
3023.6 \\
3021.2\end{array}$ & 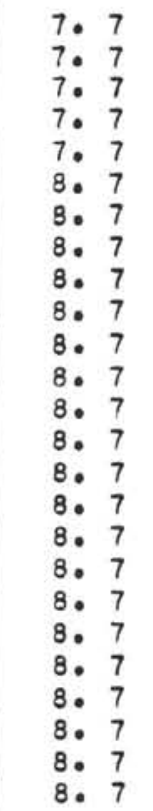 & $\begin{array}{r}17 H 16 \\
17 H 40 \\
18 H 54 \\
19 H 54 \\
20 H 30 \\
0 H 0 \\
0 H 50 \\
1 H 25 \\
2 H 36 \\
4 H 36 \\
6 H 24 \\
7 H 32 \\
9 H 18 \\
12 H 32 \\
13 H 0 \\
14 H 16 \\
14 H 49 \\
15 H 37 \\
16 H 16 \\
18 H 4 \\
19 H 0 \\
19 H 44 \\
20 H 48 \\
21 H 27 \\
21 H 48\end{array}$ & $\begin{array}{l}50059 \cdot 1 \\
50058 \cdot 1 \\
50054 \cdot 7 \\
50051 \cdot 8 \\
50049 \cdot 7 \\
50035 \cdot 8 \\
50032 \cdot 5 \\
50030 \cdot 2 \\
50026 \cdot 5 \\
50020 \cdot 3 \\
50014 \cdot 8 \\
50011 \cdot 6 \\
5007 \cdot 1 \\
5001 \cdot 3 \\
5000.5 \\
49057 \cdot 1 \\
49055 \cdot 8 \\
49052 \cdot 9 \\
49050.4 \\
49042.9 \\
49039 \cdot 1 \\
49039 \cdot 0 \\
49031.3 \\
49028.9 \\
49027 \cdot 5\end{array}$ & 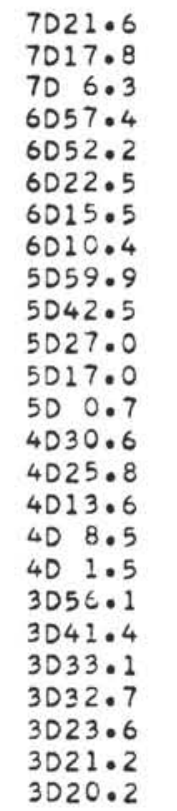 & $\begin{array}{r}36.82 \\
3.89 \\
12.02 \\
9.35 \\
5.58 \\
32.75 \\
7.79 \\
5.54 \\
11.18 \\
18.47 \\
16.45 \\
10.50 \\
16.91 \\
30.65 \\
4.76 \\
12.77 \\
5.25 \\
7.50 \\
6.03 \\
16.51 \\
9.11 \\
0.43 \\
11.52 \\
3.72 \\
1.67\end{array}$ & 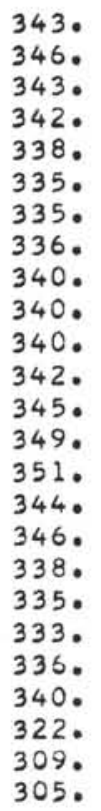 & $\begin{array}{r}9.361 \\
9.742 \\
9.750 \\
9.359 \\
9.306 \\
9.359 \\
9.355 \\
9.504 \\
9.448 \\
9.237 \\
9.139 \\
9.266 \\
9.573 \\
9.481 \\
10.202 \\
10.086 \\
9.563 \\
9.385 \\
9.284 \\
9.176 \\
9.761 \\
6.480 \\
6.707 \\
5.738 \\
4.796\end{array}$ & $\begin{array}{l}608.89 \\
612.79 \\
624.82 \\
634.18 \\
639.76 \\
672.52 \\
680.31 \\
685.86 \\
697.04 \\
715.51 \\
731.96 \\
742.47 \\
759.38 \\
790.04 \\
794.80 \\
807.57 \\
812.83 \\
820.34 \\
826.38 \\
842.90 \\
852.01 \\
852.44 \\
864.06 \\
867.79 \\
869.47\end{array}$ \\
\hline
\end{tabular}


TABLE 4

Glomar Challenger, DSDP Leg 25, Navigation Data

\begin{tabular}{|c|c|c|c|c|c|c|c|c|c|c|c|}
\hline $\begin{array}{c}\text { DATE } \\
\text { (day/mo) }\end{array}$ & $\underset{(\mathrm{hr} / \mathrm{min})}{\mathrm{GMT}}$ & $\begin{array}{l}\text { LONGITUDE } \\
\text { (deg/min) }\end{array}$ & $\begin{array}{l}\text { LATITUDE } \\
\text { (deg/min) }\end{array}$ & $\begin{array}{c}\text { DATE } \\
\text { (day/mo) }\end{array}$ & $\begin{array}{c}\text { GMT } \\
(\mathrm{hr} / \mathrm{min})\end{array}$ & $\begin{array}{l}\text { LONGITUDE } \\
\text { (deg/min) }\end{array}$ & $\begin{array}{l}\text { LATITUDE } \\
\text { (deg/min) }\end{array}$ & $\begin{array}{r}\text { DIST } \\
(\mathrm{nmi})\end{array}$ & $\begin{array}{l}\text { CSE } \\
\left({ }^{\circ}\right)\end{array}$ & $\begin{array}{l}\text { SPEED } \\
(k t)\end{array}$ & $\begin{array}{l}\text { DISTT } \\
(\mathrm{nmi})\end{array}$ \\
\hline $\begin{array}{l}1207 \\
1207 \\
1207 \\
1207 \\
1207 \\
1207 \\
1207 \\
1207 \\
1207 \\
1207 \\
1207 \\
1207 \\
1207 \\
1207 \\
1207 \\
1207 \\
1207 \\
1207 \\
1207 \\
1207 \\
1307 \\
1307 \\
1307 \\
1307 \\
1307 \\
1307 \\
1307 \\
1307 \\
1307 \\
1307 \\
1307 \\
1307 \\
1307 \\
1307 \\
1307 \\
1307 \\
1307 \\
1307 \\
1307 \\
1307 \\
1307\end{array}$ & $\begin{array}{l}2 H 22 \\
2 H 49 \\
3 H 0 \\
4 H 44 \\
5 H 6 \\
6 H 32 \\
6 H 40 \\
6 H 43 \\
7 H 48 \\
8 H 20 \\
12 H \quad 4 \\
12 H 34 \\
16 H 26 \\
16 H 46 \\
18 H 10 \\
18 H 43 \\
19 H 20 \\
20 H 30 \\
21 H 06 \\
22 H 0 \\
0 H 0 \\
0 H 44 \\
0 H 25 \\
1 H 0 \\
1 H 50 \\
2 H 14 \\
2 H 41 \\
4 H 20 \\
4 H 50 \\
5 H 42 \\
6 H 4 \\
6 H 58 \\
8 H 44 \\
10 H 0 \\
11 H 44 \\
12 H 2 \\
12 H 3 \\
13 H 26 \\
13 H 40 \\
13 H 45 \\
13 H 52\end{array}$ & 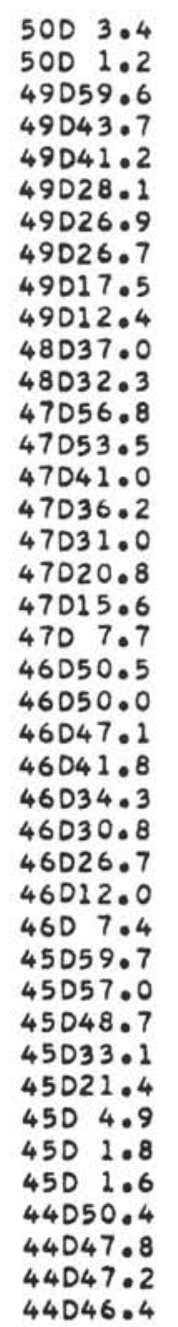 & 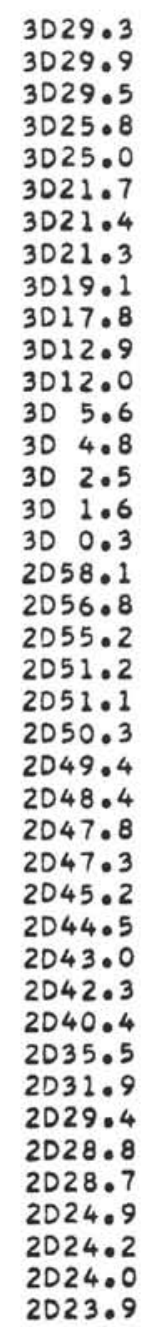 & 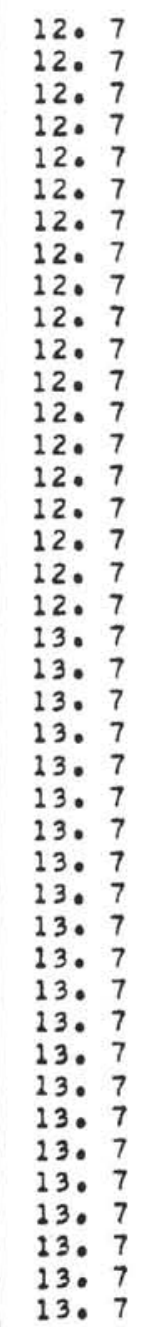 & $\begin{array}{l}2 H 49 \\
3 H 0 \\
4 H 44 \\
5 H 6 \\
6 H 32 \\
6 H 40 \\
6 H 43 \\
7 H 48 \\
8 H 20 \\
12 H \quad 4 \\
12 H 34 \\
16 H 26 \\
16 H 46 \\
18 H 10 \\
18 H 43 \\
19 H 2 O \\
20 H 30 \\
21 H 6 \\
22 H 0 \\
0 H 0 \\
0 H \quad 4 \\
0 H 25 \\
1 H O \\
1 H 50 \\
2 H 14 \\
2 H 41 \\
4 H 20 \\
4 H 50 \\
5 H 42 \\
6 H 4 \\
6 H 58 \\
8 H 44 \\
10 H 0 \\
11 H 44 \\
12 H \quad 2 \\
12 H \quad 3 \\
13 H 26 \\
13 H 40 \\
13 H 45 \\
13 H 52 \\
14 H 40\end{array}$ & $\begin{array}{l}5001 \cdot 2 \\
49059 \cdot 6 \\
49043 \cdot 7 \\
49041 \cdot 2 \\
49028 \cdot 1 \\
49026 \cdot 9 \\
49026 \cdot 7 \\
49017 \cdot 5 \\
49012 \cdot 4 \\
48037 \cdot 0 \\
48032 \cdot 3 \\
47056 \cdot 8 \\
47053 \cdot 5 \\
47041 \cdot 0 \\
47036 \cdot 2 \\
47031 \cdot 0 \\
47020 \cdot 8 \\
47015 \cdot 6 \\
4707 \cdot 7 \\
46050 \cdot 5 \\
46050 \cdot 0 \\
46047 \cdot 1 \\
46041 \cdot 8 \\
46034 \cdot 3 \\
46030 \cdot 8 \\
46026 \cdot 7 \\
46012 \cdot 0 \\
4607.4 \\
45059 \cdot 7 \\
45057 \cdot 0 \\
45048 \cdot 7 \\
45033 \cdot 1 \\
45021 \cdot 4 \\
4504 \cdot 9 \\
4501 \cdot 8 \\
4501.6 \\
44050 \cdot 4 \\
44047 \cdot 8 \\
44047 \cdot 2 \\
44 D 46 \cdot 4 \\
44040 \cdot 8\end{array}$ & 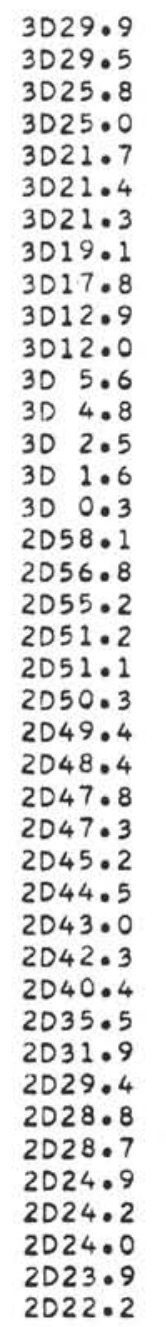 & $\begin{array}{r}2.26 \\
1.64 \\
16.46 \\
2.53 \\
13.57 \\
1.17 \\
0.21 \\
9.56 \\
5.19 \\
35.98 \\
4.83 \\
36.25 \\
3.41 \\
12.77 \\
4.83 \\
5.45 \\
10.47 \\
5.40 \\
7.99 \\
17.77 \\
0.59 \\
3.02 \\
5.35 \\
7.65 \\
3.557 \\
4.009 \\
14.98 \\
4.60 \\
7.95 \\
2.80 \\
8.45 \\
16.54 \\
12.21 \\
16.85 \\
3.17 \\
0.14 \\
11.96 \\
2.60 \\
0.64 \\
0.89 \\
5.88\end{array}$ & 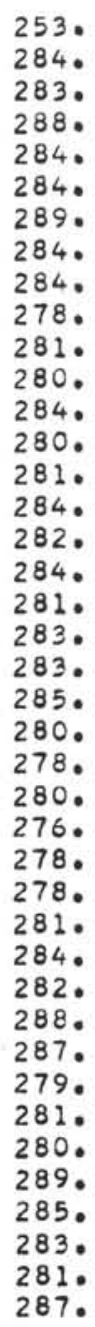 & $\begin{array}{r}5.037 \\
8.996 \\
9.498 \\
7.190 \\
9.469 \\
8.840 \\
4.384 \\
8.833 \\
9.739 \\
9.638 \\
9.663 \\
9.376 \\
10.237 \\
9.125 \\
8.793 \\
8.852 \\
8.976 \\
9.006 \\
8.883 \\
8.889 \\
8.898 \\
8.639 \\
9.176 \\
9.191 \\
8.925 \\
9.110 \\
9.081 \\
9.217 \\
9.183 \\
7.647 \\
9.397 \\
9.364 \\
9.645 \\
9.726 \\
10.584 \\
8.684 \\
8.651 \\
11.174 \\
7.692 \\
7.707 \\
7.354\end{array}$ & $\begin{array}{r}2.26 \\
3.91 \\
20.37 \\
23.01 \\
36.58 \\
37.76 \\
37.98 \\
47.55 \\
52.75 \\
88.73 \\
93.56 \\
129.82 \\
133.23 \\
146.00 \\
150.84 \\
156.30 \\
166.77 \\
172.18 \\
180.17 \\
197.95 \\
198.54 \\
201.57 \\
206.92 \\
214.58 \\
218.15 \\
222.25 \\
237.23 \\
241.84 \\
249.80 \\
252.60 \\
261.06 \\
277.61 \\
289.82 \\
306.68 \\
309.86 \\
310.00 \\
321.97 \\
324.58 \\
325.22 \\
326.12 \\
332.00\end{array}$ \\
\hline
\end{tabular}


TABLE 5

Glomar Challenger, DSDP Leg 25, Navigation Data

\begin{tabular}{|c|c|c|c|c|c|c|c|c|c|c|c|}
\hline $\begin{array}{c}\text { DATE } \\
\text { (day/mo) }\end{array}$ & $\begin{array}{c}\text { GMT } \\
(\mathrm{hr} / \mathrm{min})\end{array}$ & $\begin{array}{l}\text { LONGITUDE } \\
\text { (deg/min) }\end{array}$ & $\begin{array}{l}\text { LATITUDE } \\
\text { (deg/min) }\end{array}$ & $\begin{array}{c}\text { DATE } \\
(\text { day/mo })\end{array}$ & $\begin{array}{c}\text { GMT } \\
(h r / \min )\end{array}$ & $\begin{array}{l}\text { LONGITUDE } \\
\text { (deg/min) }\end{array}$ & $\begin{array}{l}\text { LATITUDE } \\
\text { (deg/min) }\end{array}$ & $\begin{array}{c}\text { DIST } \\
(\mathrm{nmi})\end{array}$ & $\begin{array}{l}\text { CSE } \\
\left({ }^{\circ}\right)\end{array}$ & $\begin{array}{l}\text { SPEED } \\
(k t)\end{array}$ & $\begin{array}{l}\text { DISTT } \\
(n m i)\end{array}$ \\
\hline $\begin{array}{l}18 \cdot 7 \\
18: 7\end{array}$ & $\begin{array}{l}11 H 8 \\
12 H 24\end{array}$ & $\begin{array}{l}44040.8 \\
44035.6\end{array}$ & $\begin{array}{l}2022.2 \\
2029.1\end{array}$ & $\begin{array}{l}18.7 \\
18 \cdot 7\end{array}$ & $\begin{array}{l}12 \mathrm{H} 24 \\
12 \mathrm{H} 36\end{array}$ & $\begin{array}{l}44035.6 \\
44034.8\end{array}$ & $\begin{array}{l}2029.1 \\
2030.1\end{array}$ & $\begin{array}{l}8.65 \\
1.27\end{array}$ & $\begin{array}{l}217 \\
218\end{array}$ & $\begin{array}{l}6.835 \\
6.370\end{array}$ & $\begin{array}{l}8.65 \\
9.93\end{array}$ \\
\hline
\end{tabular}

TABLE 6

Glomar Challenger, DSDP Leg 25, Navigation Data

\begin{tabular}{|c|c|c|c|c|c|c|c|c|c|c|c|}
\hline $\begin{array}{c}\text { DATE } \\
\text { (day/mo) }\end{array}$ & $\underset{(h r / m i n)}{\text { GMT }}$ & $\begin{array}{l}\text { LONGITUDE } \\
(\mathrm{deg} / \mathrm{min})\end{array}$ & $\begin{array}{l}\text { LATITUDE } \\
\text { (deg/min) }\end{array}$ & $\begin{array}{c}\text { DATE } \\
(\text { day } / \mathrm{mo})\end{array}$ & $\begin{array}{c}\text { GMT } \\
(\mathrm{hr} / \mathrm{min})\end{array}$ & $\begin{array}{l}\text { LONGITUDE } \\
(\mathrm{deg} / \mathrm{min})\end{array}$ & $\begin{array}{l}\text { LATITUDE } \\
\text { (deg/min) }\end{array}$ & $\begin{array}{l}\text { DIST } \\
(\mathrm{nmi})\end{array}$ & $\begin{array}{l}\text { CSE } \\
\left({ }^{\circ}\right)\end{array}$ & $\begin{array}{l}\text { SPEED } \\
(k t)\end{array}$ & $\begin{array}{l}\text { DISTT } \\
(n m i)\end{array}$ \\
\hline $\begin{array}{l}18 \cdot 7 \\
18.7 \\
18.7\end{array}$ & $\begin{array}{l}12 \mathrm{H} 36 \\
12 \mathrm{H} 42 \\
14 \mathrm{H} 12\end{array}$ & $\begin{array}{l}44034.8 \\
44035.1 \\
44040.3\end{array}$ & $\begin{array}{l}2030.1 \\
2030.7 \\
2040.6\end{array}$ & $\begin{array}{l}18 \cdot 7 \\
18 \cdot 7 \\
18 \cdot 7\end{array}$ & $\begin{array}{l}12 \mathrm{H} 42 \\
14 \mathrm{H} 12 \\
14 \mathrm{H} 47\end{array}$ & $\begin{array}{l}44035 \cdot 1 \\
44040.3 \\
44042 \cdot 2\end{array}$ & $\begin{array}{l}2030.7 \\
2040.6 \\
2044.4\end{array}$ & $\begin{array}{r}0.73 \\
11.11 \\
4.29\end{array}$ & $\begin{array}{l}157^{\circ} \\
152^{\circ} \\
153^{\circ}\end{array}$ & $\begin{array}{l}7.376 \\
7.412 \\
7.369\end{array}$ & $\begin{array}{r}0.73 \\
11.85 \\
16.15\end{array}$ \\
\hline
\end{tabular}


TABLE 7

Glomar Challenger, DSDP Leg 25, Navigation Data

\begin{tabular}{|c|c|c|c|c|c|c|c|c|c|c|c|}
\hline $\begin{array}{c}\text { DATE } \\
\text { (day/mo) }\end{array}$ & $\begin{array}{c}\text { GMT } \\
(\mathrm{hr} / \mathrm{min})\end{array}$ & $\begin{array}{l}\text { LONGITUDE } \\
(\mathrm{deg} / \mathrm{min})\end{array}$ & $\begin{array}{l}\text { LATITUDE } \\
(\mathrm{deg} / \mathrm{min})\end{array}$ & $\begin{array}{c}\text { DATE } \\
(\text { day/mo })\end{array}$ & $\begin{array}{c}\text { GMT } \\
(\mathrm{hr} / \mathrm{min})\end{array}$ & $\begin{array}{l}\text { LONGITUDE } \\
\text { (deg/min) }\end{array}$ & $\begin{array}{l}\text { LATITUDE } \\
\text { (deg/min) }\end{array}$ & $\begin{array}{l}\text { DIST } \\
(\mathrm{nmi})\end{array}$ & $\begin{array}{l}\text { CSE } \\
\left({ }^{\circ}\right)\end{array}$ & $\begin{array}{l}\text { SPEED } \\
(k t)\end{array}$ & $\begin{array}{l}\text { DISTT } \\
(\mathrm{nmi})\end{array}$ \\
\hline 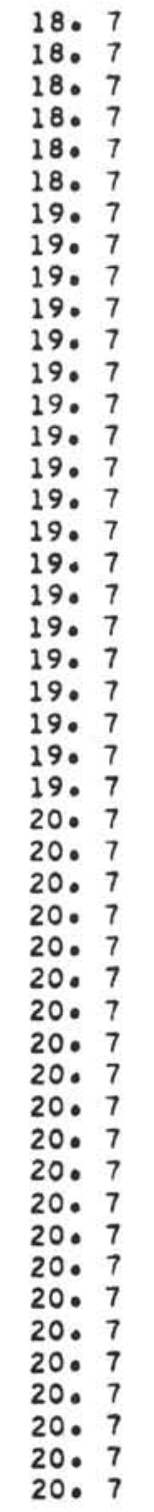 & $\begin{array}{l}14 H 47 \\
17 H 10 \\
21 H 36 \\
22 H 36 \\
23 H 36 \\
23 H 54 \\
0 H 0 \\
0 H 48 \\
1 H 44 \\
2 H 38 \\
3 H 0 \\
4 H 8 \\
4 H 54 \\
5 H 50 \\
7 H 26 \\
12 H 30 \\
13 H 0 \\
13 H 14 \\
14 H 16 \\
14 H 45 \\
17 H 30 \\
18 H 10 \\
18 H 22 \\
18 H 58 \\
21 H 0 \\
O H O \\
O H 30 \\
O H 30 \\
0 H 44 \\
1 H 48 \\
2 H 34 \\
3 H 18 \\
4 H 4 \\
4 H 48 \\
5 H 22 \\
5 H 50 \\
6 H 40 \\
10 H 15 \\
11 H 42 \\
12 H 22 \\
13 H 30 \\
14 H 0 \\
14 H 12 \\
17 H 20 \\
18 H 8 \\
18 H 28 \\
19 H 0 \\
19 H 56\end{array}$ & 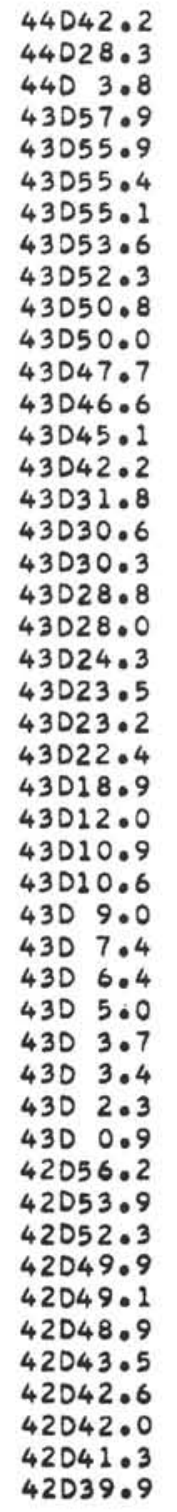 & 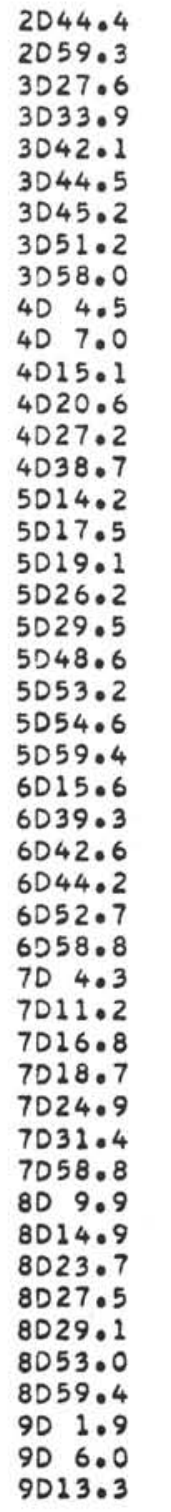 & 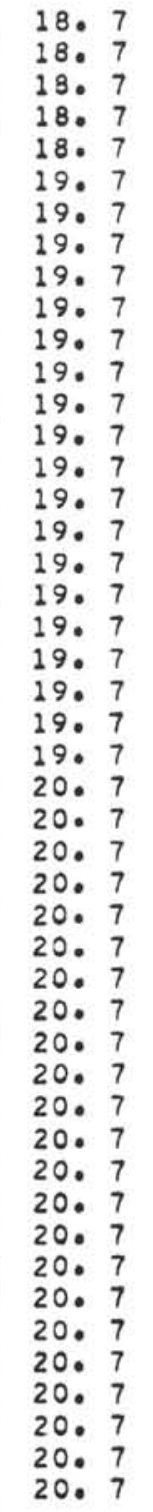 & 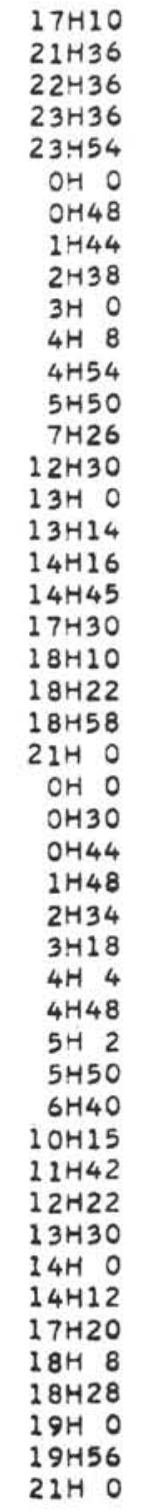 & $\begin{array}{l}44028 \cdot 3 \\
4403 \cdot 8 \\
43057 \cdot 9 \\
43055 \cdot 9 \\
43055 \cdot 4 \\
43055 \cdot 1 \\
43053 \cdot 6 \\
43052 \cdot 3 \\
43050 \cdot 8 \\
43050 \cdot 0 \\
43047 \cdot 7 \\
43046 \cdot 6 \\
43045 \cdot 1 \\
43042 \cdot 2 \\
43031 \cdot 8 \\
43030 \cdot 6 \\
43030 \cdot 3 \\
43028 \cdot 8 \\
43028 \cdot 0 \\
43024 \cdot 3 \\
43023 \cdot 5 \\
43023 \cdot 2 \\
43022 \cdot 4 \\
43018 \cdot 9 \\
43012 \cdot 0 \\
43010 \cdot 9 \\
43010 \cdot 6 \\
4309 \cdot 0 \\
4307 \cdot 4 \\
4306.4 \\
4305.0 \\
4303 \cdot 7 \\
430304 \\
4302 \cdot 3 \\
4300.9 \\
42056 \cdot 2 \\
42053 \cdot 9 \\
42052 \cdot 3 \\
42049 \cdot 9 \\
42049 \cdot 1 \\
42048 \cdot 9 \\
42043 \cdot 5 \\
42042 \cdot 6 \\
42042 \cdot 0 \\
42041 \cdot 3 \\
42039 \cdot 9 \\
42037 \cdot 9\end{array}$ & 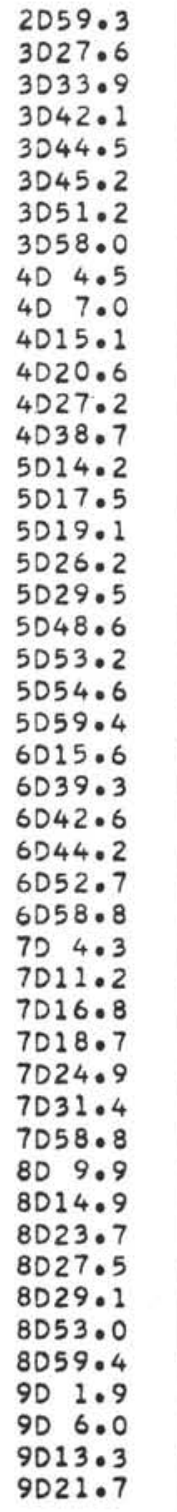 & 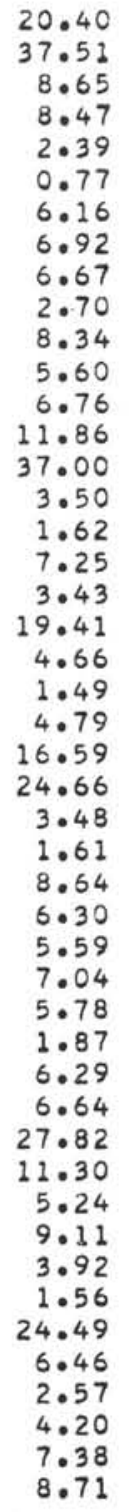 & 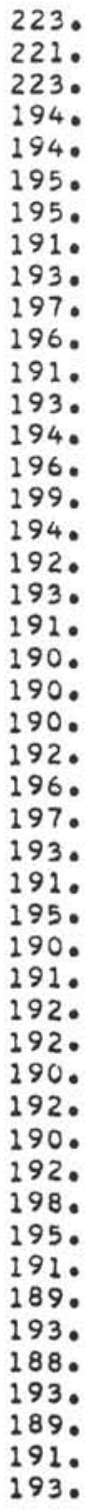 & $\begin{array}{l}8.562 \\
8.461 \\
8.651 \\
8.476 \\
7.979 \\
7.714 \\
7.710 \\
7.418 \\
7.413 \\
7.372 \\
7.362 \\
7.317 \\
7.253 \\
7.414 \\
7.302 \\
7.018 \\
6.943 \\
7.023 \\
7.100 \\
7.061 \\
7.003 \\
7.498 \\
7.995 \\
8.160 \\
8.223 \\
6.973 \\
6.912 \\
8.108 \\
8.225 \\
7.622 \\
9.182 \\
7.893 \\
8.055 \\
7.870 \\
7.978 \\
7.766 \\
7.797 \\
7.871 \\
8.046 \\
7.847 \\
7.839 \\
7.818 \\
8.077 \\
7.710 \\
7.889 \\
7.911 \\
8.167\end{array}$ & $\begin{array}{l}20.40 \\
57.92 \\
66.57 \\
75.04 \\
77.44 \\
78.21 \\
84.38 \\
91.30 \\
97.97 \\
100.68 \\
109.02 \\
114.63 \\
121.40 \\
133.26 \\
170.26 \\
173.77 \\
175.39 \\
182.65 \\
186.08 \\
205.50 \\
210.17 \\
211.67 \\
216.47 \\
233.06 \\
257.73 \\
261.22 \\
262.83 \\
271.48 \\
277.79 \\
283.38 \\
290.42 \\
296.21 \\
298.09 \\
304.38 \\
311.03 \\
338.86 \\
350.17 \\
355.41 \\
364.53 \\
358.45 \\
370.02 \\
394.52 \\
400.98 \\
403.55 \\
407.76 \\
415.14 \\
423.86\end{array}$ \\
\hline
\end{tabular}




\begin{tabular}{|c|c|c|c|c|c|c|c|c|c|c|c|}
\hline $\begin{array}{c}\text { DATE } \\
(\text { day/mo) }\end{array}$ & $\underset{(\mathrm{hr} / \mathrm{min})}{\mathrm{GMT}}$ & $\begin{array}{l}\text { LONGITUDE } \\
\text { (deg/min) }\end{array}$ & $\begin{array}{l}\text { LATITUDE } \\
\text { (deg/min) }\end{array}$ & $\begin{array}{c}\text { DATE } \\
\text { (day/mo) }\end{array}$ & $\begin{array}{c}\text { GMT } \\
(\mathrm{hr} / \mathrm{min})\end{array}$ & $\begin{array}{l}\text { LONGITUDE } \\
\text { (deg/min) }\end{array}$ & $\begin{array}{l}\text { LATITUDE } \\
\text { (deg/min) }\end{array}$ & $\begin{array}{c}\text { DIST } \\
(\mathrm{nmi})\end{array}$ & $\begin{array}{l}\text { CSE } \\
\left({ }^{\circ}\right)\end{array}$ & $\begin{array}{l}\text { SPEED } \\
(k t)\end{array}$ & $\begin{array}{l}\text { DISTT } \\
(\mathrm{nmi})\end{array}$ \\
\hline & & & & & & & & & & & \\
\hline $\begin{array}{l}20 \cdot 7 \\
20.7\end{array}$ & $\begin{array}{l}21 \mathrm{HO} \\
21 \mathrm{H}_{42}\end{array}$ & $\begin{array}{l}42037.9 \\
42036.2\end{array}$ & $\begin{array}{l}9021.7 \\
9027.3\end{array}$ & $\begin{array}{l}20.7 \\
20.7\end{array}$ & $\begin{array}{l}* 21 \mathrm{H} 42 \\
* \quad 23 \mathrm{H} 14\end{array}$ & $\begin{array}{l}42036 \cdot 2 \\
42033.4\end{array}$ & $\begin{array}{l}9027.3 \\
9039.6\end{array}$ & $\begin{array}{r}5.77 \\
12.61\end{array}$ & $\begin{array}{l}197 . \\
193 .\end{array}$ & $\begin{array}{l}8.246 \\
8.223\end{array}$ & $\begin{array}{l}429.63 \\
442.24\end{array}$ \\
\hline 20.7 & $23 \mathrm{H} 24$ & 42033.4 & 9039.6 & $20: 7$ & * $23 \mathrm{H} 48$ & 42031.9 & 9044.6 & 5.21 & 197. & 9.206 & 447.46 \\
\hline 20.7 & $23 \mathrm{H} 48$ & 42031.9 & 9044.6 & 21.7 & * OH O & 42031.4 & 9046.1 & 1.67 & 197. & 8.356 & 449.13 \\
\hline 21.7 & $\mathrm{OHO}$ & 42031.4 & 9046.1 & 21.7 & * OH54 & 42029.2 & 9053.4 & 7.52 & 197. & 8.363 & 456.66 \\
\hline 21.7 & OHS 4 & 42029.2 & 9053.4 & 21.7 & * 1 H36 & 42027.2 & 9059.0 & 5.94 & 199. & 8.486 & 462.60 \\
\hline 21.7 & $1 \mathrm{H} 36$ & 42027.2 & 9059.0 & 21.7 & * $1 \mathrm{H} 45$ & 42026.7 & 1000.1 & 1.27 & 202 . & 8.518 & 463.87 \\
\hline 21.7 & $1 H 45$ & 42026.7 & $100 \quad 0.1$ & 21.7 & * $2 \mathrm{H} 44$ & 42025.1 & 1008.1 & 8.07 & 191. & 8.213 & 471.95 \\
\hline 21.7 & $2 \mathrm{H} 44$ & 42025.1 & 1008.1 & 21.7 & * $\quad 3410$ & 42024.6 & 10011.8 & 3.76 & 186. & 8.698 & 475.72. \\
\hline 21.7 & $3 \mathrm{H} 1 \mathrm{O}$ & 42024.6 & 10011.8 & 21.7 & * 3 H18 & 42024.6 & 10013.0 & 1.15 & $184^{\circ}$ & 8.672 & 476.88 \\
\hline 21.7 & $3 \mathrm{H} 18$ & 42024.6 & 10013.0 & 21.7 & * $4 H^{H} 8$ & 42023.8 & 10020.5 & 7.54 & 186. & 9.050 & 484.42 \\
\hline 21.7 & $4 \mathrm{H}^{\circ}$ & 42023.8 & 10020.5 & 21.7 & $4 \mathrm{H} 35$ & 42023.1 & 10024.2 & 3.84 & 189. & 8.540 & 488.25 \\
\hline 21.7 & $4 \mathrm{H} 35$ & $42 D 23.1$ & 10024.2 & 21.7 & * $5 \mathrm{HO}$ & 42022.7 & 10027.8 & 3.53 & 188. & 8.491 & 491.80 \\
\hline 21. 7 & $5 H_{0}$ & 42022.7 & 10027.8 & 21.7 & * 6H52 & 42021.5 & 10044.3 & 16.54 & 184. & 8.862 & 508.34 \\
\hline 21.7 & $6 \mathrm{H} 52$ & 42021.5 & 10044.3 & 21.7 & * $\quad 7 \mathrm{H} 23$ & 42020.3 & 10048.4 & 4.30 & 195. & 8.330 & 512.55 \\
\hline 21.7 & $7 \mathrm{H} 23$ & 42020.3 & 10048.4 & 21.7 & * $7 \mathrm{H} 36$ & 42020.0 & 10050.2 & 1.78 & 193. & 8.216 & 514.43 \\
\hline 21.7 & $7 H 36$ & 42020.0 & 10050.2 & 21.7 & * $8 \mathrm{H} 10$ & 42019.5 & 10055.1 & 4.96 & 185. & 8.754 & 519.39 \\
\hline 21.7 & $8 \mathrm{H} 10$ & 42019.5 & 10055.1 & 21.7 & * $8 \mathrm{H} 25$ & 42019.4 & 10057.3 & 2.16 & 182. & 8.679 & 521.56 \\
\hline 21.7 & $8 \mathrm{H} 25$ & 42019.4 & 10057.3 & 21.7 & * 11 H32 & 42018.7 & 11025.3 & 28.00 & 182. & 8.983 & 549.56 \\
\hline 21.7 & $11 H 32$ & 42018.7 & 11025.3 & 21.7 & * $12 \mathrm{H} 40$ & 42018.1 & 11035.7 & 10.47 & 183. & 9.239 & 560.03 \\
\hline 21.7 & $12 \mathrm{H} 4 \mathrm{O}^{\circ}$ & 42018.1 & 11035.7 & 21.7 & * $12 \mathrm{H} 46$ & 42018.0 & 11036.7 & 0.95 & 188. & 9.523 & 560.98 \\
\hline 21.7 & $12 \mathrm{H} 46$ & 42018.0 & 11036.7 & 21.7 & * $13 \mathrm{H} 14$ & 42018.4 & 11041.2 & 4.51 & 175. & 9.679 & 565.50 \\
\hline 21.7 & $13 \mathrm{H} 14$ & 42018.4 & 11041.2 & 21.7 & * 13 H3O & 42018.2 & 11043.5 & 2.36 & 183. & 8.869 & 567.86 \\
\hline 21.7 & $13 \mathrm{H} 3 \mathrm{O}$ & 42018.2 & 11043.5 & 21.7 & * $15 \mathrm{HS2}$ & 42015.2 & 1204.5 & 21.15 & 188. & 8.939 & 589.02 \\
\hline 21.7 & $15 \mathrm{H} 52$ & 42015.2 & 1204.5 & 21.7 & * $16 \mathrm{H} 32$ & 42014.4 & 12010.6 & 6.15 & 187. & 9.225 & 595.17 \\
\hline 21.7 & $16 H 32$ & 42014.4 & 12010.6 & 21.7 & * $17 \mathrm{H} 3$ & 42013.9 & 12015.1 & 4.58 & 185. & 8.877 & 599.76 \\
\hline 21.7 & $17 \mathrm{H}_{3}$ & 42013.9 & 12015.1 & 21.7 & * $17 \mathrm{H} 36$ & 42013.2 & 12020.0 & 4.89 & 189. & 8.897 & 604.65 \\
\hline 21.7 & 17436 & 42013.2 & 12020.0 & 21.7 & * $18 \mathrm{H} 18$ & $42 D 12.8$ & 12026.4 & 6.41 & 184. & 9.160 & 611.06 \\
\hline 21.7 & $18 \mathrm{H} 18$ & 42012.8 & 12026.4 & 21.7 & * $18 H 50$ & 42012.5 & 12031.2 & 4.89 & 183. & 9.168 & 615.95 \\
\hline 21.7 & 18 H5O & 42012.5 & 12031.2 & 21.7 & * $19 H_{2} 2$ & 42012.3 & 12033.1 & $\begin{array}{l}1.82 \\
1.82\end{array}$ & 186. & 9.149 & 617.78 \\
\hline 21.7 & $19 H_{2} 2$ & 42012.3 & 12033.1 & 21.7 & * $19 \mathrm{H}+30$ & 42011.8 & 12037.3 & 4.31 & 186. & 9.249 & 622.10 \\
\hline 21. 7 & $19 \mathrm{H}_{3} \mathrm{O}^{\circ}$ & 42011.8 & 12037.3 & 22.7 & * OH O & 4203.6 & 13017.9 & 41.35 & 191. & 9.193 & 663.47 \\
\hline 22.7 & $\mathrm{OHO}$ & 4203.6 & 13017.9 & 22.7 & * OH 6 & 4203.5 & 13018.9 & 0.93 & 191. & 9.354 & 664.40 \\
\hline 22. 7 & $\mathrm{OH} 6$ & 4203.5 & 13018.9 & 22.7 & * $0 \mathrm{H} 42$ & 4202.3 & 13024.1 & 5.33 & 193. & 8.885 & 669.74 \\
\hline 22.7 & $\mathrm{OH}_{42}$ & $420 \quad 2.3$ & 1302401 & 22.7 & * $1 \mathrm{H} 56$ & 4200.3 & 13034.6 & 10.68 & 191. & 8.660 & 680.42 \\
\hline 22. 7 & $2 H 56$ & 4200.3 & 13034.6 & 22. 7 & * $3 \mathrm{H}_{18}$ & 41057.3 & 13045.7 & 11.48 & 195. & 8.400 & 691.90 \\
\hline 22.7 & $3 \mathrm{H} 18$ & 41057.3 & 13045.7 & 22. 7 & * $4 \mathrm{H} 18$ & 41055.4 & 13054.1 & 8.60 & 192. & 8.602 & 700.50 \\
\hline 22.7 & $4 H 18$ & 41055.4 & 13054.1 & 22.7 & * $6 \mathrm{H} 0$ & 41051.9 & 1407.1 & 13.44 & 195. & 7.906 & 713.94 \\
\hline 22. 7 & $6 \mathrm{HO}$ & 41051.9 & 1407.1 & 22. 7 . & * $6 \mathrm{H} 45$ & 41050.1 & 14012.9 & 6.08 & 196. & 8.109 & 720.02 \\
\hline 22. 7 & $6 \mathrm{H} 45$ & 41050.1 & 14012.9 & 22. 7 & * $8 \mathrm{H} 34$ & 41047.9 & $14 D 27.5$ & 14.72 & 189. & 8.103 & 734.74 \\
\hline 22.7 & $8 \mathrm{H} 34$ & 41047.9 & 14027.5 & 22. 7 & * $11 \mathrm{H56}$ & 41043.2 & 14054.5 & 27.38 & 190. & 8.134 & 762.13 \\
\hline 22. 7 & 11436 & 41043.2 & 14054.5 & 22. 7 & * $12 \mathrm{H} 26$ & 41042.2 & 14059.3 & 4.89 & 191. & 9.794 & 757.03 \\
\hline 22. 7 & $12 \mathrm{H} 26$ & 41042.2 & 14059.3 & 22. 7 & * 13 H38 & 41040.1 & 1509.4 & 10.30 & 191. & 8.586 & 777.33 \\
\hline 22.7 & $13 \mathrm{H} 38$ & 41040.1 & 1509.4 & 22.7 & * $14 H 26$ & 41038.6 & 15015.9 & 6.70 & 192. & 8.387 & 784.04 \\
\hline 22.7 & $14 \mathrm{H} 26$ & 41038.6 & 1501509 & 22.7 & * $14 \mathrm{H} 37$ & 41038.3 & 15017.4 & 1.53 & 191. & 8.379 & 785.58 \\
\hline 22. 7 & $14 \mathrm{H} 37$ & 41038.3 & 15017.4 & 22. 7 & * $15 \mathrm{H}^{7} 7$ & 41036.7 & 15021.4 & 4.23 & 202 . & 8.479 & 789.82 \\
\hline 22. 7 & $15 \mathrm{H}^{7}$ & 41036.7 & 15021.4 & 22. 7 & $15 \mathrm{H} 42$ & 41035.8 & 15026.2 & 4.88 & 191. & 8.369 & 794.70 \\
\hline
\end{tabular}


TABLE 7 - Continued

\begin{tabular}{|c|c|c|c|c|c|c|c|c|c|c|c|}
\hline $\begin{array}{c}\text { DATE } \\
\text { (day/mo) }\end{array}$ & $\underset{(\mathrm{hr} / \mathrm{m} \cdot \mathrm{in})}{\mathrm{GMT}}$ & $\begin{array}{l}\text { LONGITUDE } \\
\text { (deg/min) }\end{array}$ & $\begin{array}{l}\text { LATITUDE } \\
\text { (deg/min) }\end{array}$ & $\begin{array}{c}\text { DATE } \\
\text { (day/mo) }\end{array}$ & $\begin{array}{c}\text { GMT } \\
(\mathrm{hr} / \mathrm{min})\end{array}$ & $\begin{array}{l}\text { LONGITUDE } \\
\text { (deg/min) }\end{array}$ & $\begin{array}{l}\text { LATITUDE } \\
\text { (deg/min) }\end{array}$ & $\begin{array}{c}\text { DIST } \\
(\mathrm{nmi})\end{array}$ & $\begin{array}{l}\text { CSE } \\
\left({ }^{\circ}\right)\end{array}$ & $\begin{array}{l}\text { SPEED } \\
(k t)\end{array}$ & $\begin{array}{l}\text { DISTT } \\
(n m i)\end{array}$ \\
\hline $\begin{array}{ll}220 & 7 \\
220 & 7 \\
220 & 7 \\
220 & 7 \\
220 & 7 \\
220 & 7\end{array}$ & $\begin{array}{l}15 \mathrm{H} 42 \\
16 \mathrm{H} 23 \\
16 \mathrm{H} 44 \\
17 \mathrm{H} 28 \\
18 \mathrm{H} 14 \\
18 \mathrm{H} 36\end{array}$ & $\begin{array}{l}41035.8 \\
41035.9 \\
41036.2 \\
41035.5 \\
41034.6 \\
41034.2\end{array}$ & $\begin{array}{l}15026.2 \\
15031.4 \\
15034.2 \\
15040.0 \\
15046.3 \\
15049.3\end{array}$ & $\begin{array}{l}22 \cdot 7 \\
22 \cdot 7 \\
2207 \\
22 \cdot 7 \\
22 \cdot 7 \\
22 \cdot 7\end{array}$ & $\begin{array}{l}16 \mathrm{H} 23 \\
16 \mathrm{H} 44 \\
17 \mathrm{H} 28 \\
18 \mathrm{H} 14 \\
18 \mathrm{H} 36 \\
19 \mathrm{H} 20\end{array}$ & $\begin{array}{l}41035.9 \\
41036.2 \\
41035.5 \\
41034.6 \\
41034.2 \\
41032.9\end{array}$ & $\begin{array}{l}15031.4 \\
15034 \cdot 2 \\
15040.0 \\
15046.3 \\
15049.3 \\
15055.7\end{array}$ & $\begin{array}{l}5.27 \\
2.73 \\
5.83 \\
6.36 \\
3.02 \\
6.55\end{array}$ & $\begin{array}{l}178^{\circ} \\
175^{\circ} \\
1870^{\circ} \\
188^{\circ} \\
187^{\circ} \\
191^{\circ}\end{array}$ & $\begin{array}{l}7.722 \\
7.811 \\
7.963 \\
8.295 \\
8.249 \\
8.931\end{array}$ & $\begin{array}{l}799.98 \\
802.71 \\
808.55 \\
814.91 \\
817.93 \\
824.48\end{array}$ \\
\hline
\end{tabular}


TABLE 8

Glomar Challenger, DSDP Leg 25, Navigation Data

\begin{tabular}{|c|c|c|c|c|c|c|c|c|c|c|c|}
\hline $\begin{array}{c}\text { DATE } \\
\text { (day/mo) }\end{array}$ & $\begin{array}{c}\text { GMT } \\
(\mathrm{hr} / \mathrm{min})\end{array}$ & $\begin{array}{l}\text { LONGITUDE } \\
\text { (deg/min) }\end{array}$ & $\begin{array}{l}\text { LATITUDE } \\
\text { (deg/min) }\end{array}$ & $\begin{array}{c}\text { DATE } \\
(\text { day/mo) }\end{array}$ & $\begin{array}{c}\text { GMT } \\
(\mathrm{hr} / \mathrm{min})\end{array}$ & $\begin{array}{l}\text { LONGITUDE } \\
\text { (deg/min) }\end{array}$ & $\begin{array}{l}\text { LATITUDE } \\
\text { (deg/min) }\end{array}$ & $\begin{array}{l}\text { DIST } \\
(n m i)\end{array}$ & $\begin{array}{l}\text { CSE } \\
\left({ }^{\circ}\right)\end{array}$ & $\begin{array}{l}\text { SPEED } \\
(k t)\end{array}$ & $\begin{array}{l}\text { DISTT } \\
\text { (nmi) }\end{array}$ \\
\hline 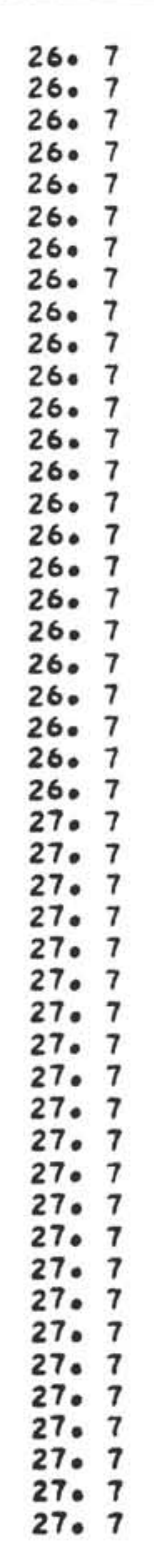 & $\begin{array}{l}3 H 10 \\
3 H 22 \\
3 H 30 \\
4 H 36 \\
5 H 10 \\
5 H 50 \\
6 H 24 \\
7 H 06 \\
8 H 00 \\
11 H 30 \\
12 H 12 \\
13 H 21 \\
13 H 25 \\
13 H 43 \\
13 H 56 \\
15 H 10 \\
16 H 0 \\
16 H 30 \\
17 H 48 \\
18 H 30 \\
19 H 0 \\
20 H 18 \\
20 H 50 \\
22 H 0 \\
0 H 0 \\
1 H 0 \\
1 H 20 \\
1 H 22 \\
2 H 15 \\
3 H 48 \\
4 H 16 \\
4 H 43 \\
5 H 34 \\
6 H 44 \\
6 H 54 \\
8 H 22 \\
8 H 47 \\
11 H 22 \\
12 H 8 \\
13 H 06 \\
13 H 21 \\
16 H 22 \\
16 H 58 \\
17 H 00 \\
17 H 50 \\
19 H 10 \\
19 H 26 \\
21 H 18\end{array}$ & $\begin{array}{l}41049 \cdot 2 \\
41049 \cdot 3 \\
41049 \cdot 4 \\
41049 \cdot 1 \\
41048 \cdot 3 \\
41047 \cdot 9 \\
41048 \cdot 1 \\
41048 \cdot 8 \\
41049 \cdot 6 \\
41049 \cdot 5 \\
41049 \cdot 0 \\
41048 \cdot 3 \\
41048 \cdot 1 \\
41047 \cdot 0 \\
41046 \cdot 9 \\
41047 \cdot 0 \\
41047 \cdot 0 \\
41047 \cdot 1 \\
41047 \cdot 1 \\
41047 \cdot 3 \\
41047 \cdot 4 \\
41047 \cdot 2 \\
41047 \cdot 0 \\
41046 \cdot 0 \\
41041 \cdot 5 \\
41039 \cdot 3 \\
41039 \cdot 0 \\
41037 \cdot 8 \\
41036 \cdot 3 \\
41036 \cdot 2 \\
41035 \cdot 6 \\
41035 \cdot 2 \\
41034 \cdot 5 \\
41034 \cdot 2 \\
41034 \cdot 6 \\
41034 \cdot 8 \\
41034 \cdot 9 \\
41034 \cdot 6 \\
41033 \cdot 6 \\
41033 \cdot 1 \\
41029 \cdot 2 \\
41027 \cdot 9 \\
41027 \cdot 8 \\
41028 \cdot 1 \\
41028 \cdot 5 \\
41028 \cdot 7 \\
41029 \cdot 7\end{array}$ & 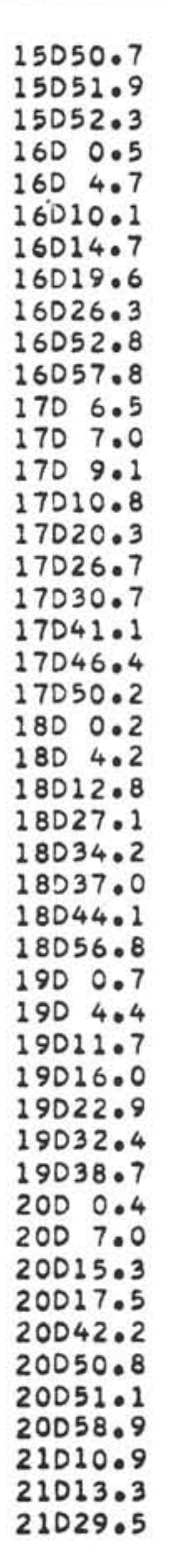 & 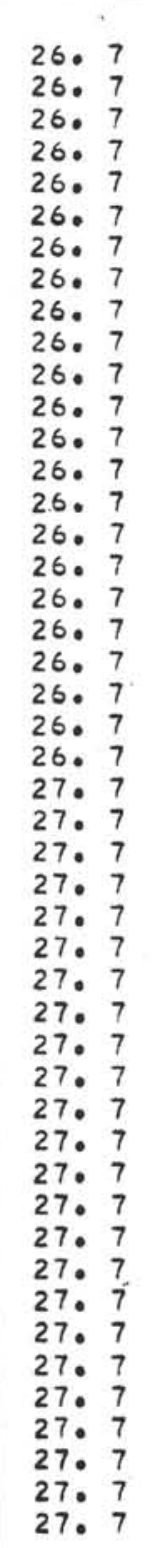 & $\begin{array}{r}3 H 22 \\
3 H 30 \\
4 H 36 \\
5 H 10 \\
5 H 50 \\
6 H 24 \\
7 H 06 \\
8 H 00 \\
11 H 30 \\
12 H 12 \\
13 H 21 \\
13 H 25 \\
13 H 43 \\
13 H 56 \\
15 H 10 \\
16 H 0 \\
16 H 30 \\
17 H 48 \\
18 H 30 \\
19 H 0 \\
20 H 18 \\
20 H 50 \\
22 H 0 \\
0 H 0 \\
1 H 0 \\
1 H 22 \\
1 H H 15 \\
2 H H 4 \\
3 H 48 \\
4 H 16 \\
4 H 43 \\
5 H 34 \\
6 H \quad 4 \\
6 H 54 \\
8 H 2 \\
8 H 47 \\
11 H 22 \\
12 H 0 \\
13 H 06 \\
13 H 21 \\
16 H 2 \\
16 H 58 \\
17 H 00 \\
17 H 50 \\
19 H 10 \\
19 H 26 \\
21 H 18 \\
21 H 48\end{array}$ & $\begin{array}{l}41049 \cdot 3 \\
41049 \cdot 4 \\
41049 \cdot 1 \\
41048 \cdot 3 \\
41047 \cdot 9 \\
41048 \cdot 1 \\
41048 \cdot 8 \\
41049 \cdot 6 \\
41049 \cdot 5 \\
41049 \cdot 0 \\
41048 \cdot 3 \\
41048 \cdot 1 \\
41047 \cdot 0 \\
41046 \cdot 9 \\
41047 \cdot 0 \\
41047 \cdot 0 \\
41047 \cdot 1 \\
41047 \cdot 1 \\
41047 \cdot 3 \\
41047 \cdot 4 \\
41047 \cdot 2 \\
41047 \cdot 0 \\
41046 \cdot 0 \\
4104.1 \cdot 5 \\
41039 \cdot 3 \\
41039 \cdot 0 \\
41037 \cdot 8 \\
41036 \cdot 3 \\
41036 \cdot 2 \\
41035 \cdot 6 \\
41035 \cdot 2 \\
41034 \cdot 5 \\
41034 \cdot 2 \\
41034 \cdot 6 \\
41034 \cdot 8 \\
41034 \cdot 9 \\
41034 \cdot 6 \\
41033 \cdot 6 \\
41033 \cdot 1 \\
41029 \cdot 2 \\
41027 \cdot 9 \\
41027 \cdot 8 \\
41028 \cdot 1 \\
41028 \cdot 5 \\
41028 \cdot 7 \\
41029 \cdot 7 \\
41029 \cdot 7\end{array}$ & 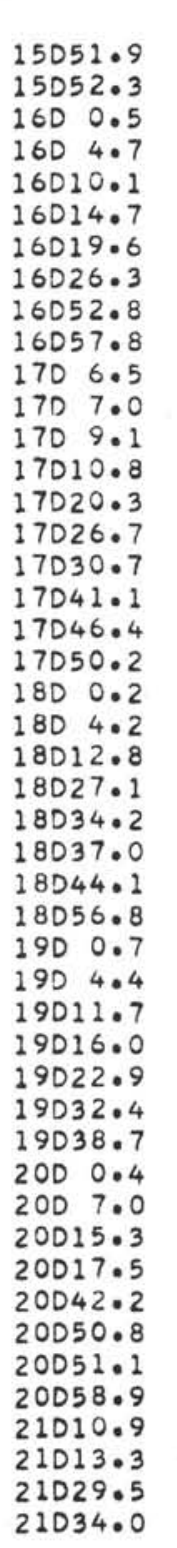 & $\begin{array}{r}1.20 \\
0.49 \\
8.13 \\
4.27 \\
5.44 \\
4.56 \\
4.94 \\
6.76 \\
26.50 \\
5.02 \\
8.76 \\
0.51 \\
2.38 \\
1.65 \\
9.50 \\
6.40 \\
4.000 \\
10.39 \\
5.30 \\
3.84 \\
9.96 \\
4.00 \\
8.72 \\
14.89 \\
7.44 \\
2.75 \\
7.24 \\
12.72 \\
3.90 \\
3.82 \\
7.22 \\
4.35 \\
6.93 \\
9.47 \\
6.31 \\
21.69 \\
6.62 \\
8.34 \\
2.32 \\
24.89 \\
8.68 \\
0.31 \\
7.79 \\
12.00 \\
2.40 \\
16.22 \\
4.56\end{array}$ & 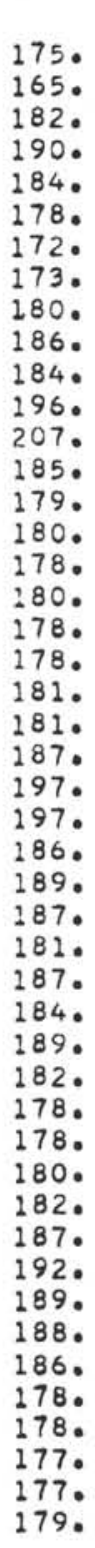 & $\begin{array}{l}6.019 \\
3.679 \\
7.392 \\
7.536 \\
8.170 \\
8.060 \\
7.066 \\
7.512 \\
7.572 \\
7.174 \\
7.618 \\
7.758 \\
7.940 \\
7.637 \\
7.703 \\
7.680 \\
8.009 \\
7.998 \\
7.576 \\
7.689 \\
7.662 \\
7.512 \\
7.477 \\
7.445 \\
7.445 \\
7.506 \\
8.202 \\
8.209 \\
8.359 \\
8.489 \\
8.499 \\
8.702 \\
8.323 \\
8.362 \\
8.416 \\
8.397 \\
8.642 \\
8.628 \\
9.307 \\
9.277 \\
9.306 \\
9.360 \\
9.350 \\
9.007 \\
9.007 \\
8.697 \\
9.133\end{array}$ & $\begin{array}{r}1.20 \\
1.59 \\
9.82 \\
14.09 \\
19.54 \\
24.11 \\
29.05 \\
35.81 \\
62.32 \\
67.34 \\
76.10 \\
76.62 \\
79.00 \\
80.65 \\
90.15 \\
96.55 \\
100.56 \\
110.96 \\
116.26 \\
120.11 \\
130.07 \\
134.07 \\
142.80 \\
157.69 \\
165.13 \\
157.89 \\
175.13 \\
187.86 \\
191.75 \\
195.58 \\
202.80 \\
207.15 \\
214.09 \\
223.57 \\
229.88 \\
251.59 \\
258.20 \\
266.54 \\
268.87 \\
293.76 \\
302.45 \\
302.76 \\
310.55 \\
322.56 \\
324.96 \\
341.19 \\
345.76\end{array}$ \\
\hline
\end{tabular}


TABLE 8 - Continued

\begin{tabular}{|c|c|c|c|c|c|c|c|c|c|c|c|}
\hline $\begin{array}{c}\text { DATE } \\
\text { (day/mo) }\end{array}$ & $\underset{(\mathrm{hr} / \mathrm{min})}{\text { GMT }}$ & $\begin{array}{l}\text { LONGITUDE } \\
\text { (deg/min) }\end{array}$ & $\begin{array}{l}\text { LATITUDE } \\
\text { (deg/min) }\end{array}$ & $\begin{array}{c}\text { DATE } \\
\text { (day/mo) }\end{array}$ & $\begin{array}{c}\text { GMT } \\
(\mathrm{hr} / \mathrm{min})\end{array}$ & $\begin{array}{l}\text { LONGITUDE } \\
(\mathrm{deg} / \mathrm{min})\end{array}$ & $\begin{array}{l}\text { LATITUDE } \\
\text { (deg/min })\end{array}$ & $\begin{array}{r}\text { DIST } \\
(\mathrm{nmi})\end{array}$ & $\begin{array}{l}\text { CSE } \\
\left({ }^{\circ}\right)\end{array}$ & $\begin{array}{l}\text { SPEED } \\
(k t)\end{array}$ & $\begin{array}{l}\text { DISTT } \\
(n m i)\end{array}$ \\
\hline $\begin{array}{l}27: 7 \\
27: 7 \\
2807 \\
2807 \\
2807 \\
2807 \\
28: 7 \\
2807 \\
2807 \\
2807 \\
2807 \\
28: 7\end{array}$ & $\begin{array}{r}21 H_{4} 8 \\
22 \mathrm{H} 42 \\
\mathrm{OH} 0 \\
\mathrm{OH} 24 \\
1 \mathrm{H} 10 \\
2 \mathrm{H} 24 \\
2 \mathrm{H} 41 \\
3 \mathrm{H} 24 \\
4 \mathrm{H} 46 \\
5 \mathrm{H} 12 \\
5 \mathrm{H} 43 \\
7 \mathrm{H} 16\end{array}$ & $\begin{array}{l}41029.7 \\
41029.4 \\
41028.2 \\
41027.9 \\
41027.8 \\
41029.0 \\
41029.3 \\
41029.5 \\
41027.6 \\
41029.0 \\
41029.0 \\
41031.2\end{array}$ & $\begin{array}{l}21034.0 \\
21042.3 \\
21053.0 \\
21056.3 \\
2202.7 \\
22011.6 \\
22015.3 \\
22021.4 \\
22032.5 \\
22036.2 \\
22040.5 \\
22053.4\end{array}$ & 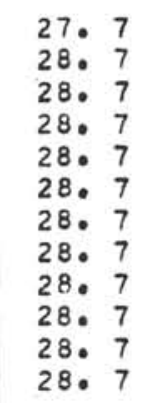 & $\begin{array}{c}22 \mathrm{H} 42 \\
\mathrm{OH} 0 \\
\mathrm{OH} 24 \\
1 \mathrm{H} 10 \\
2 \mathrm{H} 14 \\
2 \mathrm{H} 41 \\
3 \mathrm{H} 24 \\
4 \mathrm{H} 46 \\
5 \mathrm{H} 12 \\
5 \mathrm{H} 43 \\
7 \mathrm{H} 16 \\
7 \mathrm{H} 48\end{array}$ & $\begin{array}{l}41029 \cdot 4 \\
41028 \cdot 2 \\
41027 \cdot 9 \\
41027 \cdot 8 \\
41029 \cdot 0 \\
41029 \cdot 3 \\
41029 \cdot 5 \\
41027 \cdot 6 \\
41029 \cdot 0 \\
41029 \cdot 0 \\
41031 \cdot 2 \\
41031 \cdot 7\end{array}$ & $\begin{array}{l}21042.3 \\
21053.0 \\
21056.3 \\
2202.7 \\
22011.6 \\
22015.3 \\
22021.4 \\
22032.5 \\
22036.2 \\
22040.5 \\
22053.4 \\
22057.9\end{array}$ & $\begin{array}{r}8.23 \\
10.75 \\
3.31 \\
6.42 \\
8.93 \\
3.78 \\
6.03 \\
11.23 \\
3.92 \\
4.35 \\
13.00 \\
4.57\end{array}$ & $\begin{array}{l}182^{\circ} \\
186^{\circ} \\
186^{\circ} \\
180^{\circ} \\
173^{\circ} \\
175^{\circ} \\
179^{\circ} \\
189^{\circ} \\
161^{\circ} \\
1790^{\circ} \\
171^{\circ} \\
173^{\circ}\end{array}$ & $\begin{array}{l}9.155 \\
8.275 \\
8.279 \\
8.375 \\
8.380 \\
8.405 \\
8.417 \\
8.224 \\
9.050 \\
8.419 \\
8.387 \\
8.570\end{array}$ & $\begin{array}{l}354.00 \\
364.75 \\
368.07 \\
374.49 \\
383.43 \\
387.21 \\
393.24 \\
404.48 \\
408.40 \\
412.76 \\
425.76 \\
430.33\end{array}$ \\
\hline
\end{tabular}


TABLE 9

Glomar Challenger, DSDP Leg 25, Navigation Data

\begin{tabular}{|c|c|c|c|c|c|c|c|c|c|c|c|}
\hline $\begin{array}{c}\text { DATE } \\
\text { (day/mo) }\end{array}$ & $\underset{(\mathrm{hr} / \mathrm{min}) .}{\text { GMT }}$ & $\begin{array}{l}\text { LONGITUDE } \\
\text { (deg/min) }\end{array}$ & $\begin{array}{l}\text { LATITUDE } \\
\text { (deg/min) }\end{array}$ & $\begin{array}{c}\text { DATE } \\
\text { (day/mo) }\end{array}$ & $\begin{array}{c}\text { GMT } \\
(\mathrm{hr} / \mathrm{min})\end{array}$ & $\begin{array}{l}\text { LONGITUDE } \\
\text { (deg/min) }\end{array}$ & $\begin{array}{l}\text { LATITUDE } \\
(\mathrm{deg} / \mathrm{min})\end{array}$ & $\begin{array}{c}\text { DIST } \\
(\mathrm{nmi})\end{array}$ & $\begin{array}{l}\text { CSE } \\
\left({ }^{\circ}\right)\end{array}$ & $\begin{array}{l}\text { SPEED } \\
(k t)\end{array}$ & $\begin{array}{l}\text { DISTT } \\
(n m i)\end{array}$ \\
\hline 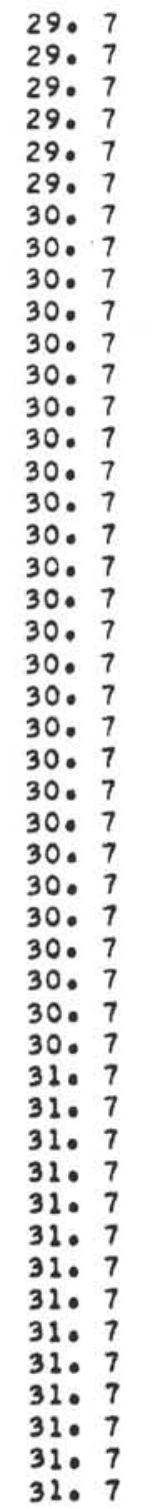 & 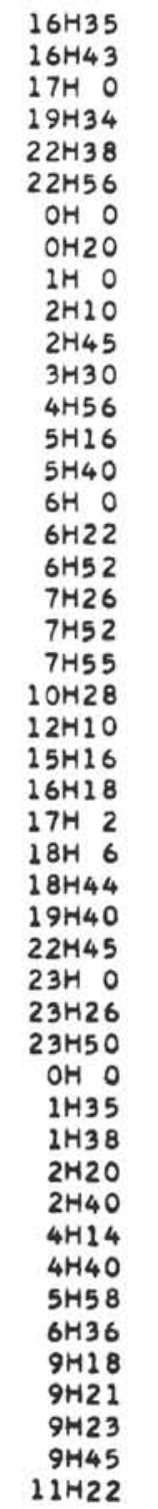 & 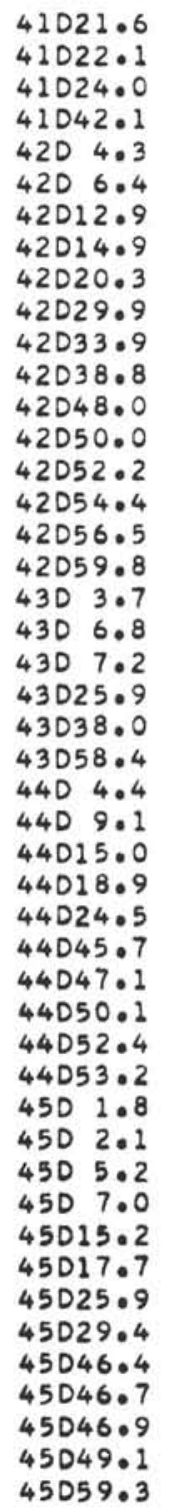 & $\begin{array}{l}22055 \cdot 6 \\
22056 \cdot 1 \\
22057 \cdot 8 \\
23014 \cdot 3 \\
23033 \cdot 2 \\
23034 \cdot 9 \\
23041 \cdot 7 \\
23043 \cdot 9 \\
23048 \cdot 0 \\
23055 \cdot 1 \\
23058 \cdot 4 \\
2403 \cdot 1 \\
24013 \cdot 1 \\
24015 \cdot 6 \\
24018 \cdot 5 \\
24021 \cdot 1 \\
24023 \cdot 6 \\
24027 \cdot 4 \\
24031 \cdot 6 \\
24034 \cdot 6 \\
24034 \cdot 9 \\
24052 \cdot 9 \\
2504 \cdot 0 \\
25023 \cdot 1 \\
25029 \cdot 7 \\
25034 \cdot 1 \\
25041 \cdot 2 \\
25045 \cdot 4 \\
25050 \cdot 9 \\
2607.1 \\
2608.8 \\
26011 \cdot 1 \\
26013 \cdot 3 \\
26014 \cdot 1 \\
26022 \cdot 3 \\
26022 \cdot 6 \\
26026 \cdot 3 \\
26027 \cdot 8 \\
26034 \cdot 3 \\
26036 \cdot 4 \\
26042 \cdot 3 \\
26044 \cdot 8 \\
26058 \cdot 1 \\
26058 \cdot 1 \\
26058 \cdot 0 \\
26057 \cdot 2 \\
2705 \cdot 2\end{array}$ & 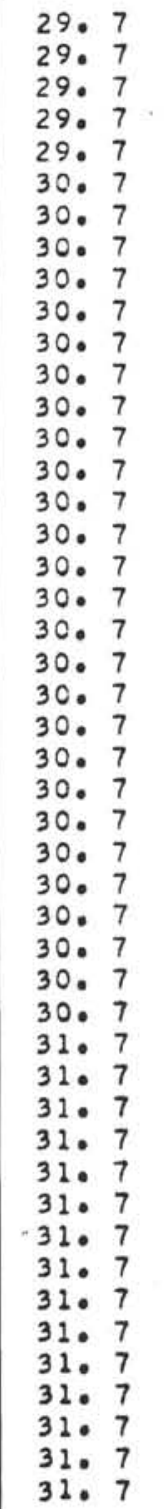 & $\begin{array}{r}16 H 43 \\
17 H 0 \\
19 H 34 \\
22 H 38 \\
22 H 56 \\
0 H 0 \\
0 H 20 \\
1 H 00 \\
2 H 10 \\
2 H 45 \\
3 H 30 \\
4 H 56 \\
5 H 16 \\
5 H 40 \\
6 H 0 \\
6 H 22 \\
6 H 52 \\
7 H 26 \\
7 H 52 \\
7 H 55 \\
10 H 28 \\
12 H 10 \\
15 H 16 \\
16 H 18 \\
17 H 2 \\
18 H 06 \\
18 H 44 \\
19 H 40 \\
22 H 45 \\
23 H 0 \\
23 H 26 \\
23 H 50 \\
0 H 0 \\
1 H 35 \\
1 H 338 \\
2 H 20 \\
2 H 40 \\
4 H 14 \\
4 H 40 \\
5 H 58 \\
6 H 36 \\
9 H 18 \\
9 H 21 \\
9 H 23 \\
9 H 45 \\
11 H 22 \\
13 H 0\end{array}$ & 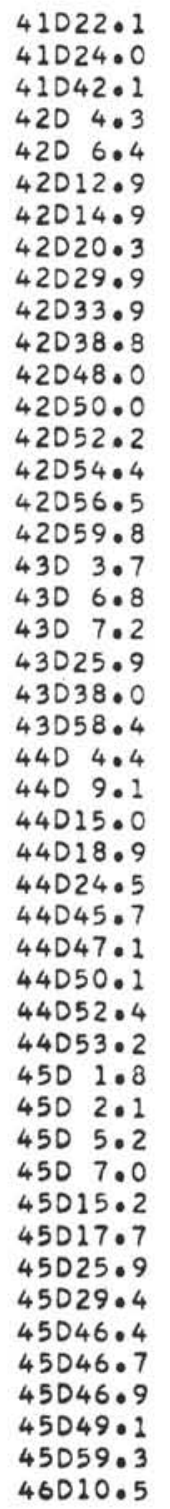 & $\begin{array}{l}22056 \cdot 1 \\
22057 \cdot 8 \\
23014 \cdot 3 \\
23033 \cdot 2 \\
23034 \cdot 9 \\
23041 \cdot 7 \\
23043 \cdot 9 \\
23048 \cdot 0 \\
23055 \cdot 1 \\
23058 \cdot 4 \\
2403 \cdot 1 \\
24013 \cdot 1 \\
24 D 15 \cdot 6 \\
24 D 18 \cdot 5 \\
24021 \cdot 1 \\
24023 \cdot 6 \\
24027 \cdot 4 \\
24031 \cdot 6 \\
24034 \cdot 6 \\
24034 \cdot 9 \\
24052 \cdot 9 \\
2504 \cdot 0 \\
25023 \cdot 1 \\
25029 \cdot 7 \\
25034 \cdot 1 \\
25041 \cdot 2 \\
25045 \cdot 4 \\
25050 \cdot 9 \\
2607 \cdot 1 \\
2608 \cdot 8 \\
26011 \cdot 1 \\
26013 \cdot 3 \\
26014 \cdot 1 \\
26022 \cdot 3 \\
26022 \cdot 6 \\
26026 \cdot 3 \\
26027 \cdot 8 \\
26034 \cdot 3 \\
26036 \cdot 4 \\
26042 \cdot 3 \\
26044 \cdot 8 \\
26058 \cdot 1 \\
26 D 58 \cdot 1 \\
26058 \cdot 0 \\
26057 \cdot 2 \\
2705 \cdot 2 \\
27013 \cdot 3\end{array}$ & $\begin{array}{r}0.70 \\
2.49 \\
23.39 \\
27.87 \\
2.57 \\
9.08 \\
2.84 \\
6.46 \\
11.31 \\
5.01 \\
6.43 \\
13.08 \\
3.10 \\
3.53 \\
3.32 \\
3.17 \\
4.83 \\
5.47 \\
4.20 \\
0.46 \\
24.74 \\
15.64 \\
26.63 \\
8.55 \\
6.12 \\
8.88 \\
5.48 \\
7.59 \\
25.06 \\
2.03 \\
3.52 \\
3.02 \\
1.18 \\
11.25 \\
0.32 \\
4.067 \\
2.15 \\
9.83 \\
3.16 \\
9.35 \\
4.01 \\
20.28 \\
0.29 \\
0.19 \\
2.14 \\
12.09 \\
12.91\end{array}$ & 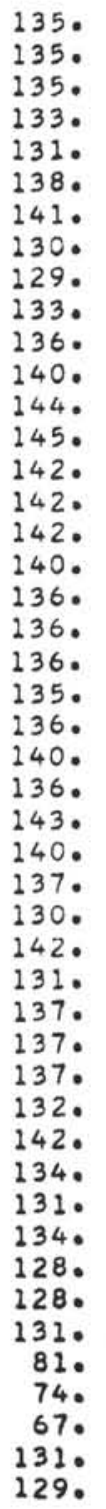 & $\begin{array}{l}5.313 \\
8.812 \\
9.112 \\
9.090 \\
8.587 \\
8.520 \\
8.527 \\
9.690 \\
9.696 \\
8.591 \\
8.577 \\
9.130 \\
9.301 \\
8.830 \\
9.967 \\
8.670 \\
9.668 \\
9.659 \\
9.703 \\
9.203 \\
9.704 \\
9.204 \\
8.590 \\
8.282 \\
8.355 \\
8.333 \\
8.665 \\
8.142 \\
8.129 \\
8.159 \\
8.131 \\
7.560 \\
7.111 \\
7.110 \\
6.446 \\
6.683 \\
6.471 \\
6.275 \\
7.299 \\
7.196 \\
6.341 \\
7.513 \\
5.809 \\
5.812 \\
5.838 \\
7.481 \\
7.908\end{array}$ & 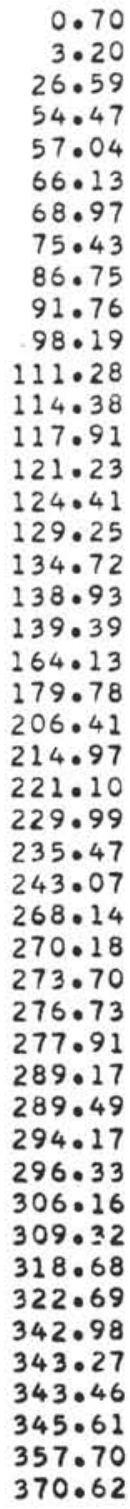 \\
\hline
\end{tabular}


TABLE 9 - Continued

\begin{tabular}{|c|c|c|c|c|c|c|c|c|c|c|c|}
\hline $\begin{array}{c}\text { DATE } \\
(\text { day/mo) }\end{array}$ & $\underset{(\mathrm{hr} / \mathrm{min})}{\mathrm{GMT}}$ & $\begin{array}{l}\text { LONGITUDE } \\
\text { (deg/min) }\end{array}$ & $\begin{array}{l}\text { LATITUDE } \\
\text { (deg/min) }\end{array}$ & $\begin{array}{c}\text { DATE } \\
(\text { day/mo })\end{array}$ & $\begin{array}{c}\text { GMT } \\
(\mathrm{hr} / \mathrm{min})\end{array}$ & $\begin{array}{l}\text { LONGITUDE } \\
\text { (deg/min) }\end{array}$ & $\begin{array}{l}\text { LATITUDE } \\
\text { (deg/min) }\end{array}$ & $\begin{array}{c}\text { DIST } \\
(n m i)\end{array}$ & $\begin{array}{l}\text { CSE } \\
\left({ }^{\circ}\right)\end{array}$ & $\begin{array}{l}\text { SPEED } \\
(k t)\end{array}$ & $\begin{array}{l}\text { DISTT } \\
\text { (nmi) }\end{array}$ \\
\hline $\begin{array}{l}3107 \\
3107 \\
3107 \\
3107 \\
3107 \\
3107 \\
3107 \\
3107 \\
3107 \\
3107 \\
3107 \\
108 \\
108 \\
108 \\
108 \\
108 \\
108 \\
108 \\
108 \\
108 \\
108 \\
108 \\
108 \\
108 \\
108 \\
108 \\
108 \\
108 \\
108 \\
108 \\
108 \\
208 \\
208 \\
208 \\
208 \\
208 \\
208 \\
208 \\
208 \\
208 \\
208 \\
208 \\
208 \\
208 \\
208\end{array}$ & $\begin{array}{l}13 H O \\
13 H 32 \\
14 H 30 \\
14 H 51 \\
15 H 28 \\
17 H 18 \\
17 H 54 \\
18 H 53 \\
19 H 42 \\
20 H 22 \\
23 H 2 \\
0 H 0 \\
3 H 0 \\
3 H 24 \\
3 H 52 \\
5 H 18 \\
6 H 4 \\
7 H 36 \\
10 H 22 \\
10 H 54 \\
12 H 0 \\
12 H 20 \\
12 H 42 \\
14 H 42 \\
15 H 20 \\
17 H 0 \\
17 H 0 \\
18 H 50 \\
22 H 14 \\
23 H 20 \\
23 H 58 \\
0 H 0 \\
O H 36 \\
1 H 48 \\
4 H 20 \\
5 H 0 \\
6 H 46 \\
9 H 30 \\
9 H 53 \\
10 H 10 \\
10 H 45 \\
11 H 12 \\
11 H 15 \\
11 H 54\end{array}$ & 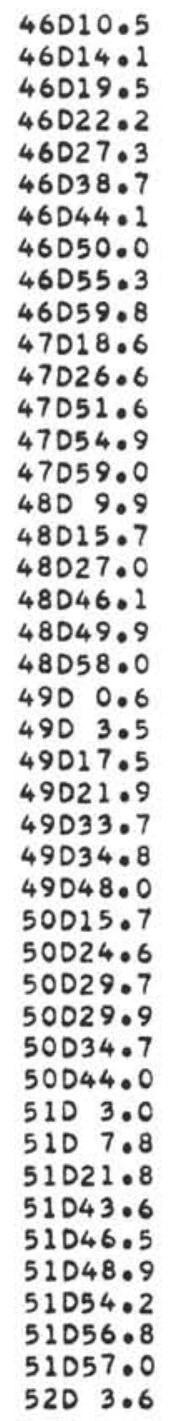 & 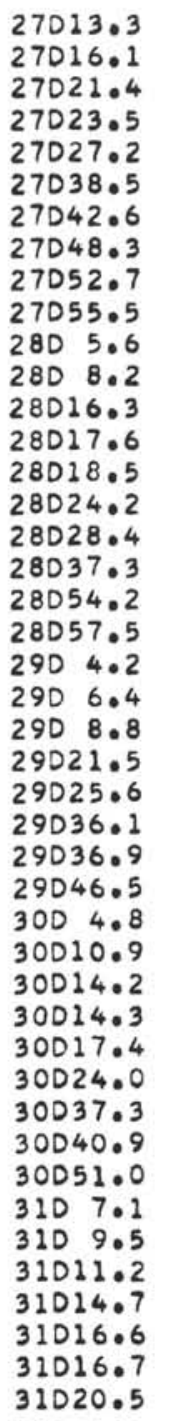 & 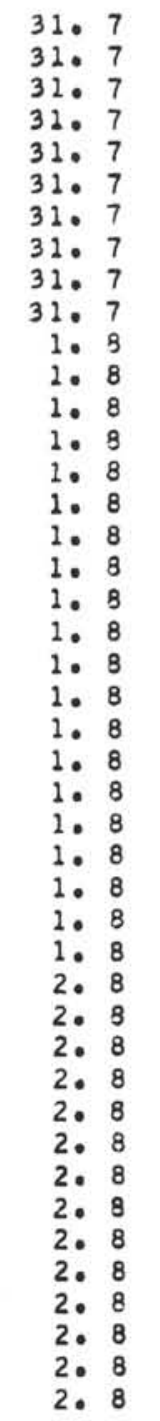 & $\begin{array}{r}13 H 32 \\
14 H 30 \\
14 H 51 \\
15 H 28 \\
17 H 18 \\
17 H 54 \\
18 H 53 \\
19 H 42 \\
20 H 22 \\
23 H \quad 2 \\
0 H 0 \\
3 H 0 \\
3 H 24 \\
3 H 52 \\
5 H 18 \\
6 H 4 \\
7 H 36 \\
10 H 22 \\
10 H 54 \\
12 H 0 \\
12 H 20 \\
12 H 42 \\
14 H 42 \\
15 H 20 \\
17 H 0 \\
17 H 8 \\
18 H 50 \\
22 H 14 \\
23 H 20 \\
23 H 58 \\
0 H 00 \\
0 H 36 \\
1 H 48 \\
4 H 20 \\
5 H 0 \\
6 H 46 \\
9 H 30 \\
9 H 53 \\
10 H 10 \\
10 H 45 \\
11 H 12 \\
11 H 15 \\
11 H 54 \\
16 H 0\end{array}$ & $\begin{array}{l}46014 \cdot 1 \\
46019 \cdot 5 \\
46022 \cdot 2 \\
46027 \cdot 3 \\
46038 \cdot 7 \\
46044 \cdot 1 \\
46050 \cdot 0 \\
46055 \cdot 3 \\
46059 \cdot 8 \\
47018 \cdot 6 \\
47026 \cdot 6 \\
47051 \cdot 6 \\
47054 \cdot 9 \\
47059 \cdot 0 \\
4809 \cdot 9 \\
48015 \cdot 7 \\
48027 \cdot 0 \\
48046 \cdot 1 \\
48049 \cdot 9 \\
48058 \cdot 0 \\
4900 \cdot 6 \\
4903 \cdot 5 \\
49017 \cdot 5 \\
49021 \cdot 9 \\
49033 \cdot 7 \\
49034 \cdot 8 \\
49048 \cdot 0 \\
50015 \cdot 7 \\
50024 \cdot 6 \\
50029 \cdot 7 \\
50029 \cdot 9 \\
50034 \cdot 7 \\
50044 \cdot 0 \\
5103 \cdot 0 \\
5107 \cdot 8 \\
51021 \cdot 8 \\
51043 \cdot 6 \\
51046 \cdot 5 \\
51048 \cdot 9 \\
51054 \cdot 2 \\
51056 \cdot 8 \\
51057 \cdot 0 \\
5203 \cdot 6 \\
52044 \cdot 7\end{array}$ & $\begin{array}{l}27016 \cdot 1 \\
27021 \cdot 4 \\
27023 \cdot 5 \\
27027 \cdot 2 \\
27038 \cdot 5 \\
27042 \cdot 6 \\
27048 \cdot 3 \\
27052 \cdot 7 \\
27055 \cdot 5 \\
2805 \cdot 6 \\
2808 \cdot 2 \\
28016 \cdot 3 \\
28017 \cdot 6 \\
28018 \cdot 5 \\
28024 \cdot 2 \\
28028 \cdot 4 \\
28037 \cdot 3 \\
28054 \cdot 2 \\
28057 \cdot 5 \\
2904 \cdot 2 \\
2906 \cdot 4 \\
2908 \cdot 8 \\
29021 \cdot 5 \\
29025 \cdot 6 \\
29036 \cdot 1 \\
29036 \cdot 9 \\
29046 \cdot 5 \\
3004 \cdot 8 \\
30010 \cdot 9 \\
30014 \cdot 2 \\
30014 \cdot 3 \\
30017 \cdot 4 \\
30024 \cdot 0 \\
30037 \cdot 3 \\
30040 \cdot 9 \\
30051 \cdot 0 \\
3107 \cdot 1 \\
3109 \cdot 5 \\
31011 \cdot 2 \\
31014 \cdot 7 \\
31016 \cdot 6 \\
31016 \cdot 7 \\
31020 \cdot 5 \\
31042 \cdot 5\end{array}$ & $\begin{array}{r}4.23 \\
7.16 \\
3.30 \\
5.76 \\
15.19 \\
6.31 \\
7.82 \\
6.36 \\
4.91 \\
19.46 \\
7.60 \\
23.59 \\
3.13 \\
3.81 \\
11.12 \\
6.66 \\
13.32 \\
23.85 \\
4.69 \\
9.88 \\
3.16 \\
3.40 \\
17.66 \\
5.62 \\
14.80 \\
1.17 \\
14.99 \\
30.28 \\
9.85 \\
5.52 \\
0.28 \\
5.17 \\
10.35 \\
21.15 \\
5.49 \\
15.75 \\
24.73 \\
3.53 \\
2.60 \\
5.86 \\
2.81 \\
0.30 \\
6.71 \\
41.56\end{array}$ & 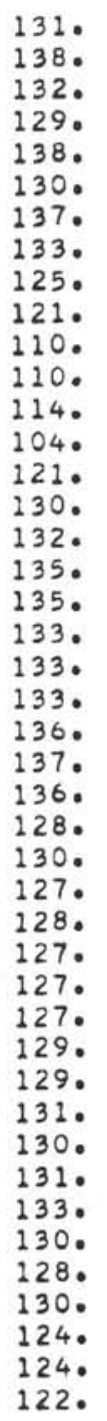 & $\begin{array}{r}7.934 \\
7.413 \\
9.436 \\
9.341 \\
8.289 \\
10.530 \\
7.958 \\
7.798 \\
7.376 \\
7.299 \\
7.865 \\
7.866 \\
7.833 \\
8.176 \\
7.762 \\
8.696 \\
8.693 \\
8.620 \\
8.807 \\
8.983 \\
9.482 \\
9.284 \\
8.832 \\
8.884 \\
8.882 \\
8.796 \\
8.823 \\
8.906 \\
8.956 \\
8.721 \\
8.620 \\
8.620 \\
8.627 \\
8.350 \\
8.240 \\
8.917 \\
9.050 \\
9.213 \\
9.179 \\
10.048 \\
6.254 \\
6.037 \\
10.337 \\
10.138\end{array}$ & 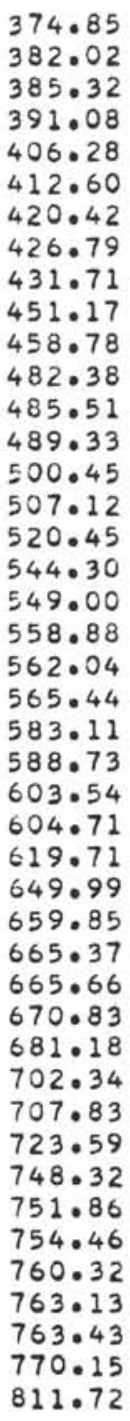 \\
\hline
\end{tabular}


Glomar Challenger, DSDP Leg 25, Navigation Data

\begin{tabular}{|c|c|c|c|c|c|c|c|c|c|c|c|}
\hline $\begin{array}{c}\text { DATE } \\
\text { (day/mo) }\end{array}$ & $\begin{array}{c}\text { GMT } \\
(\mathrm{hr} / \mathrm{min})\end{array}$ & $\begin{array}{l}\text { LONGITUDE } \\
\text { (deg/min) }\end{array}$ & $\begin{array}{l}\text { LATITUDE } \\
\text { (deg/min) }\end{array}$ & $\begin{array}{c}\text { DATE } \\
\text { (day/mo) }\end{array}$ & $\begin{array}{c}\text { GMT } \\
(\mathrm{hr} / \mathrm{min})\end{array}$ & $\begin{array}{l}\text { LONGITUDE } \\
\text { (deg/min) }\end{array}$ & $\begin{array}{l}\text { LATITUDE } \\
\text { (deg/min) }\end{array}$ & $\begin{array}{l}\text { DIST } \\
(\mathrm{nmi})\end{array}$ & $\begin{array}{l}\text { CSE } \\
\left({ }^{\circ}\right)\end{array}$ & $\begin{array}{l}\text { SPEED } \\
(k t)\end{array}$ & $\begin{array}{l}\text { DISTT } \\
(n m i)\end{array}$ \\
\hline 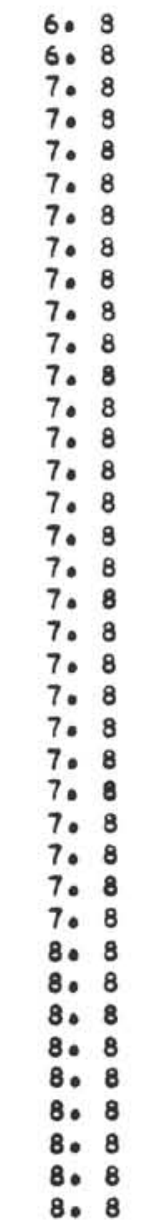 & 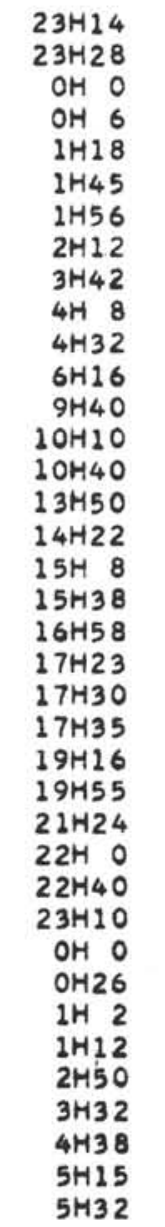 & $\begin{array}{l}52018.1 \\
52017 \cdot 1 \\
52014.9 \\
52014.6 \\
5209.9 \\
5207.4 \\
5206.6 \\
5207.0 \\
51059.2 \\
51057 \cdot 1 \\
51055.6 \\
51047.5 \\
51032.5 \\
51030.5 \\
51028.7 \\
51018.7 \\
51016.9 \\
51013.4 \\
51011.2 \\
5106.1 \\
5103.7 \\
5103.2 \\
5102.8 \\
50056.4 \\
50053.2 \\
50046.8 \\
50043.9 \\
50041.6 \\
50040.0 \\
50037.3 \\
50036.0 \\
50034.3 \\
50033.7 \\
50026.2 \\
50022.6 \\
50016.8 \\
50013.4 \\
50012.0\end{array}$ & 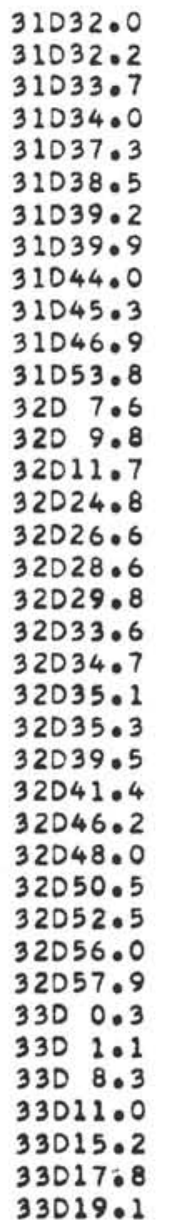 & 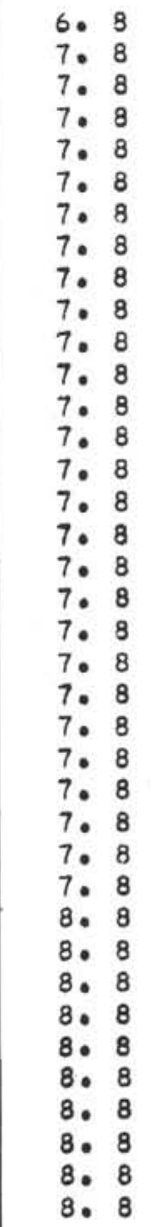 & $\begin{array}{r}23 H 28 \\
O H O \\
O H \\
O H \\
1 H 18 \\
1 H 45 \\
1 H 56 \\
2 H 12 \\
3 H 42 \\
4 H 8 \\
4 H 32 \\
6 H 16 \\
9 H 40 \\
10 H 10 \\
10 H 40 \\
13 H 50 \\
14 H 22 \\
15 H 8 \\
15 H 38 \\
16 H 58 \\
17 H 23 \\
17 H 30 \\
17 H 35 \\
19 H 16 \\
19 H 55 \\
21 H 24 \\
22 H 0 \\
22 H 40 \\
23 H 10 \\
0 H 0 \\
0 H 26 \\
1 H H 2 \\
1 H 12 \\
2 H 50 \\
3 H 32 \\
4 H 38 \\
5 H 15 \\
5 H 32 \\
7 H 58\end{array}$ & 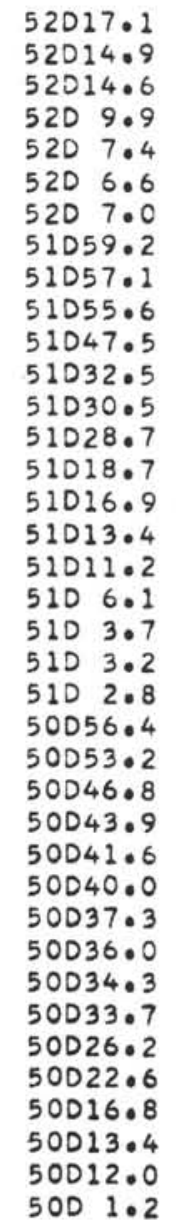 & $\begin{array}{l}31032 \cdot 2 \\
31033 \cdot 7 \\
31034 \cdot 0 \\
31037 \cdot 3 \\
31038 \cdot 5 \\
31039 \cdot 2 \\
31039 \cdot 9 \\
31044 \cdot 0 \\
31045 \cdot 3 \\
31046 \cdot 9 \\
31053 \cdot 8 \\
3207 \cdot 6 \\
3209 \cdot 8 \\
32011 \cdot 7 \\
32024 \cdot 8 \\
32026 \cdot 6 \\
32028 \cdot 6 \\
32029 \cdot 8 \\
32033 \cdot 6 \\
32034 \cdot 7 \\
32035 \cdot 1 \\
32035 \cdot 3 \\
32039 \cdot 5 \\
32041 \cdot 4 \\
32046 \cdot 2 \\
32048 \cdot 0 \\
32050 \cdot 5 \\
32052 \cdot 5 \\
32056 \cdot 0 \\
32057 \cdot 9 \\
3300 \cdot 3 \\
3301 \cdot 1 \\
3308 \cdot 3 \\
33011 \cdot 0 \\
33015 \cdot 2 \\
33017 \cdot 8 \\
33019 \cdot 1 \\
33031 \cdot 1\end{array}$ & $\begin{array}{r}0.87 \\
2.35 \\
0.44 \\
5.20 \\
2.42 \\
0.97 \\
0.77 \\
7.82 \\
2.21 \\
2.02 \\
9.76 \\
18.80 \\
2.78 \\
2.45 \\
15.59 \\
2.40 \\
3.55 \\
2.19 \\
5.76 \\
2.25 \\
0.62 \\
0.36 \\
6.87 \\
3.25 \\
7.27 \\
3.02 \\
3.16 \\
2.41 \\
4.19 \\
2.17 \\
2.79 \\
0.97 \\
9.56 \\
4.07 \\
6.42 \\
3.85 \\
1.75 \\
15.09\end{array}$ & 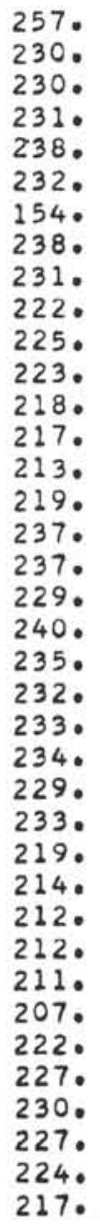 & $\begin{array}{l}3.769 \\
4.416 \\
4.416 \\
4.336 \\
5.396 \\
5.337 \\
2.921 \\
5.218 \\
5.116 \\
5.064 \\
5.635 \\
5.532 \\
5.562 \\
4.900 \\
4.924 \\
4.508 \\
4.534 \\
4.395 \\
4.327 \\
5.416 \\
5.336 \\
4.379 \\
4.086 \\
5.015 \\
4.903 \\
5.035 \\
4.752 \\
4.826 \\
5.028 \\
5.022 \\
4.658 \\
5.864 \\
5.856 \\
5.815 \\
5.839 \\
6.244 \\
6.211 \\
6.202\end{array}$ & $\begin{array}{r}0.87 \\
3.23 \\
3.67 \\
8.87 \\
11.30 \\
12.28 \\
13.06 \\
20.89 \\
23.11 \\
25.13 \\
34.90 \\
53.71 \\
56.49 \\
58.94 \\
74.54 \\
76.94 \\
80.50 \\
82.69 \\
88.46 \\
90.72 \\
91.34 \\
91.71 \\
98.59 \\
101.85 \\
109.12 \\
112.14 \\
115.31 \\
117.72 \\
121.91 \\
124.09 \\
126.89 \\
127.86 \\
137.43 \\
141.50 \\
147.92 \\
151.77 \\
153.53 \\
168.63\end{array}$ \\
\hline
\end{tabular}


TABLE 11

Glomar Challenger, DSDP Leg 25, Navigation Data

\begin{tabular}{|c|c|c|c|c|c|c|c|c|c|c|c|}
\hline $\begin{array}{c}\text { DATE } \\
\text { (day/mo) }\end{array}$ & $\begin{array}{c}\mathrm{GMT} \\
(\mathrm{hr} / \mathrm{min})\end{array}$ & $\begin{array}{l}\text { LONGITUDE } \\
(\mathrm{deg} / \mathrm{min})\end{array}$ & $\begin{array}{l}\text { LATITUDE } \\
(\mathrm{deg} / \mathrm{min})\end{array}$ & $\begin{array}{c}\text { DATE } \\
\text { (day/mo) }\end{array}$ & $\begin{array}{c}\text { GMT } \\
(\mathrm{hr} / \mathrm{min})\end{array}$ & $\begin{array}{l}\text { LONGITUDE } \\
\text { (deg/min) }\end{array}$ & $\begin{array}{l}\text { LATITUDE } \\
\text { (deg/min) }\end{array}$ & $\begin{array}{l}\text { DIST } \\
(\mathrm{nmi})\end{array}$ & $\begin{array}{l}\text { CSE } \\
\left({ }^{\circ}\right)\end{array}$ & $\begin{array}{l}\text { SPEED } \\
(k t)\end{array}$ & $\begin{array}{l}\text { DISTT } \\
(\mathrm{nmi})\end{array}$ \\
\hline 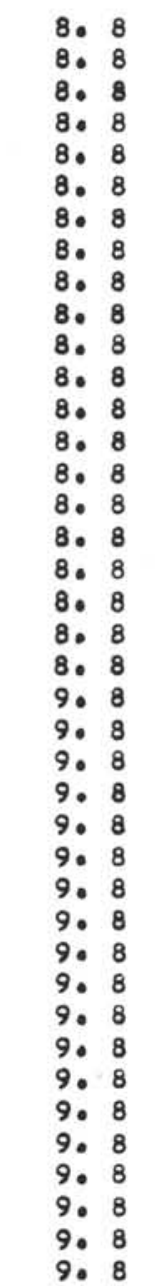 & 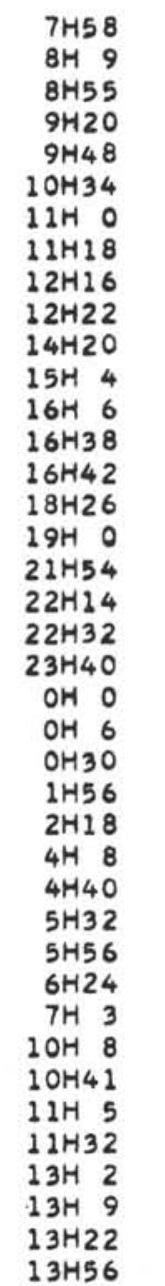 & 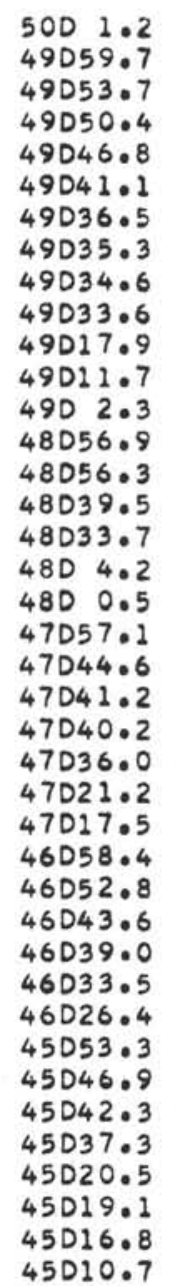 & 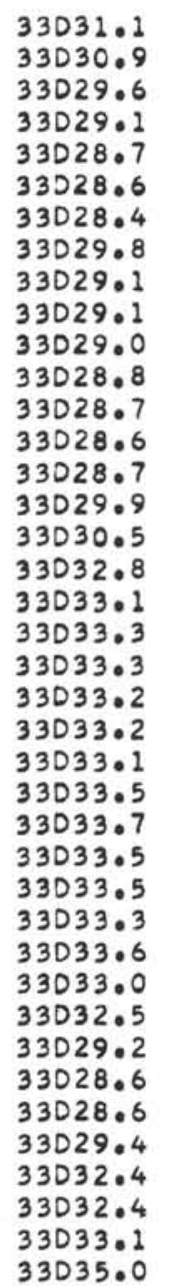 & 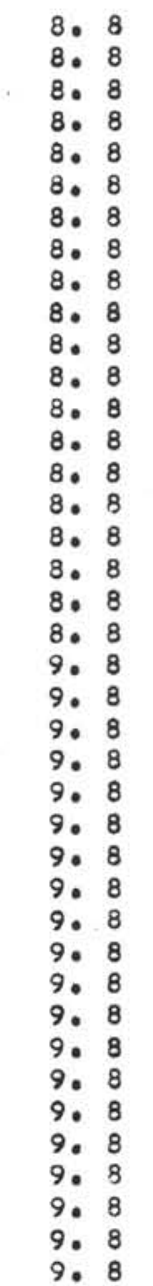 & $\begin{array}{r}8 H 9 \\
8 H 55 \\
9 H 20 \\
9 H 48 \\
10 H 34 \\
11 H O \\
11 H 18 \\
12 H 16 \\
12 H 22 \\
14 H 20 \\
15 H 4 \\
16 H \quad 6 \\
16 H 38 \\
16 H 42 \\
18 H 26 \\
19 H 0 \\
21 H 54 \\
22 H 14 \\
22 H 32 \\
23 H 40 \\
0 H 0 \\
0 H 6 \\
0 H 30 \\
1 H 56 \\
2 H 18 \\
4 H 8 \\
4 H 40 \\
5 H 32 \\
5 H 56 \\
6 H 24 \\
7 H 3 \\
10 H 8 \\
10 H 41 \\
11 H 5 \\
11 H 32 \\
13 H 2 \\
13 H 09 \\
13 H 22 \\
13 H 56 \\
14 H 55\end{array}$ & $\begin{array}{l}49059 \cdot 7 \\
49053 \cdot 7 \\
49050 \cdot 4 \\
49046 \cdot 8 \\
49041 \cdot 1 \\
49036 \cdot 5 \\
49035 \cdot 3 \\
49034 \cdot 6 \\
49033 \cdot 6 \\
49017 \cdot 9 \\
49011 \cdot 7 \\
4902 \cdot 3 \\
48056 \cdot 9 \\
48056 \cdot 3 \\
48039 \cdot 5 \\
48033 \cdot 7 \\
4804 \cdot 2 \\
4800 \cdot 5 \\
47057 \cdot 1 \\
47044 \cdot 6 \\
47041 \cdot 2 \\
47040 \cdot 2 \\
47036 \cdot 0 \\
47021 \cdot 2 \\
47017 \cdot 5 \\
46058 \cdot 4 \\
46052 \cdot 8 \\
46043 \cdot 6 \\
46039 \cdot 0 \\
46033 \cdot 5 \\
46026 \cdot 4 \\
45053 \cdot 3 \\
45046 \cdot 9 \\
45042 \cdot 3 \\
45037 \cdot 3 \\
45020 \cdot 5 \\
45019 \cdot 1 \\
45016 \cdot 8 \\
45010 \cdot 7 \\
4501 \cdot 5\end{array}$ & $\begin{array}{l}33030 \cdot 9 \\
33029 \cdot 6 \\
33029 \cdot 1 \\
33028 \cdot 7 \\
33028 \cdot 6 \\
33028 \cdot 4 \\
33029 \cdot 8 \\
33029 \cdot 1 \\
33029 \cdot 1 \\
33029 \cdot 0 \\
33028 \cdot 8 \\
33028 \cdot 7 \\
33028 \cdot 6 \\
33028 \cdot 7 \\
33029 \cdot 9 \\
33030 \cdot 5 \\
33032 \cdot 8 \\
33033 \cdot 1 \\
33033 \cdot 3 \\
33033 \cdot 3 \\
33033 \cdot 2 \\
33033 \cdot 2 \\
33033 \cdot 1 \\
33033 \cdot 5 \\
33033 \cdot 7 \\
33033 \cdot 5 \\
33033 \cdot 5 \\
33033 \cdot 3 \\
33033 \cdot 6 \\
33033 \cdot 0 \\
33032 \cdot 5 \\
33029 \cdot 2 \\
33028 \cdot 6 \\
33028 \cdot 6 \\
33029 \cdot 4 \\
33032 \cdot 4 \\
33032 \cdot 4 \\
33033 \cdot 1 \\
33035 \cdot 0 \\
33038 \cdot 9\end{array}$ & $\begin{array}{r}1.24 \\
5.25 \\
2.81 \\
2.98 \\
4.83 \\
3.78 \\
1.71 \\
0.88 \\
0.89 \\
13.15 \\
5.13 \\
7.94 \\
4.47 \\
0.55 \\
14.12 \\
4.86 \\
24.85 \\
3.11 \\
2.79 \\
10.53 \\
2.83 \\
0.85 \\
3.47 \\
12.44 \\
3.10 \\
15.99 \\
4.66 \\
7.70 \\
3.86 \\
4.64 \\
5.88 \\
28.00 \\
5.35 \\
3.86 \\
4.27 \\
14.38 \\
1.12 \\
2.07 \\
5.44 \\
8.63\end{array}$ & 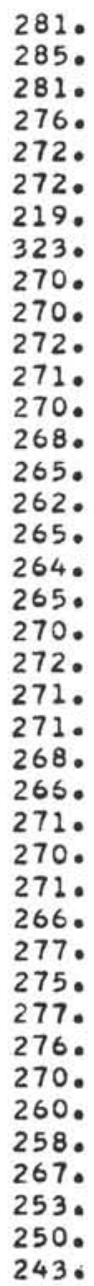 & $\begin{array}{l}6.809 \\
6.858 \\
6.760 \\
6.391 \\
6.301 \\
8.745 \\
5.705 \\
0.915 \\
8.992 \\
6.689 \\
7.000 \\
7.686 \\
8.383 \\
8.351 \\
8.156 \\
8.579 \\
8.569 \\
9.337 \\
9.302 \\
9.293 \\
8.504 \\
8.505 \\
8.677 \\
8.680 \\
8.465 \\
8.722 \\
8.749 \\
8.890 \\
9.657 \\
9.951 \\
9.052 \\
9.082 \\
9.735 \\
9.651 \\
9.501 \\
9.591 \\
9.654 \\
9.566 \\
9.615 \\
8.778\end{array}$ & $\begin{array}{r}1.24 \\
6.50 \\
9.32 \\
12.30 \\
17.13 \\
20.92 \\
22.63 \\
23.52 \\
24.42 \\
37.57 \\
42.71 \\
50.65 \\
55.12 \\
55.58 \\
59.81 \\
74.67 \\
99.52 \\
102.63 \\
105.43 \\
115.96 \\
118.79 \\
119.64 \\
123.12 \\
135.56 \\
138.66 \\
154.65 \\
159.32 \\
167.03 \\
170.89 \\
175053 \\
181.42 \\
209.42 \\
214.78 \\
218.64 \\
222.91 \\
237.30 \\
238.43 \\
240.50 \\
245.95 \\
254.58\end{array}$ \\
\hline
\end{tabular}


TABLE 12

Glomar Challenger, DSDP Leg 25, Navigation Data

\begin{tabular}{|c|c|c|c|c|c|c|c|c|c|c|c|}
\hline $\begin{array}{c}\text { DATE } \\
\text { (day/mo) }\end{array}$ & $\begin{array}{c}\text { GMT } \\
(\mathrm{hr} / \mathrm{min})\end{array}$ & $\begin{array}{l}\text { LONGITUDE } \\
\text { (deg/min) }\end{array}$ & $\begin{array}{r}\text { LATITUDE } \\
\text { (deg/min) }\end{array}$ & $\begin{array}{c}\text { DATE } \\
\text { (day/mo) }\end{array}$ & $\begin{array}{c}\text { GMT } \\
(\mathrm{hr} / \mathrm{min})\end{array}$ & $\begin{array}{l}\text { LONGITUDE } \\
\text { (deg/min) }\end{array}$ & $\begin{array}{l}\text { LATITUDE } \\
\text { (deg/min) }\end{array}$ & $\begin{array}{c}\text { DIST } \\
(\mathrm{nmi})\end{array}$ & $\begin{array}{l}\text { CSE } \\
\left({ }^{\circ}\right)\end{array}$ & $\begin{array}{l}\text { SPEED } \\
(k t)\end{array}$ & $\begin{array}{l}\text { DISTT } \\
(\mathrm{nmi})\end{array}$ \\
\hline $\begin{array}{l}10 \cdot 8 \\
10 \cdot 8 \\
10 \cdot 8 \\
10.8 \\
10.8\end{array}$ & $\begin{array}{l}14 \mathrm{H} 20 \\
14 \mathrm{H} 38 \\
14 \mathrm{H} 52 \\
15 \mathrm{H} 18 \\
16 \mathrm{H} 20\end{array}$ & 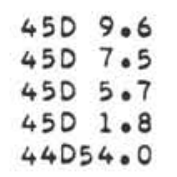 & $\begin{array}{l}33037 \cdot 2 \\
33037 \cdot 2 \\
33037 \cdot 2 \\
33037 \cdot 3 \\
33037.6\end{array}$ & $\begin{array}{ll}10 . & 8 \\
10 \cdot & 8 \\
10 . & 8 \\
10 . & 8 \\
10 . & 8\end{array}$ & $\begin{array}{l}14 H 38 \\
14 H 52 \\
15 H 18 \\
16 H 20 \\
16 H 29\end{array}$ & $\begin{array}{l}4507.5 \\
4505.7 \\
4501.8 \\
44 D 54.0 \\
44052.6\end{array}$ & $\begin{array}{l}33037 \cdot 2 \\
33037 \cdot 2 \\
33037 \cdot 3 \\
33037 \cdot 6 \\
33037.7\end{array}$ & $\begin{array}{l}1.70 \\
1.55 \\
3.19 \\
6.59 \\
1.17\end{array}$ & $\begin{array}{l}269^{\circ} \\
271^{\circ} \\
268^{\circ} \\
268^{\circ} \\
264^{\circ}\end{array}$ & $\begin{array}{l}5.692 \\
6.661 \\
7.384 \\
6.383 \\
7.847\end{array}$ & $\begin{array}{r}1.70 \\
3.26 \\
6.46 \\
13.05 \\
14.23\end{array}$ \\
\hline
\end{tabular}


TABLE 13

Glomar Challenger, DSDP Leg 25, Navigation Data

\begin{tabular}{|c|c|c|c|c|c|c|c|c|c|c|c|}
\hline $\begin{array}{c}\text { DATE } \\
\text { (day/mo) }\end{array}$ & $\begin{array}{c}\text { GMT } \\
(\mathrm{hr} / \mathrm{min})\end{array}$ & $\begin{array}{l}\text { LONGITUDE } \\
\text { (deg/min) }\end{array}$ & $\begin{array}{r}\text { LATITUDE } \\
\text { (deg/min) }\end{array}$ & $\begin{array}{c}\text { DATE } \\
(\text { day } / \mathrm{mo})\end{array}$ & $\begin{array}{c}\text { GMT } \\
(\mathrm{hr} / \mathrm{min})\end{array}$ & $\begin{array}{l}\text { LONGITUDE } \\
(\mathrm{deg} / \mathrm{min})\end{array}$ & $\begin{array}{l}\text { LATITUDE } \\
\text { (deg/min) }\end{array}$ & $\begin{array}{l}\text { DIST } \\
(\mathrm{nmi})\end{array}$ & $\begin{array}{l}\text { CSE } \\
\left({ }^{\circ}\right)\end{array}$ & $\begin{array}{l}\text { SPEED } \\
(k t)\end{array}$ & $\begin{array}{l}\text { DISTT. } \\
\text { (nmi) }\end{array}$ \\
\hline $\begin{array}{l}1108 \\
1108 \\
1108 \\
1108 \\
1108 \\
1208 \\
1108 \\
1108 \\
1108 \\
1108 \\
1108 \\
1108 \\
1108 \\
1108 \\
1108 \\
1108 \\
1208 \\
1208 \\
1208 \\
1208 \\
1208 \\
1208 \\
1208 \\
1208 \\
1208 \\
1208 \\
1208 \\
1208 \\
1208 \\
1208 \\
1208 \\
1208 \\
1208 \\
1308 \\
1308 \\
1308 \\
1308 \\
1308 \\
1308 \\
1308 \\
1308 \\
1308 \\
1308 \\
1308\end{array}$ & 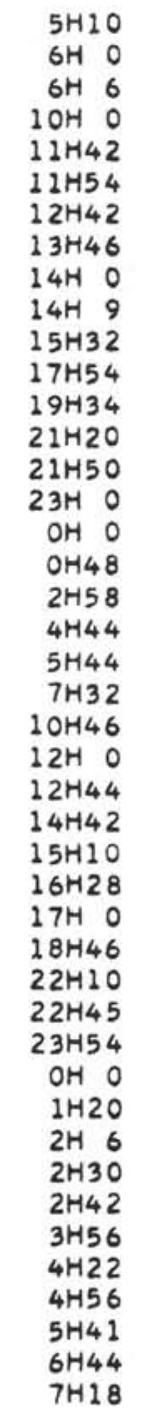 & 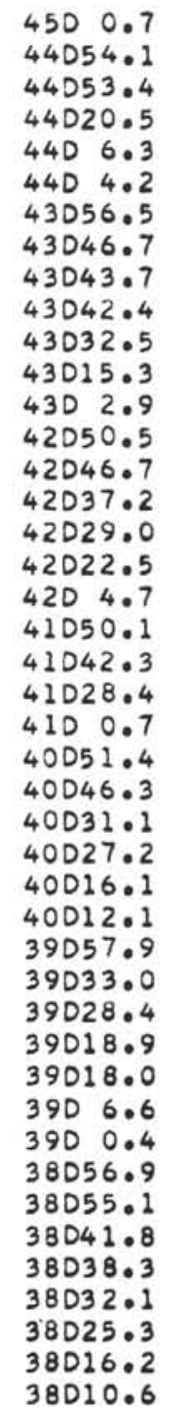 & 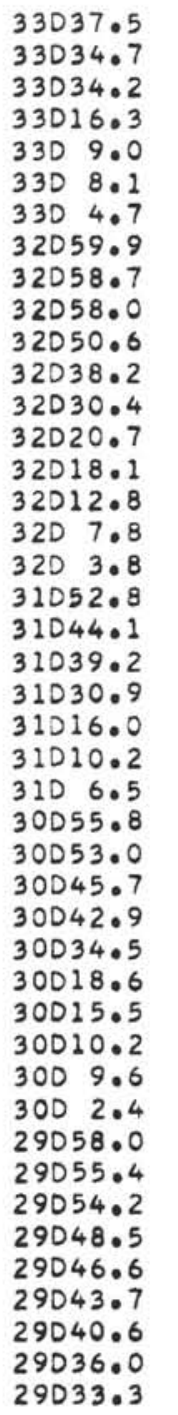 & 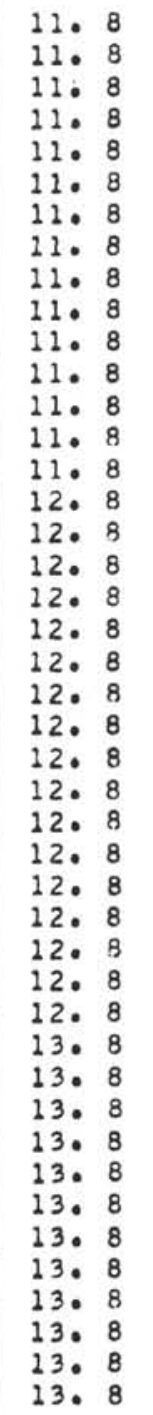 & $\begin{array}{r}6 H O \\
6 H \quad 6 \\
10 H 0 \\
11 H 42 \\
11 H 54 \\
112 H 42 \\
12 H 46 \\
13 H 46 \\
14 H 0 \\
14 H 0 \\
15 H 32 \\
17 H 54 \\
19 H 34 \\
21 H 20 \\
21 H 50 \\
23 H 0 \\
0 H 0 \\
0 H 48 \\
2 H 58 \\
4 H 44 \\
5 H 44 \\
7 H 32 \\
10 H 46 \\
12 H 0 \\
12 H 44 \\
14 H 42 \\
15 H 10 \\
16 H 28 \\
17 H 0 \\
18 H 46 \\
22 H 10 \\
22 H 45 \\
23 H 54 \\
0 H 0 \\
1 H 20 \\
2 H 06 \\
2 H 30 \\
2 H 42 \\
3 H 56 \\
4 H 22 \\
4 H 56 \\
5 H 41 \\
6 H 44 \\
7 H 18 \\
8 H 35\end{array}$ & $\begin{array}{l}44 D 54 \cdot 1 \\
44 D 53 \cdot 4 \\
44020 \cdot 5 \\
4406 \cdot 3 \\
4404 \cdot 2 \\
43056 \cdot 5 \\
43046 \cdot 7 \\
43043 \cdot 7 \\
43042 \cdot 4 \\
43032 \cdot 5 \\
43015 \cdot 3 \\
4302 \cdot 9 \\
42050 \cdot 5 \\
42046 \cdot 7 \\
42037 \cdot 2 \\
42029 \cdot 0 \\
42022 \cdot 5 \\
4204 \cdot 7 \\
41050 \cdot 1 \\
41042 \cdot 3 \\
41028 \cdot 4 \\
4100 \cdot 7 \\
40051 \cdot 4 \\
40046 \cdot 3 \\
40031 \cdot 1 \\
40027 \cdot 2 \\
40016 \cdot 1 \\
40012 \cdot 1 \\
39057 \cdot 9 \\
39033 \cdot 0 \\
39028 \cdot 4 \\
39018 \cdot 9 \\
39018 \cdot 0 \\
3906 \cdot 6 \\
3900 \cdot 4 \\
38056 \cdot 9 \\
38055 \cdot 1 \\
38041 \cdot 8 \\
38038 \cdot 3 \\
38032 \cdot 1 \\
38025 \cdot 3 \\
38016 \cdot 2 \\
38010 \cdot 6 \\
37058 \cdot 9\end{array}$ & $\begin{array}{l}33034 \cdot 7 \\
33034 \cdot 2 \\
33016 \cdot 3 \\
3309 \cdot 0 \\
3308 \cdot 1 \\
3304 \cdot 7 \\
32059 \cdot 9 \\
32058 \cdot 7 \\
32058 \cdot 0 \\
32050 \cdot 6 \\
32038 \cdot 2 \\
32030 \cdot 4 \\
32020 \cdot 7 \\
32018 \cdot 1 \\
32012 \cdot 8 \\
3207 \cdot 8 \\
3203 \cdot 8 \\
31052 \cdot 8 \\
31044 \cdot 1 \\
31039 \cdot 2 \\
31030 \cdot 9 \\
31016 \cdot 0 \\
31010 \cdot 2 \\
3106 \cdot 5 \\
30055 \cdot 8 \\
30053 \cdot 0 \\
30045 \cdot 7 \\
30042 \cdot 9 \\
30034 \cdot 5 \\
30018 \cdot 6 \\
30015 \cdot 5 \\
30010 \cdot 2 \\
3009 \cdot 6 \\
3002 \cdot 4 \\
29058 \cdot 0 \\
29055 \cdot 4 \\
29054 \cdot 2 \\
29048 \cdot 5 \\
29046 \cdot 6 \\
29043 \cdot 7 \\
29040 \cdot 6 \\
29036 \cdot 0 \\
29033 \cdot 3 \\
29026 \cdot 3\end{array}$ & $\begin{array}{r}6.11 \\
0.83 \\
32.88 \\
13.99 \\
1.98 \\
7.25 \\
9.61 \\
2.79 \\
1023 \\
11.22 \\
19.10 \\
13.08 \\
14.30 \\
4.10 \\
9.69 \\
8.56 \\
6.84 \\
18.74 \\
15.20 \\
8.27 \\
14.50 \\
28.04 \\
9.80 \\
5.80 \\
16.90 \\
4.32 \\
12.09 \\
4.44 \\
14.87 \\
26.80 \\
4.999 \\
9.86 \\
0.91 \\
12.23 \\
7.04 \\
3.99 \\
1.999 \\
12.91 \\
3.059 \\
6.13 \\
6.64 \\
9.24 \\
5.553 \\
12.37\end{array}$ & 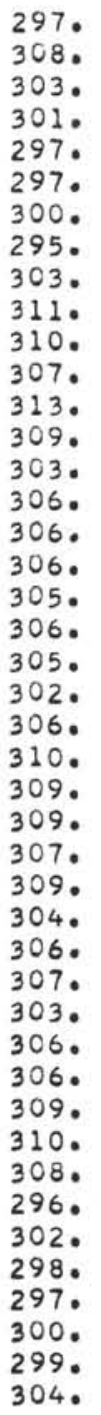 & $\begin{array}{r}7.341 \\
8.384 \\
8.432 \\
8.230 \\
9.913 \\
9.066 \\
9.014 \\
11.994 \\
8.200 \\
8.114 \\
8.073 \\
7.848 \\
8.098 \\
8.216 \\
8.308 \\
8.567 \\
8.552 \\
8.549 \\
8.605 \\
8.275 \\
8.059 \\
8.674 \\
7.952 \\
7.914 \\
8.597 \\
9.275 \\
9.300 \\
8.338 \\
8.420 \\
7.882 \\
8.556 \\
8.579 \\
9.177 \\
9.177 \\
9.190 \\
9.975 \\
9.953 \\
10.474 \\
8.298 \\
10.832 \\
8.856 \\
8.801 \\
9.773 \\
9.643\end{array}$ & $\begin{array}{r}6.11 \\
6.95 \\
39.84 \\
53.83 \\
55.81 \\
63.07 \\
72.68 \\
75.48 \\
76.71 \\
87.94 \\
107.04 \\
120.12 \\
134.43 \\
138.54 \\
148.23 \\
156.80 \\
163.64 \\
182.38 \\
197.59 \\
205.86 \\
220.37 \\
248.42 \\
258.23 \\
264.03 \\
280.94 \\
285.27 \\
297.36 \\
301.80 \\
316.68 \\
343.48 \\
348.47 \\
358.34 \\
359.26 \\
371.49 \\
378.54 \\
382.53 \\
384.552 \\
397.44 \\
401.03 \\
407.17 \\
413.82 \\
423.06 \\
428.60 \\
440.97\end{array}$ \\
\hline
\end{tabular}




\begin{tabular}{|c|c|c|c|c|c|c|c|c|c|c|c|}
\hline $\begin{array}{c}\text { DATE } \\
\text { (day/mo) }\end{array}$ & $\begin{array}{c}\text { GMT } \\
(\mathrm{hr} / \mathrm{min})\end{array}$ & $\begin{array}{l}\text { LONGITUDE } \\
\text { (deg/min) }\end{array}$ & $\begin{array}{l}\text { LATITUDE } \\
\text { (deg/min) }\end{array}$ & $\begin{array}{c}\text { DATE } \\
\text { (day/mo) }\end{array}$ & $\begin{array}{c}\text { GMT } \\
(\mathrm{hr} / \mathrm{min})\end{array}$ & $\begin{array}{l}\text { LONGITUDE } \\
\text { (deg/min) }\end{array}$ & $\begin{array}{l}\text { LATITUDE } \\
\text { (deg/min) }\end{array}$ & $\begin{array}{r}\text { DIST } \\
(\mathrm{nmi})\end{array}$ & $\begin{array}{l}\text { CSE } \\
\left({ }^{\circ}\right)\end{array}$ & $\begin{array}{l}\text { SPEED } \\
(k t)\end{array}$ & $\begin{array}{l}\text { DISTT } \\
(n m i)\end{array}$ \\
\hline $\begin{array}{ll}130 & 8 \\
130 & 8 \\
130 & 8 \\
130 & 8 \\
130 & 8 \\
130 & 8\end{array}$ & $\begin{array}{r}8 H 35 \\
9 H 12 \\
9 H 47 \\
10 H \quad 4 \\
10 H 40 \\
10 H 54\end{array}$ & $\begin{array}{l}37058.9 \\
37051.7 \\
37044.7 \\
37041.3 \\
37033.8 \\
37031.0\end{array}$ & $\begin{array}{l}29026.3 \\
29028.5 \\
29029.6 \\
29030.0 \\
29029.9 \\
29030.4\end{array}$ & $\begin{array}{l}13 \cdot 8 \\
13 \cdot 8 \\
1308 \\
1308 \\
13 \cdot 8 \\
13 \cdot 8\end{array}$ & $\begin{array}{r}9 H 12 \\
9 H 47 \\
10 H \quad 4 \\
10 H 40 \\
10 H 54 \\
13 H 50\end{array}$ & $\begin{array}{l}37051.7 \\
37044.7 \\
37041.3 \\
37033.8 \\
37031.0 \\
3702.4\end{array}$ & $\begin{array}{l}29028.5 \\
29029.6 \\
29030.0 \\
29029.9 \\
29030.4 \\
29035.8\end{array}$ & $\begin{array}{r}6.67 \\
6.23 \\
3.01 \\
6.49 \\
2.50 \\
25.57\end{array}$ & $\begin{array}{l}251^{\circ} \\
260^{\circ} \\
264^{\circ} \\
271^{\circ} \\
2570^{\circ} \\
258^{\circ}\end{array}$ & $\begin{array}{r}10.821 \\
10.683 \\
10.637 \\
10.829 \\
10.717 \\
8.719\end{array}$ & $\begin{array}{r}6.67 \\
12.90 \\
15.91 \\
22.41 \\
24.91 \\
50.49\end{array}$ \\
\hline
\end{tabular}

TABLE 15

Glomar Challenger, DSDP Leg 25, Navigation Data

\begin{tabular}{|c|c|c|c|c|c|c|c|c|c|c|c|}
\hline $\begin{array}{c}\text { DATE } \\
\text { (day/mo) }\end{array}$ & $\begin{array}{c}\text { GMT } \\
(\mathrm{hr} / \mathrm{min})\end{array}$ & $\begin{array}{l}\text { LONGITUDE } \\
\text { (deg/min) }\end{array}$ & $\begin{array}{l}\text { LATITUDE } \\
(\mathrm{deg} / \mathrm{min})\end{array}$ & $\begin{array}{c}\text { DATE } \\
(\text { day/mo })\end{array}$ & $\begin{array}{c}\text { GMT } \\
(\mathrm{hr} / \mathrm{min})\end{array}$ & $\begin{array}{l}\text { LONGITUDE } \\
\text { (deg/min) }\end{array}$ & $\begin{array}{l}\text { LATITUDE } \\
\text { (deg/min) }\end{array}$ & $\begin{array}{l}\text { DIST } \\
(\mathrm{nmi})\end{array}$ & $\begin{array}{l}\text { CSE } \\
\left({ }^{\circ}\right)\end{array}$ & $\begin{array}{l}\text { SPEED } \\
(k t)\end{array}$ & $\begin{array}{l}\text { DISTT } \\
(\mathrm{nmi})\end{array}$ \\
\hline $\begin{array}{l}17 \cdot 8 \\
1708 \\
1708 \\
1708 \\
1708 \\
1708 \\
1708 \\
1708 \\
1708 \\
1708 \\
1708 \\
1708 \\
1708 \\
1708 \\
1708 \\
17 \cdot 8\end{array}$ & $\begin{array}{r}2 H 57 \\
3 H 45 \\
4 H 2 \\
4 H 26 \\
4 H 44 \\
5 H 16 \\
5 H 34 \\
6 H 58 \\
8 H 0 \\
9 H 20 \\
9 H 56 \\
10 H 16 \\
10 H 28 \\
10 H 50 \\
11 H 18 \\
11 H 38\end{array}$ & $\begin{array}{l}37028 \cdot 5 \\
37020 \cdot 3 \\
37016 \cdot 9 \\
37012 \cdot 3 \\
3708 \cdot 8 \\
3702 \cdot 6 \\
36058 \cdot 8 \\
36041 \cdot 0 \\
36028 \cdot 3 \\
36018 \cdot 2 \\
36013 \cdot 8 \\
36011 \cdot 3 \\
36010.0 \\
3607 \cdot 1 \\
3603 \cdot 7 \\
360: 1.5\end{array}$ & $\begin{array}{l}29031.8 \\
29034.6 \\
29035.9 \\
29037.5 \\
29038.4 \\
29039.3 \\
29040.1 \\
29043.6 \\
29045.8 \\
29049.6 \\
29051.5 \\
29052.6 \\
29053.4 \\
29054.7 \\
29056.7 \\
29057.9\end{array}$ & $\begin{array}{l}17 \cdot 8 \\
17: 8 \\
17: 8 \\
17: 8 \\
17: 8 \\
17: 8 \\
17: 8 \\
17: 8 \\
1708 \\
17 \cdot 8 \\
17: 8 \\
17: 8 \\
17: 8 \\
17: 8 \\
17: 8 \\
17: 8\end{array}$ & $\begin{array}{l}3 H 45 \\
4 H 2 \\
4 H 26 \\
4 H 44 \\
5 H 16 \\
5 H 34 \\
6 H 58 \\
8 H 0 \\
9 H 20 \\
9 H 56 \\
1 \mathrm{CH} 16 \\
1 \mathrm{CH} 28 \\
1 \mathrm{CH} 50 \\
12 \mathrm{H} 18 \\
11 \mathrm{H} 38 \\
12 \mathrm{H} 52\end{array}$ & 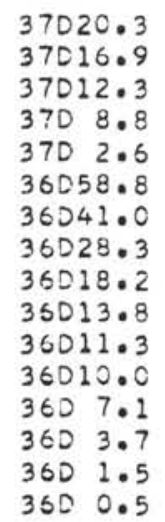 & $\begin{array}{l}29034.6 \\
29035.9 \\
29037.5 \\
29038.4 \\
29039.3 \\
29040.1 \\
29043.6 \\
29045.8 \\
29049.6 \\
29051.5 \\
29052.6 \\
29053.4 \\
29054.7 \\
29056.7 \\
29057.9 \\
29059.1\end{array}$ & $\begin{array}{r}7.63 \\
3.30 \\
4.29 \\
3.23 \\
5.49 \\
3.41 \\
15.93 \\
11.26 \\
9.60 \\
4.28 \\
2.39 \\
1.42 \\
2.81 \\
3.55 \\
2.30 \\
1.46\end{array}$ & $\begin{array}{l}2488^{\circ} \\
2470^{\circ} \\
247 \circ^{\circ} \\
255^{\circ} \\
2610^{\circ} \\
2560^{\circ} \\
257^{\circ} \\
258^{\circ} \\
2470^{\circ} \\
2440^{\circ} \\
2420^{\circ} \\
238^{\circ} \\
240^{\circ} \\
237^{\circ} \\
239^{\circ} \\
2130^{\circ}\end{array}$ & $\begin{array}{r}9.546 \\
11.652 \\
10.740 \\
10.769 \\
10.294 \\
11.376 \\
11.379 \\
10.905 \\
7.201 \\
7.136 \\
7.179 \\
7.133 \\
7.668 \\
7.609 \\
6.910 \\
6.265\end{array}$ & $\begin{array}{l}7.63 \\
10.93 \\
15.23 \\
18.46 \\
23.95 \\
27.36 \\
43.30 \\
54.56 \\
64.17 \\
68.45 \\
70.84 \\
72.27 \\
75.08 \\
78.63 \\
80.94 \\
82.40\end{array}$ \\
\hline
\end{tabular}


TABLE 16

Glomar Challenger, DSDP Leg 25, Navigation Data

\begin{tabular}{|c|c|c|c|c|c|c|c|c|c|c|c|}
\hline $\begin{array}{c}\text { DATE } \\
(\text { day/mo })\end{array}$ & $\begin{array}{c}\text { GMT } \\
(\mathrm{hr} / \mathrm{min})\end{array}$ & $\begin{array}{l}\text { LONGITUDE } \\
(\mathrm{deg} / \mathrm{min})\end{array}$ & $\begin{array}{l}\text { LATITUDE } \\
\text { (deg/min) }\end{array}$ & $\begin{array}{c}\text { DATE } \\
\text { (day/mo) }\end{array}$ & $\begin{array}{c}\text { GMT } \\
(\mathrm{hr} / \mathrm{min})\end{array}$ & $\begin{array}{l}\text { LONGITUDE } \\
\text { (deg/min) }\end{array}$ & $\begin{array}{l}\text { LATITUDE } \\
\text { (deg/min) }\end{array}$ & $\begin{array}{l}\text { DIST } \\
(\mathrm{nmi})\end{array}$ & $\begin{array}{l}\text { CSE } \\
\left({ }^{\circ}\right)\end{array}$ & $\begin{array}{l}\text { SPEED } \\
(k t)\end{array}$ & $\begin{array}{l}\text { DISTT } \\
(\mathrm{nmi})\end{array}$ \\
\hline 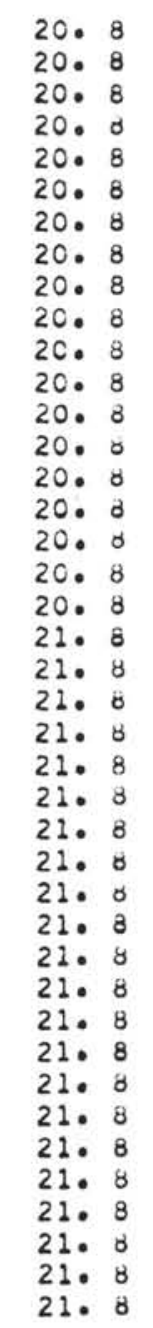 & 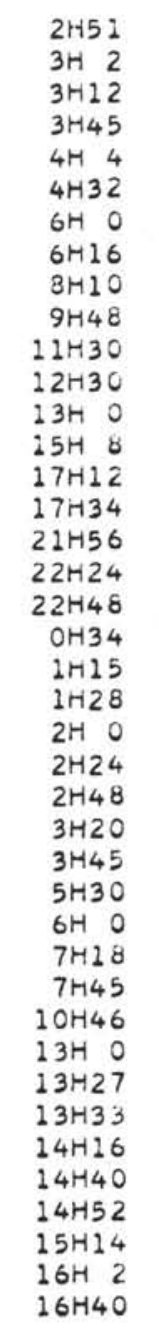 & $\begin{array}{l}3604 \cdot 6 \\
3603 \cdot 0 \\
3601 \cdot 9 \\
35057 \cdot 5 \\
35055 \cdot 0 \\
35051 \cdot 4 \\
35045 \cdot 1 \\
35044 \cdot 0 \\
35024 \cdot 4 \\
35012 \cdot 8 \\
3503 \cdot 0 \\
34054 \cdot 6 \\
34050 \cdot 4 \\
34032 \cdot 6 \\
34019 \cdot 1 \\
34016 \cdot 6 \\
33045 \cdot 5 \\
33042 \cdot 3 \\
33039 \cdot 7 \\
33026 \cdot 3 \\
33021 \cdot 2 \\
33019 \cdot 6 \\
33016 \cdot 1 \\
33013 \cdot 5 \\
33010 \cdot 3 \\
3306 \cdot 2 \\
3303 \cdot 3 \\
32051 \cdot 8 \\
32048 \cdot 6 \\
32040 \cdot 2 \\
32037 \cdot 3 \\
32018 \cdot 2 \\
3202 \cdot 7 \\
3200 \cdot 5 \\
3200 \cdot 1 \\
31056 \cdot 7 \\
31056 \cdot 0 \\
31055 \cdot 0 \\
31053 \cdot 7 \\
31050 \cdot 9 \\
31047 \cdot 9\end{array}$ & 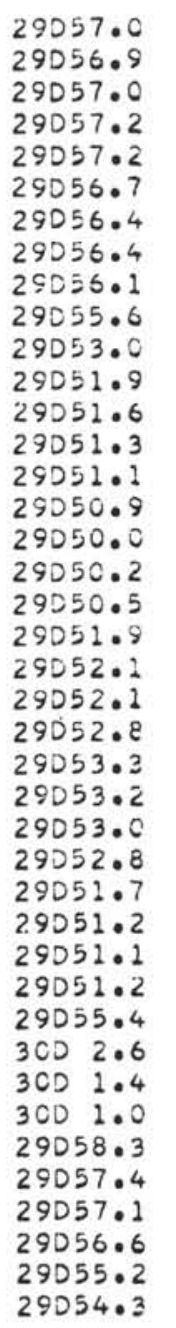 & 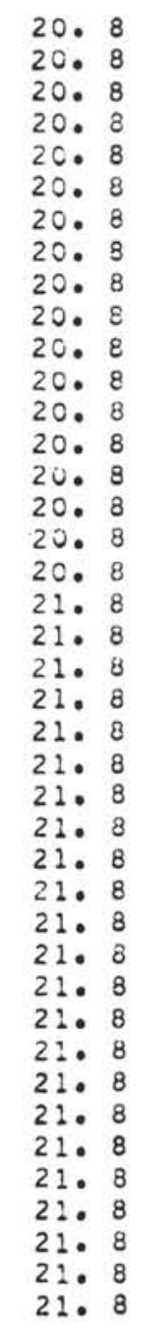 & $\begin{array}{l}3 H 2 \\
3 H 12 \\
3 H 45 \\
4 H \quad 4 \\
4 H 32 \\
6 H O \\
6 H 16 \\
3 H 10 \\
9 H 48 \\
11 H 30 \\
12 H 30 \\
13 H O \\
15 H 8 \\
17 H 12 \\
17 H 34 \\
22 H 56 \\
22 H 24 \\
22 H 48 \\
2 H 34 \\
1 H 15 \\
1 H 28 \\
2 H H O \\
2 H 24 \\
2 H 48 \\
3 H 2 O \\
3 H 45 \\
5 H 30 \\
6 H 0 \\
7 H 18 \\
7 H 45 \\
10 H 46 \\
13 H 0 \\
13 H 27 \\
13 H 33 \\
14 H 16 \\
14 H 40 \\
14 H 52 \\
15 H 14 \\
16 H 2 \\
16 H 40 \\
16 H 44\end{array}$ & $\begin{array}{l}3603 \cdot 0 \\
3601.9 \\
35057 \cdot 5 \\
35055 \cdot 0 \\
35051 \cdot 4 \\
35045 \cdot 1 \\
35244 \cdot 0 \\
35024 \cdot 4 \\
35012 \cdot 9 \\
3503 \cdot 0 \\
34054 \cdot 6 \\
34050 \cdot 4 \\
34032 \cdot 6 \\
34019 \cdot 1 \\
34216 \cdot 6 \\
33045 \cdot 5 \\
33042 \cdot 3 \\
33039 \cdot 7 \\
33026 \cdot 3 \\
32021 \cdot 2 \\
33019 \cdot 6 \\
33016 \cdot 1 \\
33013 \cdot 5 \\
33010 \cdot 3 \\
3300 \cdot 2 \\
3303 \cdot 3 \\
32051 \cdot 8 \\
32048 \cdot 6 \\
32040 \cdot 2 \\
32037 \cdot 3 \\
32018 \cdot 2 \\
3202 \cdot 7 \\
3200 \cdot 5 \\
3200 \cdot 1 \\
31056 \cdot 7 \\
31056 \cdot 0 \\
31055 \cdot 0 \\
31053 \cdot 7 \\
31050 \cdot 9 \\
31047 \cdot 9 \\
31047 \cdot 8\end{array}$ & $\begin{array}{l}29056 \cdot 9 \\
29057 \cdot 0 \\
29057 \cdot 2 \\
29057 \cdot 2 \\
29056 \cdot 7 \\
29056 \cdot 4 \\
29056 \cdot 4 \\
29056 \cdot 1 \\
29055 \cdot 6 \\
29053 \cdot 0 \\
29051 \cdot 9 \\
29051 \cdot 6 \\
29051 \cdot 3 \\
29051 \cdot 1 \\
29050 \cdot 9 \\
29050 \cdot 0 \\
29050 \cdot 2 \\
29050 \cdot 5 \\
29051 \cdot 9 \\
29052 \cdot 1 \\
29052 \cdot 1 \\
29052 \cdot 8 \\
29053 \cdot 3 \\
29053 \cdot 2 \\
29053 \cdot 0 \\
29052 \cdot 3 \\
29051 \cdot 7 \\
29051 \cdot 2 \\
29051 \cdot 1 \\
29051 \cdot 2 \\
29055 \cdot 4 \\
3002 \cdot 6 \\
3001.4 \\
3001.0 \\
29050 \cdot 3 \\
29057 \cdot 4 \\
29057 \cdot 1 \\
29056 \cdot 6 \\
29055 \cdot 2 \\
29054 \cdot 3 \\
29054 \cdot 2\end{array}$ & $\begin{array}{r}1.36 \\
0.98 \\
3.82 \\
2.18 \\
3.17 \\
5.46 \\
0.99 \\
17.07 \\
10.11 \\
8.92 \\
7.40 \\
3.64 \\
15.54 \\
11.77 \\
2.18 \\
27.12 \\
2.72 \\
2.35 \\
11.76 \\
4.44 \\
1.40 \\
3.13 \\
2.31 \\
2.73 \\
3.63 \\
2.45 \\
10.16 \\
2.78 \\
7.36 \\
2.48 \\
17.20 \\
15.27 \\
2.29 \\
0.53 \\
4.07 \\
1.08 \\
0.87 \\
1.23 \\
2.86 \\
2.69 \\
0.19\end{array}$ & 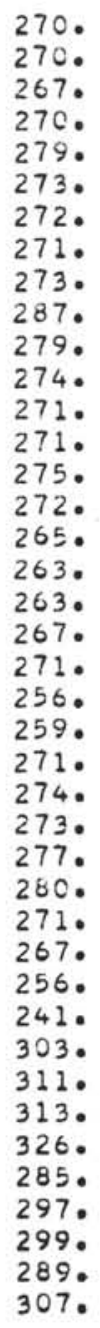 & $\begin{array}{l}7.434 \\
5.935 \\
6.957 \\
6.907 \\
60803 \\
3.722 \\
3.718 \\
8.986 \\
6.193 \\
5.250 \\
7.403 \\
7.293 \\
7.285 \\
5.695 \\
5.968 \\
6.212 \\
5.844 \\
5.886 \\
6.659 \\
6.503 \\
6.466 \\
5.886 \\
5.781 \\
6.848 \\
6.808 \\
5.890 \\
5.810 \\
50568 \\
5.668 \\
5.518 \\
5.702 \\
6.837 \\
5.109 \\
5.310 \\
5.682 \\
2.717 \\
40358 \\
30379 \\
3.584 \\
4.248 \\
2.965\end{array}$ & $\begin{array}{r}1.36 \\
2.35 \\
6.17 \\
8.36 \\
11.54 \\
17.00 \\
17.99 \\
35.06 \\
45.18 \\
54.10 \\
61.51 \\
65.15 \\
80.70 \\
92.47 \\
94.66 \\
121.78 \\
124.51 \\
126.87 \\
138.63 \\
143.07 \\
144.48 \\
147.62 \\
149.93 \\
152.67 \\
156.30 \\
158.75 \\
168.92 \\
171.71 \\
179.07 \\
181.56 \\
198.76 \\
214.03 \\
216.33 \\
216.86 \\
220.93 \\
222.002 \\
222.89 \\
224.13 \\
227.00 \\
229.69 \\
229.89\end{array}$ \\
\hline
\end{tabular}

